\title{
AGE DIFFERENCES IN COGNITIVE CONTROL OF EMOTIONAL MEMORY: METACOGNITIVE ASPECTS AND NEURAL CORRELATES \\ by
}

\author{
Sara Nicole Gallant
}

M.A., Psychology, Ryerson University, 2013

B.A. (Honours), Psychology, University of Windsor, 2011

\author{
A dissertation \\ presented to Ryerson University \\ in partial fulfillment of the \\ requirements for the degree of \\ Doctor of Philosophy \\ in the Program of \\ Psychology \\ Toronto, Ontario, Canada 2017 \\ (C)Sara Nicole Gallant 2017
}




\section{AUTHOR'S DECLARATION}

I hereby declare that I am the sole author of this dissertation. This is a true copy of the dissertation, including any required final revisions, as accepted by my examiners.

I authorize Ryerson University to lend this dissertation to other institutions or individuals for the purpose of scholarly research.

I further authorize Ryerson University to reproduce this dissertation by photocopying or by other means, in total or in part, at the request of other institutions or individuals for the purpose of scholarly research.

I understand that my dissertation may be made electronically available to the public. 


\title{
Age Differences in Cognitive Control of Emotional Memory: \\ Metacognitive Aspects and Neural Correlates
}

\author{
Doctor of Philosophy, 2017 \\ Sara Nicole Gallant \\ Psychology, Ryerson University
}

\begin{abstract}
Previous research has identified a divergent trajectory for the aging brain, characterized by declines in cognition and preservation in emotional processing. According to the socioemotional selectivity theory, reduced time horizons in later life cause a motivational shift to prioritize emotional goals, such as positive well-being. In service of these goals, older adults devote more cognitive effort to attend to and remember positive information; however, whether they can exert control over such information once it enters memory is not well understood. The primary aim of this dissertation was thus to examine age-related changes in cognitive control of emotional memory including its underlying metacognitive and neural components. In three experiments, young and older adults completed a cue- or value-based version of the item-directed forgetting task for positive, negative, and neutral words. Results consistently demonstrated that young and older adults could strategically control encoding of emotional information, by prioritizing relevant over irrelevant words in memory. This was evident when encoding was directed by tobe-remembered (TBR) or to-be-forgotten (TBF) cues as well as by numeric points that signaled a gain or loss of value (+10 vs. -10). Extending previous research on metacognition and aging, results indicated age invariance in prospective judgments of learning made during the encoding of TBR and TBF words that varied in emotion. In contrast, age groups differed when retrospectively monitoring the source of words. Whereas young adults' source monitoring was
\end{abstract}


not influenced by emotion or cues, older adults tended to attribute positive items to sources that were higher in value for memory (TBR or +10 cues), consistent with an age-related bias to prioritize positivity. Finally, age differences in event-related potentials underlying encoding of TBR and TBF words provided evidence that older adults may recruit additional resources in frontal regions of the brain to facilitate task performance. Moreover, in line with behavioural results, the ERP signatures of directed forgetting were not modulated by emotion. Altogether, this dissertation demonstrates that cognitive control over emotional memory is intact in later life; however, it highlights important age differences in the metacognitive and neural correlates of this ability. 


\section{Acknowledgements}

This dissertation is the culmination of four years of work, which I can only describe as a roller coaster of sorts: filled with anxiety at times and thrills at others. Perhaps one of the most important things I will take away from this experience is that learning is a journey enriched by the perspectives of others. As I close this significant chapter of my life, I would like to thank the many individuals that enriched and shaped my learning along the way.

I am grateful to have worked with my advisor, Dr. Lixia Yang, during my M.A. and Ph.D. at Ryerson University. Lixia taught me much about cognitive aging and consistently encouraged me to pursue my research ideas in this field; my dissertation would not have been possible without her guidance. I am also very fortunate to have worked with Dr. Julia Spaniol, whom I viewed as a mentor in many capacities during graduate school. Julia's passion for science has been not only inspiring but has challenged my way of thinking as a researcher. I'm incredibly thankful for the time and advice that she has provided along the way. I owe many thanks to Dr. Ben Dyson for sharing with me his enthusiasm and knowledge of the event-related potential (ERP) technique. Ben's training and our subsequent collaborations provided a foundation of knowledge for completing the ERP study described in this dissertation. I must also acknowledge Carson Pun for his integral role in this ERP study. I am appreciative of the generous technical support he provided when programming the ERP experiment as well as

during signal processing. Thank you as well to my dissertation examining committee: Drs. Colin MacLeod, Todd Girard, and Stéphanie Walsh-Matthews whose insightful comments and critiques helped to improve my dissertation.

Thank you to Cognitive Aging Lab (CAL) members, past and present, for providing a truly collegial environment to work in. I am particularly grateful to have gone through this 
journey from start to finish with Brenda Wong. Brenda has been a source of great support throughout graduate school and has consistently been available to discuss research ideas, offer advice, or to just talk about life beyond school. Her friendship has made this entire process more enjoyable than I ever expected. These projects would also not have been possible without the CAL research assistants that helped with data collection as well as the many young and older adults who participated in my experiments. I've had the pleasure of meeting older adult participants from various walks of life, which has enriched my understanding of the aging process beyond that of any research article.

Finally, I thank my friends and family that kept my life balanced throughout graduate school. Thank you to Michael for his reminders to never doubt myself. His belief in my ability to pursue this goal has always kept me motivated. Lastly, I am thankful for my parent's unwavering patience and support as I completed my education. From the beginning of my undergraduate degree, through my Ph.D., and now as I embark on the next transition in my career, they have consistently stood by my side. It is a luxury to have supportive colleagues, friends, and family like this—-these words simply cannot express my gratitude.

This research was supported by a Discovery Grant from the Natural Sciences and Engineering Research Council (NSERC) of Canada (to Dr. Lixia Yang), an NSERC Doctoral Scholarship (to Sara Gallant), and a Student Research Grant from the Psychology Foundation of Canada (to Sara Gallant). 


\section{Table of Contents}

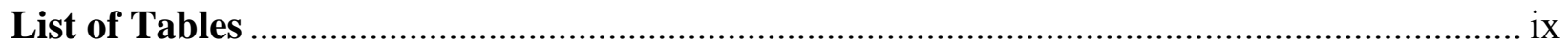

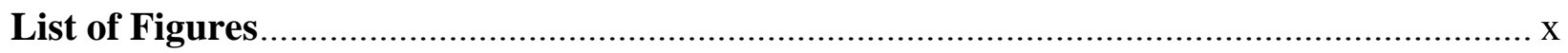

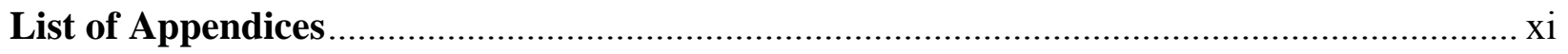

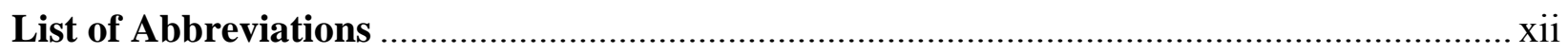

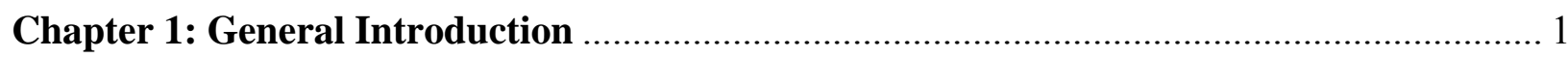

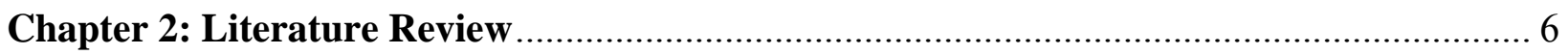

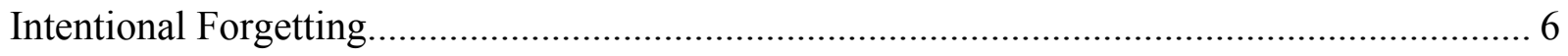

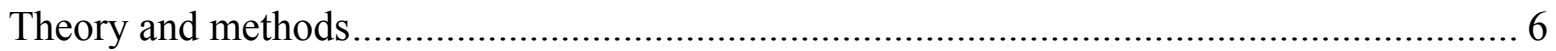

Cognitive and neural mechanisms ................................................................................... 10

Metacognition of intentional forgetting ........................................................................... 13

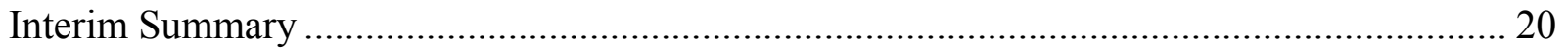

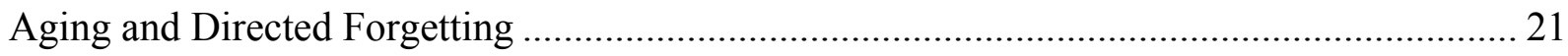

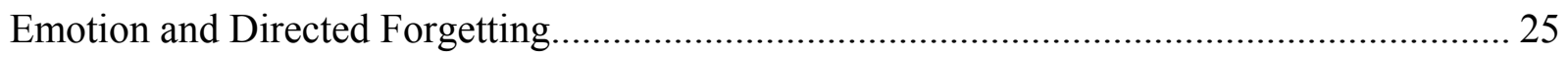

Aging-Emotion Interactions in Directed Forgetting ............................................................ 28

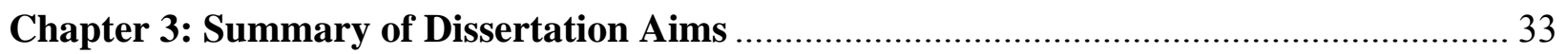

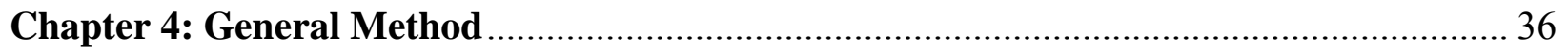

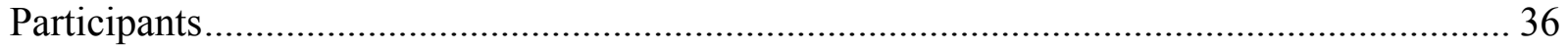

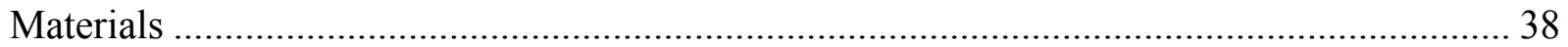

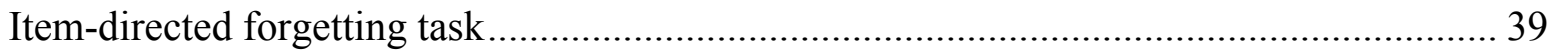

Cognitive, emotional, and demographic assessments......................................................... 40

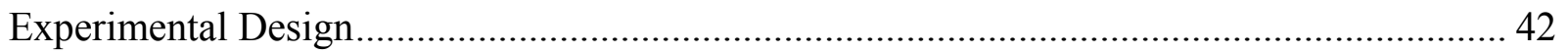

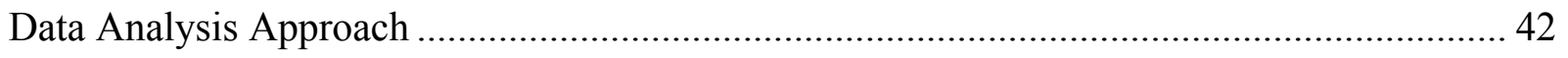

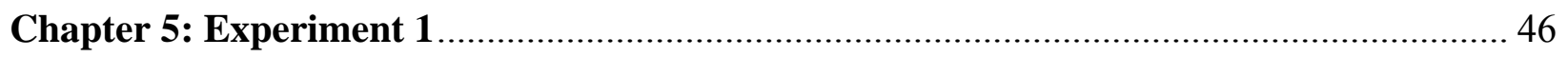

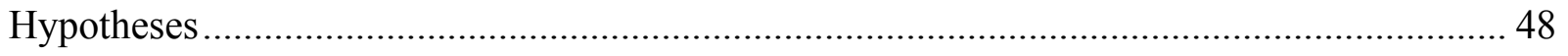


Method

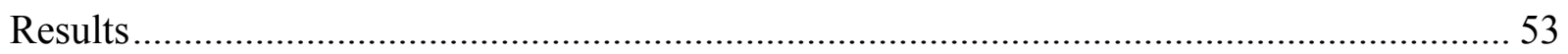

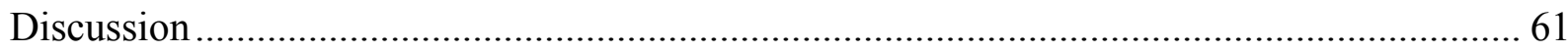

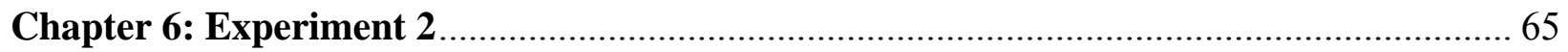

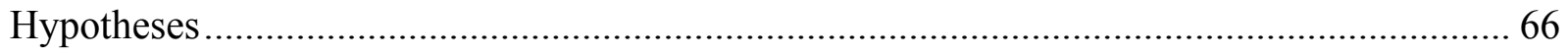

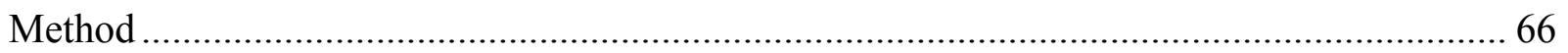

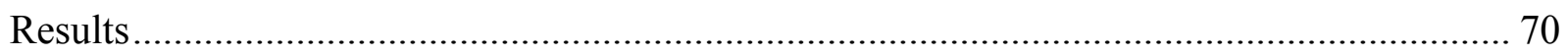

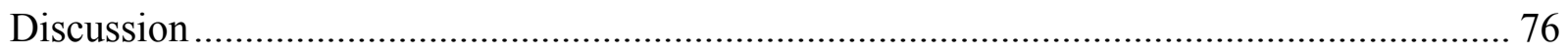

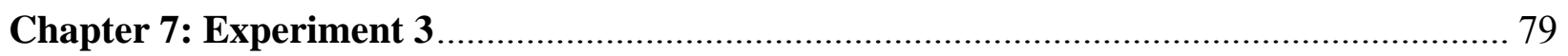

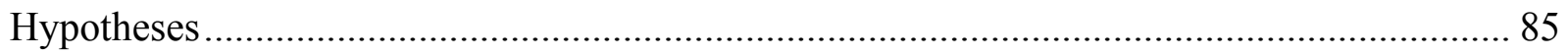

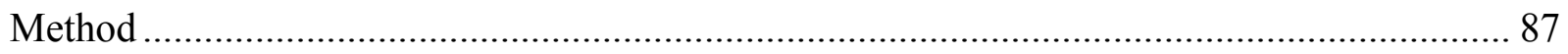

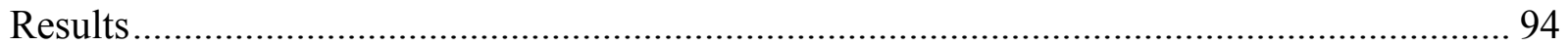

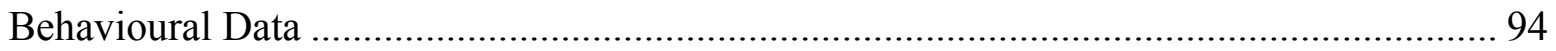

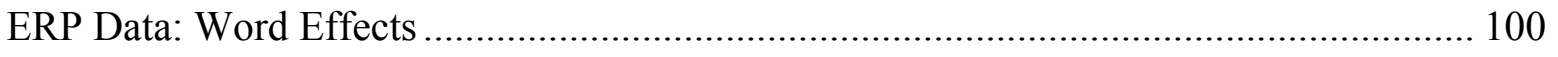

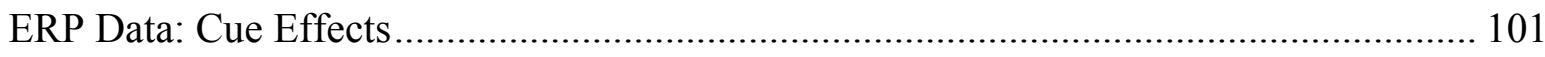

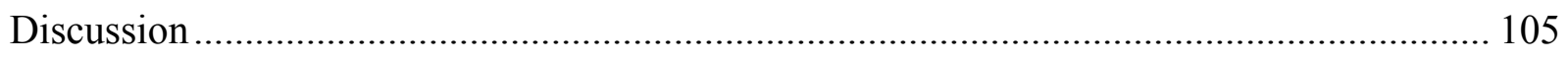

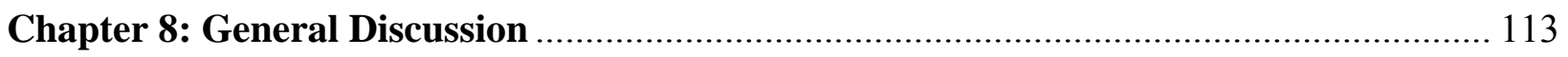

Cognitive Control of Emotional and Non-Emotional Memories...................................... 114

Metacognitive Aspects of Intentional Forgetting of Emotional Information ....................... 120

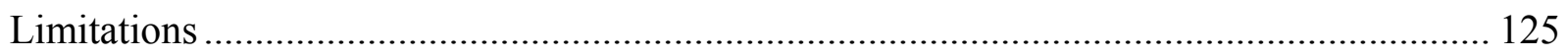

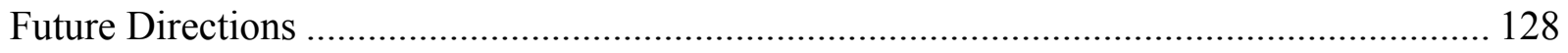

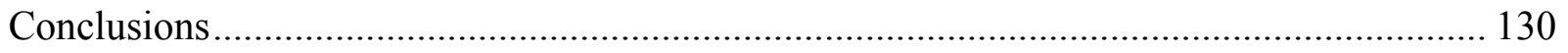

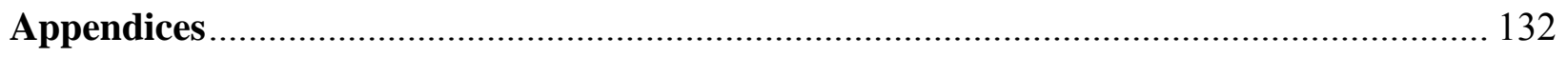

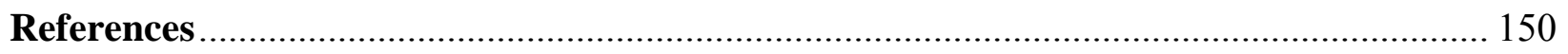




\section{List of Tables}

Table 1. Characteristics of the Stimuli used in Experiments 1-3 .......................................... 38

Table 2. Characteristics of the Final Sample in Experiment 1 ............................................ 50

Table 3. Means and Standard Deviations for Item Recognition Indices in Experiment 1........... 55

Table 4. Means and Standard Deviations for Source Monitoring Indices in Experiment 1 ........ 57

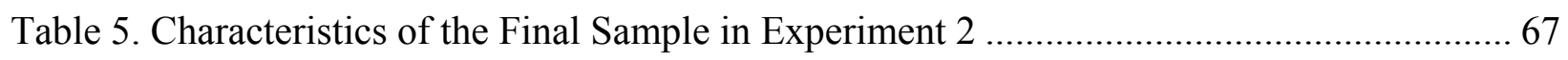

Table 6. Means and Standard Deviations for Item Recognition Indices in Experiment 2.......... 70

Table 7. Means and Standard Deviations for Source Monitoring Indices in Experiment 2 ........ 72

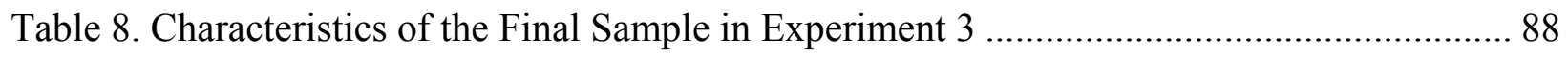

Table 9. Means and Standard Deviations for Item Recognition Indices in Experiment 3........... 95

Table 10. Means and Standard Deviations for Source Monitoring Indices in Experiment 3 ...... 98 


\section{List of Figures}

Figure 1. Directed forgetting procedures ....................................................................... 8

Figure 2. Sample TBF trial from the encoding phase of Experiment 1 ................................ 52

Figure 3. Mean JOL responses in Experiment 1 f................................................................ 54

Figure 4. Proportions of hits and false alarms in Experiment 1 ........................................ 56

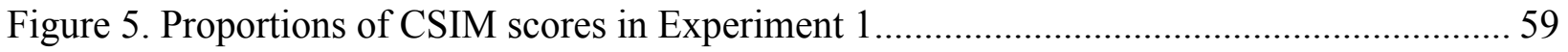

Figure 6. Proportions of misattributions of new items to sources in Experiment 1.................. 60

Figure 7. Sample of a high-value trial from the encoding phase of Experiment 2 ................... 69

Figure 8. Proportions of hits and false alarms in Experiment 2 ......................................... 71

Figure 9. Proportions of CSIM scores in Experiment 2 ....................................................... 74

Figure 10. Proportions of misattributions of new items to sources in Experiment 2................ 75

Figure 11. Layout of the 64-Channel International 10/20 system for recording EEG activity .... 89

Figure 12. Proportions of hits and false alarms in Experiment 3 ....................................... 96

Figure 13. Proportions of CSIM scores in Experiment 3.................................................. 99

Figure 14. Grand averaged ERPs from P1, Pz, and P2, time-locked to word onset................. 101

Figure 15. Grand averaged ERPs from P1, Pz, and P2, time-locked to cue onset.................... 102

Figure 16. Grand averaged ERPs from AF3, AFz, and AF4, time-locked to cue onset............ 104 


\section{List of Appendices}

Appendix I: Consent Form for Older Adults in Experiment 1 ........................................... 132

Appendix II: Consent Form for Young Adults in Experiment 1 ........................................... 134

Appendix III: Debriefing form for Experiment 1 ............................................................. 136

Appendix IV: Consent Form for Older Adults in Experiment 2 ........................................ 137

Appendix V: Consent Form for Young Adults in Experiment 2 ....................................... 139

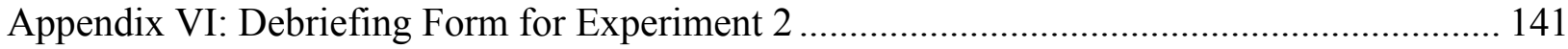

Appendix VII: Consent Form for Older Adults in Experiment 3 ....................................... 142

Appendix VIII: Consent Form for Young Adults in Experiment 3 ...................................... 144

Appendix IX: Debriefing Form for Experiment 3 ........................................................... 146

Appendix X: Stimuli selected for Experiments 1-3 .......................................................... 147

Appendix XI: MATLAB script used for signal processing and ICA ................................... 148 


\section{List of Abbreviations}

ANEW

ANOVA

CSIM

DASS-21

DSST

EEG

EOG

ERP

fMRI

HPC

ICA

ISI

JOL

LPP

MTL

PANAS

PFC

SBT

TBR

TBF
Affective Norms for English Words

Analysis of variance

Conditional Source Identification Measure

21-item Depression Anxiety and Stress Scale

Digit Symbol Substitution Test

Electroencephalography

Electrooculogram

Event-related potential

Functional magnetic resonance imaging

Hippocampus

Independent components analysis

Inter-stimulus interval

Judgment of learning

Late positive potential

Medial temporal lobe

Positive and Negative Affect Schedule

Prefrontal cortex

Short Blessed Test

To-be-remembered

To-be-forgotten 


\section{Chapter 1: General Introduction}

Memory encompasses processes that encode, manipulate, and store information for later reconstruction. According to models of human memory, working memory acts as a temporary workspace where incoming information is manipulated and temporarily stored (Baddeley \& Hitch, 1974), some of which becomes encoded for long-term retrieval (Atkinson \& Shriffin, 1968). These vital processes in turn allow us to learn new information, make informed decisions, and make sense of the world around us. Although much of psychological research on memory focuses on successful remembering, the ability to forget is just as critical to this system. Just like remembering, forgetting is not a unitary process. While some information may be incidentally lost as a result of poor encoding or transience (Schacter, 1999), intentional forgetting benefits memory by reducing clutter from no-longer-relevant information. For instance, if a family physician reduces the dosage of a prescription to regulate blood pressure, it becomes necessary to update memory and store the new dosage while forgetting the former. In scenarios such as this, discarding the unwanted information (the former prescription) reduces interference in memory by clearing a path for more relevant information (the new prescription). This form of goal-directed forgetting thus represents a process by which we strategically control information in memory to optimize successful remembering of relevant details while ridding memory of irrelevance.

One of the most fascinating aspects of the memory system is its malleability. It is well documented that factors like emotion can strengthen memory traces, whereas inevitable processes like healthy aging can weaken memory abilities. This latter finding is evidenced not only by an increase in self-reported memory complaints with age (e.g., Gilewski, Zelinski, \& Schaie, 1990; Reid \& MacLullich, 2006) but also by decades of empirical research documenting 
age-related declines in episodic memory (for a review, see Grady \& Craik, 2000). Typically, these experiments show that, relative to their young counterparts, older adults have trouble with the conscious recollection of episodic information from memory (Grady, 2012). This, in turn, translates to reduced memory for the content of an episodic event (i.e., item memory) as well as for more specific contextual details such as when or where it occurred (i.e., source memory; Craik \& Byrd, 1982; Johnson, Hashtroudi, \& Lindsay, 1993; Old \& Naveh-Benjamin, 2008; Spaniol, 2015; Spencer \& Raz, 1995). Given such declines in episodic memory and its underlying cognitive processes, we might also assume that aging is accompanied by increased forgetting. While this may be true for incidental forgetting (Maylor, 1993), the ability to engage in the controlled, intentional form of forgetting has also been found to diminish in later life (for a meta-analytic review, see Titz \& Verhaeghen, 2010). Such declines are alarming as they can have ramifications for memory performance, most notably, increased interference from irrelevant representations (Zacks, Radvanksy, \& Hasher, 1996). Various theoretical explanations have been offered to account for these memory deficits in aging, including reductions in the speed at which relevant cognitive operations can be executed (Salthouse, 1996), reduced inhibition of irrelevant or interfering information (Hasher \& Zacks, 1988; Lustig, Hasher, \& Zacks, 2007), and declines in the ability to bind the constituent elements of a memory together (Naveh-Benjamin, 2000; Old \& Naveh-Benjamin, 2008).

Although this research paints a "doom and gloom" picture of aging, it is important to note that some processes remain stable or even improve as we get older. Whereas age-related declines in "cold" cognitive processes are well documented, preservation has been observed in the "hot" emotional functions of the aging brain (for reviews, see Carstensen, Mikels, \& Mather, 2006; Mather, 2012; Mather \& Carstensen, 2005; Scheibe \& Carstensen, 2010). This research has 
shown that, relative to young adults, older adults are more likely to report increased positive well-being (Carstensen et al., 2010), show enhanced emotional problem solving (BlanchardFields, 2007), and demonstrate a better ability to regulate or "bounce back" from negative moods (Ebner \& Fischer, 2014). According to a 10-year longitudinal examination, such improvements in emotional functioning, including the ratio of positive to negative emotions, and stability in day-to-day emotional experience, seem to begin early in adulthood and steadily increase with age (Carstensen et al., 2010).

Perhaps more intriguing is research concerning how the "hot" emotional and "cold" cognitive processes of the brain interact in aging. Indeed, older adults' stable emotional processes seem to have far-reaching effects on how information is processed and stored in memory (Mather, 2010). Research in this field led to the finding of an age-related positivity effect, a phenomenon characterized by a relative preference for positive over negative information in older adults' information processing (for a review, see Reed \& Carstensen, 2012). For example, when given the option to attend to a pair of positive or neutral facial expressions, older relative to young adults show a preference to fixate on faces with a positive expression (e.g., happy). However, when shown a sad-neutral expression pair, older adults tend to ignore or look away from the negative expression more than do young adults (Isaacowitz, Wadlinger, Goren, \& Wilson, 2006; Mather \& Carstensen, 2003). Unsurprisingly, this attentional preference for positive over negative materials can also influence older adults' memory performance. For instance, when compared to middle-aged or young adults, older adults' long-term memory has been shown to be elevated for positive over negative images, whereas memory for negative material declines linearly with age (Charles, Mather, \& Carstensen, 2003). Since these seminal investigations, the age-related positivity effect has been extended to other measures of memory, 
including autobiographical memory and working memory (e.g., Kensinger, 2008; Mather \& Carstensen, 2005; Reed, Chan, \& Mikels, 2014).

Older adults' preserved emotional processes and the age-related positivity effect can be fit within motivational theories of aging, which propose that changes in intrinsic motivation cause older adults to become selective in where they invest their cognitive resources (Hess, 2014). According to socioemotional selectivity theory, older adults' time left in life is perceived as limited relative to young adults whose time horizons are perceived as expansive (Carstensen, 1995). Under such time constraints, motivations and priorities change with age, with a greater emphasis placed on goals that are present-focused and emotionally meaningful (Charles \& Carstensen, 2009). These goals, in turn, influence what we choose to pay attention to and remember in our environment. As a result, older adults show a bias toward positive stimuli in service of emotionally-relevant goals such as positive well-being (e.g, Carstensen \& Mikels, 2005; Isaacowitz et al., 2006; Kensinger \& Corkin, 2003; Kensinger, 2008; Mather \& Carstensen, 2003). Young adults, on the other hand, do not show this preference, as their goals are less concerned with emotion and typically are oriented toward the future (e.g., knowledge acquisition in pursuit of a degree or career). Instead, they often show a negativity bias or general bias toward emotional stimuli (e.g., Charles et al., 2003; Kensinger, 2008).

Whereas the socioemotional selectivity theory proposes that the positivity effect is driven by goal-directed behaviour, other theories have attributed the effect to declining cognitive functions. For example, the dynamic integration theory contends that older adults increase their focus on positive information because it is less complex and requires fewer cognitive resources to process than negative information (Labouvie-Vief, 2003). This explanation is thus rooted in automatic processes that act in response to changes in cognitive ability as opposed to goal- 
directed processes that modulate behaviour. However, empirical evidence seems to favour the top-down explanation of the age-related positivity effect given that it is typically not observed in older adults with reduced cognitive control (Mather \& Knight, 2005) or when cognitive resources are constrained by task demands (e.g., by dividing attention; Reed et al., 2014). Findings such as these support the strategic nature of the positivity effect in older adults' memory and challenge views that attribute this effect to cognitive decline.

\section{Broad Overview of the Dissertation}

Although there is quite a bit of research surrounding how older adults actively prioritize positive information in attention and memory (Reed et al., 2014), less research has concerned how this positivity bias might influence the ability to exert control over information once it has entered memory. The overarching goal of this dissertation is to shed light on this topic by examining age differences in the ability to intentionally forget emotional information. In addition, it will examine the metacognitive aspects and neural correlates underlying these processes. The purpose of the following chapter is to orient the reader to the literature most relevant to this dissertation. First, I will introduce the concept of intentional forgetting, including theory, methods, as well as the proposed underlying neural and metacognitive mechanisms. Once this foundational literature has been covered, I will detail the existing research examining the impact of emotion and aging on intentional forgetting as well as some of my own work on the interaction of these factors. Taken together, the goal of Chapter 2 is not only to provide an understanding of the literature but also to build a rationale for the set of experiments presented in the ensuing chapters. 


\section{Chapter 2: Literature Review}

\section{Intentional Forgetting}

For most of us, forgetting is perceived as one of the "sins" of memory that we wish to avoid (Schacter, 1999). It is associated with lost wallets, forgotten names, or missed appointments. As we age, forgetting is feared. It means a "senior's moment" or worse, an indicator of dementia. This narrow-minded view, however, fails to consider the necessity of forgetting for optimal memory and even emotional functioning. Consider a painful experience that we wish to suppress, such as the death of a loved one or the terrorist attacks of 9/11. The inability to control such memories can be debilitating and may result in intrusive reminders of the traumatic event - a hallmark feature of clinical diagnoses such as posttraumatic stress disorder (PTSD; American Psychiatric Association, 2013). Although this may be an extreme example, it illustrates just how crucial forgetting is to daily functioning. When intentional, it allows us to control or "clean up" the contents of memory, reducing interference from unwanted information. In recent decades, memory researchers have been trying to elucidate the workings of intentional forgetting processes, which has resulted in the development of various methods for manipulating forgetting and a vast literature on its corresponding mechanisms. The following section provides an overview of such theory and methods.

Theory and methods. The literature on intentional forgetting provides a foundation for understanding how we control information once it has entered memory. Different from the common "passive decay" conception, intentional forgetting can be viewed as an active process in which to-be-forgotten (TBF) items are suppressed from further processing or retrieval and to-beremembered (TBR) items are selectively rehearsed. A few ways to induce intentional forgetting have been outlined in the literature. Early work by Muther (1965) employed a simple cueing 
paradigm in which half of the items on a study list were either preceded or followed by a cue deeming the item as irrelevant. Results demonstrated that participants could exclude these irrelevant items from short-term memory storage. In addition, recall of TBR items in the cued condition was greater than when participants studied the same number of words but without cues to forget, implying that cues benefited memory for TBR items. Later work by Bjork, LaBerge, and Legrand (1968) provided further corroborating evidence that a drop cue to forget was advantageous to memory. In their paradigm, one condition of participants studied a list of two consonant strings intermixed among a series of digits. At the end of list, participants recalled the consonant strings, beginning with the second string. In another condition, participants studied the same list of digits and consonant strings, except that an instruction to forget was provided after the first string was presented. At the end, participants again recalled the second string. Relative to the first condition, the cued group showed better memory for the second string. From these findings, Bjork and colleagues argued that the drop instruction improved participants' performance by reducing proactive interference from the first item in memory. This work, along with other seminal studies (e.g., Bjork, 1970), led to the view that forgetting, when intentional, is positive as it prevents TBF information from interfering with the processing of more relevant details (Bjork, 1972; Bjork, Bjork, \& Anderson, 1998). As suggested by Bjork (1972, p. 218), without such processes, "we would degenerate to a proactive-interference-induced state of total confusion".

When studying intentional forgetting in the lab, one of the most often used procedures has been the directed forgetting paradigm (for a review, see MacLeod, 1998). Two versions of the task exist that differ primarily in the timing and frequency of cue presentations (Basden \& Basden, 1998). In the item method, cues are presented on an item-by-item basis (e.g., Gallant \& 
Yang, 2014; Gallant \& Dyson, 2016; Thompson, Fawcett, \& Taylor, 2011). Most often, the cue is presented following each item to ensure that all stimuli are studied and encoded to the same extent (see Figure 1A), although there are some occasions where cues are presented simultaneously with the item (e.g., Bailey \& Chapman, 2012) or before (e.g., 'pre-cue' condition; Muther, 1965). A within-subject comparison of memory for TBR versus TBF information reveals the directed forgetting effect, characterized by higher memory for TBR relative to TBF items. The larger this difference, the greater the effect. In the list method, two groups of participants study two lists of stimuli (e.g., MacLeod, 1999; Minnema \& Knowlton, 1998; Sego, Golding, \& Gottlob, 2006). Following the first list, one group receives a cue to forget while another group receives a cue to remember. Both groups then study a second list, which is TBR for both groups (Figure 1B). Different from the item method, a between-subjects comparison reveals the directed forgetting effect in the list method: Relative to the remember-all group, the forget-cued participants show reduced recall of TBF items from the first list (the cost of directed forgetting) and a tendency to recall more TBR items from the second list (the benefit of directed forgetting).

A) Item-Directed Forgetting

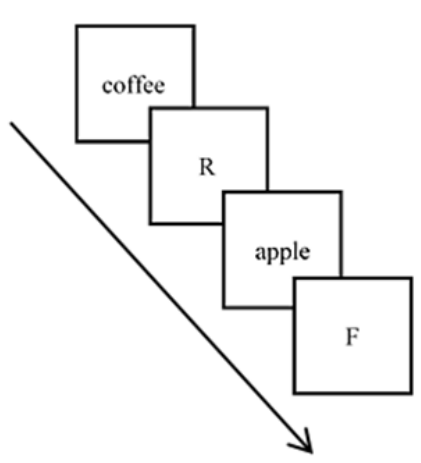

B) List Directed Forgetting

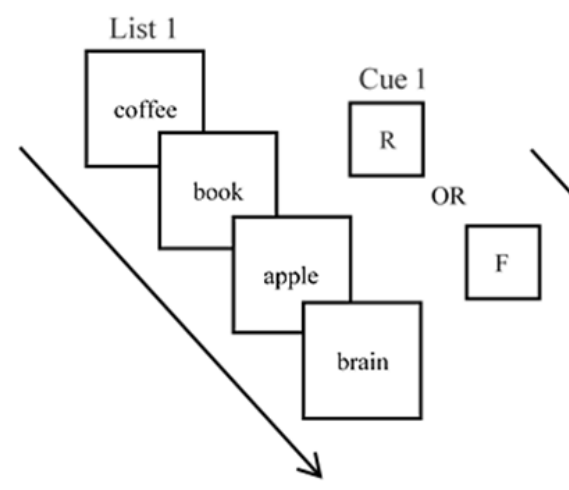

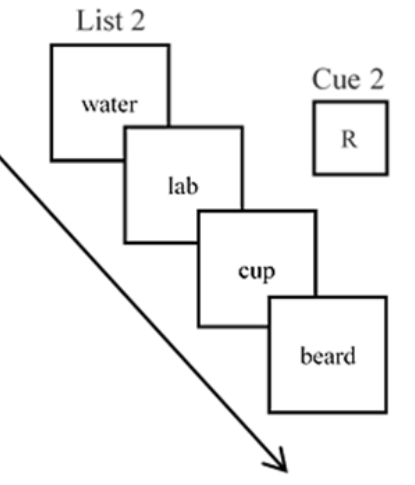

Figure 1. Directed forgetting procedures: A) Item-directed forgetting task: cues are delivered on an item-by-item basis; B) List directed forgetting task: cues are delivered on a list-wise basis. $\mathrm{R}=$ Remember, $\mathrm{F}=$ Forget. 
Another method that has had a great impact on the intentional forgetting field was developed by Anderson and Green (2001) and referred to as the think/no-think procedure. In this thought-suppression paradigm, participants first study a series of cue-target pairs (e.g., pictures or word pairs) and learn to recall the target upon presentation of the cue (i.e., the study phase). For example, they might see the cue-target pair "brain-apple" and they will need to learn the pair until they can recall "apple" when prompted with the word "brain". After each pair has been learned to a certain criterion, each cue is presented individually and participants are instructed to either think or not think about the target associated with that cue (i.e., think/no-think phase). To perform the task successfully during this phase, participants must retrieve the target items on think trials and suppress them on no-think trials. During a subsequent test phase, each cue is shown again and participants recall the associated target. The resulting think/no-think effect is characterized by lower memory for no-think items relative to both think and baseline items that were excluded from the think/no-think phase (for a review, see Anderson and Hanslmayr, 2014). Such an effect has been theorized to indicate that no-think items have been successfully suppressed.

Although each of these methods offers a way to induce forgetting via explicit cue manipulations to forget items or suppress thoughts, they are not necessarily driven by similar processes (e.g., Basden \& Basden, 1996). For example, some research has shown that, under the item method, the directed forgetting effect can be observed on both recall and recognition tasks, whereas only recall tasks produce the directed forgetting effect with the list method (e.g., Basden, Basden \& Gargano, 1993). Such findings have led to the idea that these procedures may rely on divergent mechanisms. Thus, intentional forgetting is not a uniform process, but rather as described by MacLeod (1998) and Bjork (1998) - represents a multifaceted 'set of tools' for 
studying forgetting in human memory. The following section provides an overview of the mechanisms underlying intentional forgetting.

Cognitive and neural mechanisms. Several theories have attempted to account for intentional forgetting effects, with different mechanisms proposed to underlie each method. The think/no-think task and list method directed forgetting are thought to reflect mechanisms operating at retrieval that reduce the accessibility of TBF or no-think items in memory (for a review, see Anderson \& Hanslmayr, 2014; Basden \& Basden, 1998). This is because, under these methods, items are fully encoded prior to a no-think or forget instruction. For instance, during a no-think trial, participants are theorized to stop retrieval of these items using inhibitory mechanisms like those used to stop a reflexive motor response (Anderson, 2005; Anderson \& Green, 2001; Anderson \& Levy, 2009). In the list method, the retrieval inhibition account proposes that an inhibitory mechanism blocks access to the TBF items during retrieval. Support for this account has come from studies showing that the directed forgetting effect of the listmethod can be wiped out when TBF items are presented again during a recognition test, resulting in a "release from inhibition" (Basden et al., 1993; but also see Sahakyan, Waldum, Benjamin, \& Bickett, 2009). In addition to retrieval based accounts, theories of selective rehearsal have been proposed to account for directed forgetting effects under the list-method (Sheard \& MacLeod, 2005). According to this view, items are segregated and organized into their respective cue-based categories during encoding after which only TBR items are selectively rehearsed and thus better remembered than TBF items.

The item-method of directed forgetting has usually been attributed to encoding-based processes such as selective rehearsal of TBR relative to TBF items (Anderson \& Hanslmayr, 2014; Basden \& Basden, 1996). Advocates for this hypothesis argue that each item is held in 
working memory until a cue is presented. If the cue signals "remember", the participant engages in elaborative rehearsal to commit the item to memory; if the cue signals "forget", the item is dropped from rehearsal processes and passively decays. Support for such views come from findings that recollection of contextual details is higher for items receiving a TBR cue relative to a TBF cue, suggesting that these items received greater distinctive processing during encoding (e.g., Basden \& Basden, 1996, Experiment 3; Gardiner, Gawlik, \& Richardson-Klavehn, 1994). Other researchers (e.g., Hauswald, Schulz, Iordanov, \& Kissler, 2010; Zacks et al., 1996) suggest that item-method directed forgetting involves inhibitory mechanisms that operate during encoding, such as attentional inhibition. Different from the selective rehearsal view, the inhibitory account proposes that an intentional withdrawal of attention reduces processing of TBF items, freeing up resources for the encoding of TBR items.

Several behavioural studies have attempted to test the inhibitory account of directed forgetting such as Zacks and colleagues (1996), who compared young and older adults in itemdirected forgetting efficiency (Experiments 1A and 1B). Using their inhibitory deficit hypothesis of aging (Hasher \& Zacks, 1988) as theoretical motivation, the authors hypothesized that older adults should be less successful in implementing cues to forget, which is exactly what they found. Relative to young adults, older adults were less efficient at eliminating TBF items from memory, were slower to reject TBF items on recognition tests, and exhibited greater intrusions from TBF items in their recall. The authors attributed these results to age-related declines in attentional inhibition, lending support to the inhibitory account of item-directed forgetting. Fawcett and Taylor (2008) further tested the inhibitory theory by investigating the attentional demands of directed forgetting using a dot-probe detection task following presentation of the memory cue. According to an active account of item-directed forgetting, if cues to forget evoke 
an inhibitory mechanism, the ability to carry out a secondary task following a TBF cue should be diminished relative to a TBR cue. Consistent with this, participants' in Fawcett and Taylor's study showed slower reaction times to detect dot-probes following a TBF cue relative to TBR cue, suggesting that intentional forgetting in the item method may operate through an active withdrawal of attention. This challenges the view that TBF items are selectively non-rehearsed. Linking behaviour to brain activity, neuroimaging research has shown that distinct brain regions contribute to intentional remembering and forgetting. Functional magnetic resonance imaging (fMRI) studies examining hemodynamic responses in the brain suggest that encodingrelated processes in the left inferior prefrontal cortex (PFC), medial temporal lobe (MTL), posterior cingulate cortex, and occipital cortex support successful remembering of TBR items (Bastin et al., 2012; Rizio \& Dennis, 2013, 2014; Wylie, Foxe, \& Taylor, 2008). In contrast, as reviewed by Anderson and Hanslmayr (2014), intentional forgetting is shown to activate the right superior PFC and middle frontal gyrus as well as the right inferior parietal lobe, which have been implicated in attentional control and inhibitory processing (e.g., Bastin et al., 2012; Rizio \& Dennis, 2013). Functional connectivity analyses examining relationships between distributed brain regions have also revealed a link between the dorso-lateral PFC (DLPFC) and the hippocampus (HPC) during successful intentional forgetting such that increases in DLPFC activity following TBF cues predicts decreases in left HPC activity in the MTL. This functional relationship may imply that inhibitory mechanisms associated with the DLPFC suppresses encoding-related activity in the MTL following a cue to forget (Anderson \& Hanslmayr, 2014; Rizio \& Dennis, 2013).

Studies using event-related potentials (ERP) derived from scalp-recorded electroencephalography (EEG) provide further support for the role of distinct brain mechanisms 
in the directed forgetting effect. For instance, Paz-Caballero, Menor, and Jiménez (2004) found that TBF cues evoked more positive-going ERP activity over frontal sites whereas TBR cues evoked more positive ERP amplitudes over parietal sites. These cue-related effects were also most prominent in participants classified as having a "high forgetting effect" via median split. Relative to high forgetters, participants in the "low forgetting effect" group showed a more distributed pattern of activity with greater ERP positivity over frontal as opposed to parietal sites following TBR cues. The authors thus proposed that successful directed forgetting involves the engagement of rehearsal mechanisms following TBR cues (parietal positivity) that keep the item on "standby" until the next stimulus, whereas TBF cues may elicit inhibitory mechanisms (frontal positivity) that prevents further processing of these items. ${ }^{1}$

In sum, these behavioural, fMRI, and ERP findings support the notion that directed forgetting is a goal-driven process that draws on multiple mechanisms to selectively rehearse TBR items and actively de-prioritize the processing of TBF items ${ }^{2}$.

Metacognition of intentional forgetting. Given the controlled nature of intentional forgetting, an interesting question that arises is whether participants are consciously aware of their ability to adapt performance to remember what is important and forget what is irrelevant as well as how these items become subsequently organized and experienced within memory. The

\footnotetext{
${ }^{1}$ Paz-Caballero and colleagues' (2004) study is only one of many ERP studies examining the neural correlates of directed forgetting. This literature will be further detailed in Chapter 7, in which Experiment 3 is described that used the ERP technique to examine age differences in the ERP correlates of directed forgetting for positive, negative, and neutral information.

${ }^{2}$ The item-method is of interest to the current dissertation as it more readily allows for examining emotional effects on intentional forgetting using mixed lists of positive, negative, and neutral stimuli. The use of mixed lists is important to the design of these experiments as it increases the distinctiveness of emotional stimuli against intermixed neutral items whereas pure lists are less likely to produce emotionally enhanced memory (Dewhurst \& Parry, 2000). When studying age and emotion interactions, the ability to make within-subject comparisons of emotional memory is also critical as the positivity effect is often characterized by greater memory for positive over negative items in older adults. The list method is thus not suitable for the current dissertation's goals as pure lists would be needed to disentangle the effects of emotion on directed forgetting given that cues are delivered at the end of each list (e.g., Minnema \& Knowlton, 2008). For this reason, the literature reviewed from this point forward will concern the item method only.
} 
literature on metacognition provides a foundation for understanding such cognitive awareness. Metacognition is often described as "cognition about cognition" and can be defined as knowledge or awareness of our own cognitive abilities (Flavell, 1979). In the context of memory, metacognition (often referred to as metamemory) encompasses the inferential processes that allow us to monitor the content of our memories and make judgments about our past, present, or future performance (Metcalfe \& Dunlosky, 2008). For example, we can monitor our ongoing study performance to judge the likelihood that we will later remember that information on a future test. In this way, monitoring becomes a crucial part of learning by allowing us to evaluate our memory and make decisions to flexibly adapt performance as needed. Consider a student studying for an exam: They will be better prepared if they are able to monitor what information has already been learned and use that knowledge to inform their decision to move on to new material (Koriat, 2007). In addition to monitoring learning performance, metacognition also allows us to evaluate our memory and make inferences about the origin of information by reflecting on the characteristics or features that were stored alongside the information (Kuhlmann \& Bayen, 2016; Mitchell, 2016). In the context of directed forgetting, research on its metacognitive aspects is limited, although a few studies can shed light on how we monitor and make judgments about TBR and TBF memories.

Judgments of learning (JOLs). One such line of research has examined how participants make prospective judgments about their ability to control encoding of TBR and TBF items via judgments of learning, one of the most frequently investigated forms of metacognitive monitoring (for reviews, see Hertzog \& Hultsch, 2000; Metcalfe \& Dunlosky, 2008). When making JOLs, participants monitor and evaluate their memory to determine the likelihood that they will remember a just-studied stimulus on a later memory test. As such, they are an effective 
means to measure an individual's predictive accuracy for the stimuli under test. Typically, a high JOL response reflects the belief that the stimulus has been learned and will be later remembered whereas a low JOL response reflects the belief that the item is not likely to be remembered. The relative accuracy of JOLs refers to how well one's judgments differentiate their performance on the task at hand and is typically gauged using the Goodman-Kruskal gamma correlation (Goodman \& Kruskal, 1954; Nelson \& Narens, 1990). Gamma is computed between JOLs and recognition at the item-level, with more positive correlations indicating better relative accuracy of JOLs. The averaged gamma across participants is then compared against zero to determine whether the relative accuracy for a given condition is above chance. If gamma significantly exceeds zero, we can say that relative accuracy is greater than chance. ${ }^{3}$

According to Koriat's (1997) cue-utilization theory, intrinsic and extrinsic cues can be used to guide JOLs during a memory task. Intrinsic cues encompass features of the to-be-learned stimulus, such as its ease of learning (e.g., degree of relatedness between two members of a paired associate). In contrast, extrinsic cues are characterized by the learning conditions (e.g., presentation time) or the cognitive operations applied by the learner during encoding (e.g., records of elaborative encoding processes). Directed forgetting memory cues (i.e., TBR vs. TBF) can thus be thought of as extrinsic, given the differences in cognitive operations that each of these cues evoke. The learner should thus attribute higher JOLs to TBR items that receive greater rehearsal and lower JOLs to TBF items that are suppressed. To determine whether this is indeed the case, Friedman and Castel (2011) administered an item-directed forgetting task, in which young adults provided JOLs following each TBR or TBF item. Results indicated that both recall

\footnotetext{
${ }^{3}$ The Goodman-Kruskal gamma statistic (Goodman \& Kruskal, 1954) is a nonparametric index of association often used to quantify the accuracy of JOLs for predicting memory performance within the metacognitive monitoring literature (Nelson \& Narens, 1990). The key assumption of the gamma statistic is that all data are ordinal; thus, when computing correlations, it is only the rank-ordering of judgment that matters, not the absolute magnitude. Gammas are thus calculated for each participant at the item-level between JOLs and recognition accuracy.
} 
and JOLs were higher for TBR than for TBF items, confirming that directed forgetting cues can be effectively utilized when monitoring learning performance with JOLs. Moreover, the authors found that participants' JOLs were reasonably calibrated with objective memory for TBR and TBF items, despite some overconfidence in their overall ability to recall each item type. Taken together, these findings imply that participants do have some metacognitive awareness of their ability to control memory.

As described in a recent review by Sahakyan and Foster (2015), having metacognitive awareness of our ability to intentionally forget is important to consider as such knowledge can contribute to how we allocate resources to forget irrelevant information. Specifically, before making the decision to intentionally forget, we need to first monitor how well that information has been learned. If we feel the information has not been committed to memory, we may be less likely to implement strategies to suppress the accessibility of that information. The ability to make these decisions thus requires us to monitor cues about the characteristics of information such as their memorability or how long we studied the information (Koriat, 1997) in order to determine how much effort we should exert. Sahakyan and Foster (2015) also argue that metacognitive beliefs may influence whether we adopt strategies to intentionally forget. For example, if we hold a belief that our memory is poor, as is often the case in later life, we may be less likely to engage strategies to intentionally forget. Thus, effective metacognitive monitoring of intentional forgetting is important to understand as it may influence how we choose to allocate cognitive resources when prioritizing TBR and suppressing TBF items.

Source monitoring. Other directed forgetting studies have examined how participants retrospectively make attributions about the origin of TBR and TBF stimuli in memory such as whether the item was initially encoded in the context of a TBR or TBF cue. The cognitive 
processes involved in making such judgments fall under the umbrella of source monitoring (Johnson et al., 1993). According to Mitchell (2016), source monitoring represents a quintessential aspect of metacognition as it comprises the mechanisms that encode and organize the content of memories as well as processes that evaluate and make judgments about retrieved information. These evaluative processes are what allow us to make inferences about the source or origin of our memories (Johnson et al., 1993). In daily life, we use source monitoring to keep track of where or who we learned information from, which can be important when inferring the credibility of a memory (e.g., "was it my friend or my doctor who suggested I stop taking my medication?") or monitoring internally versus externally generated information (e.g., "did that actually happen or did I only imagine it?"). Failure in such processes, as proposed by Johnson and colleagues (1993), underlies much of memory distortions or instances in which we attribute a memory to be something it is not.

The source monitoring framework put forward by Johnson and colleagues (1993) provides a foundation for understanding how we make such inferential decisions about the origins of our memories. A central tenet of this framework is that we generally do not retrieve source of information directly. Rather, we base our decisions on characteristics of the memory that are formed during encoding including contextual features (e.g., spatial location), emotional reactions, or cognitive operations. These characteristics are then bound together with the central elements of the memory (i.e., the item), distinguishing them from other representations. This facilitates our ability to later retrieve not just the item, but its characteristic features as well (Spaniol, 2015). Along with memory characteristics, Johnson and colleagues (1993) proposed that our source monitoring judgments can be influenced by pre-existing knowledge, beliefs, and current agendas or goals. For instance, when trying to remember the source of a memory, we 
may base our decisions on our beliefs or expectations about what a memory from a certain source ought to be like. This, in turn, can lead to reliance on stereotypes (Mather, Johnson, \& DeLeonardis, 1999) or schematic knowledge when making source attributions (Bayen, Nakamura, Dupuis, \& Yang, 2000; Spaniol \& Bayen, 2002). While reliance on such metacognitive knowledge can, in some cases, aid our source attributions when we are unsure of the correct response (i.e., when making source guesses; for a review, see Kuhlmann \& Bayen, 2016), it can also lead to errors of misattribution when the correct source is inconsistent with our goals or prior knowledge.

In the context of directed forgetting, like JOLs, the number of cognitive operations can be used as a cue to infer the source of a TBR or TBF item. According to the directed forgetting theories previously reviewed, TBR items are selectively rehearsed while TBF items may be actively suppressed, which creates a distinction in the memorability of these items. During the recognition task, when participants encounter an item with a strong memory trace, they are likely to use their knowledge of cognitive operations to attribute the item to a TBR source. In contrast, when an item with a weak memory trace is encountered (e.g., an accidentally remembered TBF item), participants are likely to attribute the item to the source associated with operations that would lead to low item memorability (i.e., the TBF cue). If participants can use this metacognitive knowledge to inform their decisions, then source monitoring of TBR and TBF items should be relatively equal. Evidence from Thompson and colleagues (2011) has shown that this is indeed the case: Participants are generally good at identifying the source of both TBR and TBF items, despite showing poorer item memory for TBF items. But reliance on cognitive operations in the context of directed forgetting has also been shown to produce errors of misattribution. As illustrated by Thompson and colleagues (2011), when participants mistook 
new items as old, they were more likely to attribute the new items to a TBF than to a TBR source (71\% vs. $29 \%$ of the time, respectively) presumably because TBF sources are associated with low item memorability. Such evidence implies that participants may indeed rely on their knowledge of cognitive operations to inform their attribution of items to TBR and TBF sources. Subjective experience of remembering. A final form of metacognition to be considered in the context of directed forgetting is the subjective experience of remembering. The procedure most commonly used to examine this recollective experience is the remember/know paradigm, in which participants distinguish between two states of awareness experienced during memory retrieval, referred to as "remembering" and "knowing” (Gardiner, 1988; Migo, Mayes, \& Montaldi, 2012; Tulving, 1985). A 'remember' judgment indicates that memory retrieval is accompanied by a vivid, conscious recollection of the event, such that participants become aware of features or contextual details that occurred at the time the event initially occurred. In contrast, a 'know' judgment is made when memory is characterized by a feeling of familiarity that lacks a conscious recollection of details or features associated with the event's initial occurrence. For example, we may encounter a familiar face and know that we have met that person, but cannot consciously recollect where we met them or who introduced us to them. According to Metcalfe and Dunlosky (2008), remember/know judgments represent a form of metacognition as they involve making an attribution about our memories and the degree of conscious awareness that we experience upon retrieval.

In the context of directed forgetting, the remember/know procedure has been a useful means for determining how participants' subjective experience of remembering and their conscious recollection differs as a function of TBR and TBF cues. Prior research has shown that deep processing manipulations during encoding tend to increase the degree of remember 
responses but not know responses, suggesting that increased distinctive processing is required to enhance conscious recollection (Gardiner, 1988). In an item-directed forgetting task, given that participants engage in selective rehearsal of TBR items and suppress processing of TBF items, it logically follows that their ability to consciously recollect contextual features should be more impacted by TBR than TBF cues. Evidence has largely supported this hypothesis (e.g., Basden \& Basden, 1996; Collette, Grandjean, Lorant, \& Bastin, 2014; Gardiner et al., 1994; Rizio \& Dennis, 2013, 2014, 2016). For example, Basden and Basden (1996, Experiment 3) administered an item-directed forgetting task and asked participants to give 'Recollect' ${ }^{4}$ or 'Know' responses for words they had previously studied, regardless of the cue that they were initially associated with. Results indicated that a directed forgetting effect (i.e., TBR $>$ TBF) occurred for recollection-based judgments, but not for know judgments. This was argued to be a byproduct of the increased distinctive processing applied to TBR over TBF items that, in turn, increased the subjective experience of remembering these TBR items. Taken together, the research in this area implies that the conscious state of recollection, as inferred by elevated remember judgments, contributes more to the directed forgetting effect than do memories that are based on feelings of familiarity, in which retrieval lacks conscious awareness.

\section{Interim Summary}

Taken together, the literature thus far has operationally defined intentional forgetting as an active process by which we strategically control the encoding of relevant and irrelevant information. The directed forgetting procedure is most often used to study this process, and is thought to be driven by mechanisms that selectively rehearse TBR items and actively suppress TBF items (Basden \& Basden, 1996; for reviews, see Anderson \& Hanslmayr, 2014; MacLeod,

\footnotetext{
4 'Recollect' responses were used in place of 'remember' responses to prevent confusion between the TBR memory cue and the 'remember' judgment (Basden \& Basden, 1996).
} 
1998). As well, participants seem able to use metacognitive knowledge about the differences in cognitive operations or item memorability associated with TBR and TBF items to inform their prospective and retrospective monitoring of directed forgetting performance via JOLs, source monitoring, or remember/know judgments (Friedman \& Castel, 2011; Thompson et al., 2011). With this foundational literature reviewed, the following sections will narrow the focus by examining how directed forgetting is influenced by healthy aging and emotional content - the two factors of primary interest to the current dissertation.

\section{Aging and Directed Forgetting}

The field of cognitive aging originally took interest in directed forgetting to further investigate theoretical models of inhibitory deficits in aging (Hasher \& Zacks, 1988). Since Zacks and colleagues' (1996) initial findings of age-related declines in the ability to intentionally forget, several studies have emerged that support these findings (e.g., Andrés, Van der Linden, \& Parmentier, 2004; Collette, Germain, Hogge, \& Van der Linden, 2009; Gallant \& Yang, 2014; Hogge, Adam, \& Collette, 2008; Sego, Golding, \& Gottlob, 2006). To determine the reliability of these age effects in the literature, Titz and Verhaeghen (2010) later meta-analyzed data from 24 independent samples that were derived from 10 directed forgetting experiments. Their results fell nicely in line with Zacks and colleagues' (1996) original findings: Directed forgetting effects in the literature were smaller in older (Cohen's $d=0.81$ ) relative to young adults $(d=1.17)$ and this age effect was larger for the item method of directed forgetting as compared to the list method of directed forgetting.

While such findings are often attributed to age-related declines in inhibition, evidence for age equivalence in intentional forgetting (e.g., Gamboz \& Russo, 2002) has challenged this hypothesis and suggests that changes in memory-related processes may also play a role. Gamboz 
and Russo (2002) directly tested this hypothesis by manipulating level of processing for each item during an item-directed forgetting task. In their study, young and older adults completed the item-directed forgetting task under one of three encoding conditions: "deep" processing (a pleasantness rating), "shallow" processing (letter counting), or a control group with no specific instructions. When items were encoded shallowly, young adults exhibited larger directed forgetting effects than older adults; however, when allowed to engage in deep processing, the age difference in directed forgetting was eliminated. The authors concluded that older adults' reduced directed forgetting likely reflected age-related differences in the ability to effectively encode TBR information into memory rather than a deficit in the suppression of TBF items. However, it is important to note that the deep encoding manipulation in this study primarily reduced young adults' intentional forgetting relative to that of the shallow encoding group. In fact, older adults' directed forgetting did not change between the shallow and deep encoding conditions. This suggests that the age equivalence in directed forgetting in the deep encoding group could not be solely attributed to improved intentional forgetting in older adults, but rather involved decreases in the forgetting of young adults.

A later study by Collette and colleagues (2014) further examined the role of memoryrelated processes in age-related directed forgetting by equating the quality of memory traces between young and older adults. When compared to young adults who completed a standard version of the task, older adults who were provided with encoding strategies (e.g., rote repetition, sentence generation, and mental imagery) as well as increased encoding time produced a similar magnitude of directed forgetting. When probed about they subjective recollection of their memory via remember/know responses, both age groups also showed a similar contribution of recollection-based processes to their directed forgetting but only if the memory trace quality was 
equal. When memory trace quality was not equated between age groups, older adults showed reduced directed forgetting and less conscious recollection of contextual detail for TBR items. Such results suggest that age-related declines in directed forgetting may be attributable not just to deficits in suppression (i.e., increased TBF item processing) but also to deficits in episodic memory processes such as the initiation of encoding strategies.

While evidence from neuroimaging could help to elucidate the underlying mechanism of age-related differences in directed forgetting performance, the literature on this topic is quite slim. To date, only one study has examined the effects of aging on the neural correlates of itemdirected forgetting (Rizio \& Dennis, 2014). Using fMRI, this study found that the brain mechanisms underlying intentional forgetting are quite different in young and older adults. Following a remember cue, older adults showed greater activity in the PFC (left middle frontal gyrus, left superior frontal gyrus) and parietal cortex (inferior parietal lobe); in contrast, young adults showed greater activity in posterior regions, such as the occipital cortex. Older adults' increased PFC activity following TBR cues was also associated with increased recognition of those items. The authors thus argued that older adults may recruit higher-order cognitive processes associated with the PFC to compensate for declines in encoding-related functions (e.g., self-initiated encoding strategies). This is consistent with literature on the cognitive neuroscience of aging, which has often indicated a marked anterior shift in brain activity during aging in both fMRI (Davis, Dennis, Daselaar, Fleck, \& Cabeza, 2008; Grady et al., 1994) and ERP studies (Friedman, 2003). This shift in neural recruitment is typically argued to reflect activity that compensates for impaired functioning in other regions of the brain and serves to aid performance (Grady, 2012; this topic is further detailed in Chapter 7). 
Age differences also emerged when Rizio and Dennis (2014) examined brain activity associated with suppressing TBF items. When compared to young adults, the older sample's activity suggested difficulty recruiting processes in the PFC that are typically engaged during intentional forgetting (Anderson \& Hanslmayr, 2014). Relative to young adults, older adults showed decreased activity in the right superior PFC whereas activity was intact for inhibitory related regions in the inferior parietal lobe. According to functional connectivity analyses, older adults' parietal activity following TBF cues was negatively correlated with activity in the MTL, which is important for encoding-related processes. This contrasts with the negative association between the DLPFC and MTL that is typically observed in young adults during intentional forgetting (as described on page 12; Anderson \& Hanslmayr, 2014; Rizio \& Dennis, 2013). The authors interpreted these divergent associations as an age-related shift in the 'hub' of activity that suppresses encoding of TBF items (i.e., PFC in young adults, parietal cortex in older adults). Altogether, Rizzio and Dennis’ (2014) study provides preliminary evidence for age-related divergence in brain networks supporting intentional forgetting.

Together, the reviewed literature on aging and directed forgetting provides insights into how the ability to strategically control the encoding and suppression of TBR and TBF items, respectively, can change with age. These studies also elucidate the circumstances under which these age effects are most likely to be observed or mitigated (e.g., Collette et al., 2014; Gamboz \& Russo, 2002). The neuroimaging literature - albeit scant - has also provided some information regarding age differences in the mechanisms that support intentional forgetting (Rizio \& Dennis, 2013). However, most of these studies have ignored the influence of emotion on directed forgetting during aging. This is surprising considering the large body of research outlined in Chapter 1 that suggests an age-related positivity effect in older adults' attention and memory 
(Mather \& Carstensen, 2005; Reed \& Carstensen, 2012; Reed et al., 2014). In contrast, as detailed in the following section, there are several studies examining the influence of emotion on young adults' directed forgetting.

\section{Emotion and Directed Forgetting}

Within the last decade, there has been an increased interest in understanding how emotion might modulate mechanisms of directed forgetting (e.g., Bailey \& Chapman, 2012; Brandt et al., 2013; Gallant \& Yang, 2014; Gallant \& Dyson, 2016; Hauswald et al., 2010; Kuehl et al., 2016; Liu, Chen, \& Cheng, 2017; Minnema \& Knowlton, 2008; Nowicka, Marchewka, Jednoróg, Yacikowski, \& Brechmann, 2011; Yang et al., 2012, 2013; Yang, Lei, \& Anderson, 2015; Zhang, Xie, Liu, \& Luo, 2016). This is not surprising given the influence that emotion has on information processing (for a review, see Pessoa, 2009). When emotional and neutral representations enter the visual field, they compete for selective attention. Which information comes out on top can depend on its stimulus-driven salience (bottom-up factors) or on the individual's goals and motivations (top-down factors; Beck \& Kastner, 2009). There is much research to suggest that, when pitted against neutral information, emotion captures attention, causing it to receive privileged processing (Pessoa, 2009). The degree to which emotion guides attention can be determined both by its degree of arousal (how calming or exciting) or its valence (how pleasant or unpleasant; Russell, 1980). When high in arousal (e.g., threatening stimuli), the stimulus-driven salience of emotional information promotes priority processing (Pessoa, 2009); however, when low in arousal but high in valence, top-down factors may come into play that selectively process emotional stimuli over others (Kensinger \& Corkin, 2004). Because of this priority processing, emotional events or stimuli are often more strongly embedded in memory than are stimuli lacking emotion. 
Based on the above logic, one might assume that emotional memories should be more difficult to forget. A review of the literature, however, shows that the influence of emotion on directed forgetting seems to depend on where the emotional stimuli fall on each of the valence and arousal continuums. For instance, Hauswald and colleagues (2010) tested the hypothesis that highly arousing negative memories should be more difficult to intentionally forget than neutral memories. Participants in this study completed an item-directed forgetting task for intermixed highly arousing negative images (e.g., a violent car crash) and non-arousing neutral images (e.g., a man standing in the snow) that were each followed by a TBR or TBF cue. Whereas intentional forgetting was successful for the neutral images, the directed forgetting effect was not observed for the highly arousing negative images. The authors concluded that highly arousing negative memories may be exempt from directed forgetting due to the privileged encoding they received over non-arousing memories. Other studies have shown that directed forgetting can be achieved for arousing negative images, but the capacity to do so is significantly reduced when compared to neutral baseline images (Nowicka et al., 2011; Otani et al., 2011). In contrast to these studies, however, Yang and colleagues (2012) examined the degree to which participants could intentionally forget negative and neutral images that were matched on mid-range levels of arousal to isolate the effects of stimulus valence. Interestingly, their results indicated that participants were just as able to intentionally forget emotional images as they were neutral.

Whereas the above studies focus on picture or image stimuli, similar effects of emotion on directed forgetting have been observed with verbal stimuli that vary in valence and arousal. Like the studies described above, when negative words are higher in arousal than the neutral baseline, they tend to reduce the magnitude of directed forgetting (e.g., Yang et al., 2015; although see Brandt, Nielsen, Holmes, 2013, for evidence of facilitative effects of negative 
arousing words on directed forgetting). In contrast, when emotional and neutral words are matched on mid-range to low levels of arousal, the directed forgetting effect does not seem to vary as a function of emotion (Berger, Crossman, \& Brandt, 2016; Gallant \& Yang, 2014; Patrick, Kiang, \& Christensen, 2015). To elucidate the contribution of valence and arousal, Gallant and Dyson (2016) directly compared the effect of negative, positive, and neutral words that were either high or low in arousal within the same item-directed forgetting task. Findings suggested that the directed forgetting effect was washed out under high arousing negative conditions due to reduced suppression of TBF words in this category, but the directed forgetting effect was intact for the other conditions.

Together, the literature on emotion and directed forgetting implies that high arousal stimuli (particularly of negative valence) may be the least likely to produce the directed forgetting effect. These results are what would be expected by the dual competition model, which provides a framework for understanding how emotion and cognition interact (Pessoa, 2009). According to this model, emotional content high in intensity (e.g., threatening stimuli) elicits an 'extreme mobilization' of resources toward the emotional item, resulting in privileged processing relative to non-emotional items. These resources, however, are shared with those needed for effective cognitive control. As a result, few resources may be left to engage the topdown processes required to implement the memory cue (i.e., TBR or TBF). In reconciling this conflict, the ability to effectively suppress TBF items is reduced. In contrast, stimuli low in arousal tend to elicit a 'soft' prioritization, which has a relatively weak influence on cognitive control processes, and therefore the emotional effect on directed forgetting may be attenuated or non-apparent (Pessoa, 2009). 
Whether these arousing stimuli are negative in valence also seems to play a role in reducing directed forgetting. This literature, however, primarily concerns young adults, who sometimes show a negativity bias in their emotional processing (Baumeister, Bratslavsky, Finkenauer, \& Vohs, 2001; Kensinger, 2008), which may play a role in why negative emotion had such a great influence in these studies. In contrast, stimuli high in valence but low in arousal do not seem to disrupt young adults' directed forgetting, suggesting that the top-down goals of the directed forgetting task may override the influence of emotional valence. Given older adults' motivational orientation toward positive stimuli, an interesting question concerns how directed forgetting of emotion would differ across age groups. Only just recently, however, did research begin to examine the influence of emotion on older adults' directed forgetting (e.g., Berger et al., 2016; Gallant \& Yang, 2014).

\section{Aging-Emotion Interactions on Directed Forgetting}

For my Master's thesis, I was motivated by the socioemotional selectivity theory (Carstensen, 1995) and evidence for emotion-cognition interactions in aging (Mather \& Carstensen, 2005) to examine how these factors would impact directed forgetting in young and older adults. Given older adults' positivity bias (Reed \& Carstensen, 2012), I was interested in determining whether positive emotion would facilitate encoding of TBR items, but hinder forgetting of TBF items for older relative to young adults.

A second goal of my thesis was to examine the metacognitive aspects of directed forgetting, particularly the ability to monitor the source of TBR and TBF items as well as how these processes would interact with aging and emotion. Source memory and monitoring is typically impaired in later life (Old \& Naveh-Benjamin, 2008); however, there is evidence that this may not be a general age-related deficit. For instance, when discriminating between two 
distinct sources (e.g., what they said vs. what another person said) older adults may be able to rely on metacognitive knowledge of cognitive operations (e.g., Hashtroudi, Johnson, \& Chrosniak, 1989; Kuhlmann \& Touron, 2011) or general knowledge (e.g., Mather et al., 1999) to inform source monitoring. Additionally, older adults have shown a better ability to remember the emotional as opposed to perceptual features of information (Hashtroudi, Johnson, \& Chrosniak, 1990) and show similar source memory to that of young adults when sources are emotional in tone (May, Rahhal, Berry, \& Leighton, 2005; Rahhal, May, \& Hasher, 2002). As such, consistent with Thompson and colleagues (2011), we predicted that young and older adults would show similar discrimination of TBR and TBF sources, which are each associated with distinct cognitive operations, and that there would be an additive benefit for older adults' source memory for positive items. When making source guesses (i.e., attributing new items to sources), we expected participants to show a bias to misattribute new items to TBF rather than TBR cues, given that TBF cues are associated with reduced item memorability. Such a pattern of findings would imply reliance on metacognitive knowledge of cognitive operations about which source ought to be assigned in the absence of source memory (Kuhlmann \& Bayen, 2016).

To address these goals, young and older adults were compared on a standard itemdirected forgetting task for intermixed positive, negative, and neutral words. We used emotional words that were relatively low in arousal, given evidence that older adults' positivity bias may be specific to nonarousing stimuli (Kensinger, 2008). To index source memory, a standard source monitoring task was used in which participants assigned items to TBR, TBF, or new sources during recognition, as in the procedure outlined by Thompson et al. (2011; but also see Goernert et al., 2011; MacLeod, 1999; Otani et al., 2011, for similar approaches). When adopting this methodology in our study (Gallant \& Yang, 2014), a general age-related reduction in item- 
directed forgetting emerged, supporting prior findings (Titz \& Verhaeghen, 2011). Contrary to expectations, however, age and emotion did not differentially influence directed forgetting. Rather, both groups remembered and intentionally forgot positive and negative words just as well as neutral words (Gallant \& Yang, 2014).

Although the null effect of emotion on older adults' directed forgetting is seemingly at odds with the socioemotional selectivity theory (Carstensen, 1995), the results may be reconcilable within a cognitive control account of the age-related positivity effect (Reed \& Carstensen, 2012). As described earlier, this account suggests that the age-related positivity effect is motivational in nature and driven by top-down goals. When cognitive control is low or other top-down demands override older adults' emotional goals, the positivity effect is therefore reduced (e.g., Knight et al., 2007; Mather \& Knight, 2005). This is supported by a recent metaanalysis of 100 empirical studies from the literature ( $N=7,129$ samples) showing that the positivity effect is larger in magnitude when cognition is unconstrained by experimental tasks (Reed et al., 2014). Thus, in Gallant and Yang (2014), the top-down processes required to activate TBR and TBF processes likely conflicted with older adults' chronically activated emotional goals. Regardless of the mechanism, the results support the idea that both young and older adults can strategically control encoding of TBR and TBF items even in the face of emotional stimuli. Importantly, this effect that has recently been replicated (Berger et al., 2016).

In contrast to the directed forgetting results, emotion differentially influenced source monitoring across age groups. Relative to young adults who showed similar source memory for both TBR and TBF items across emotion conditions, older adults' performance was differentially influenced by emotion. Whereas older adults' source memory of TBR and TBF items was similar for neutral items, they showed enhanced source memory for positive TBR items and 
impaired source memory for positive TBF items. The opposite pattern was observed for negative items. We explained these results within the framework of socioemotional selectivity suggesting that older adults' preference to remember positive information may have enhanced their ability to bind TBR cues with positive words, thus enhancing their source memory for these items. In contrast, cues to forget or suppress positive information would be inconsistent with their emotion focused goals, reducing the likelihood of binding positive items with TBF cues. Instead, older adults showed a tendency to misattribute positive TBF words to TBR sources.

When making source guesses (i.e., attributing new items to TBR and TBF sources), young adults showed the expected tendency to attribute a higher proportion of new words to TBF rather than TBR sources, regardless of emotion. This implies that young adults were indeed relying on their metacognitive knowledge of cognitive operations during source monitoring. In contrast, whereas older adults showed a tendency to over-attribute neutral and negative new words to TBF sources, they showed no such differentiation in their misattributions to positive new words. Rather, older adults were more likely to misattribute positive new words to TBR sources. This pattern of results suggests that positive information may have interfered with older adults' ability to use metacognitive knowledge of cognitive operations during source guessing. One possible explanation for this misattribution bias is that older adults may perceive TBR sources as higher in value than TBF sources and - consistent with their positivity bias - adopt a theory that positive information must belong to the source with higher value or relevance for memory. This, in turn, may make it more difficult for older adults to attribute a positive item to a source that is inconsistent with their emotion-focused goals.

In sum, the results of this thesis contributed novel findings to the literature. First, despite an age-related preference for positive information in attention and memory (Reed \& Carstensen, 
2012), the ability to strategically control emotional TBR and TBF memories is intact in later life. In contrast, aging and emotion differentially influenced source monitoring of TBR and TBF cues. While older adults showed enhanced source memory for positive TBR words in line with the age-related positivity effect (Reed \& Carstensen, 2012), their source memory for positive TBF words was impaired. This may imply that older adults had difficulty making correct source attributions for information that is inconsistent with their emotional goals. Moreover, their reliance on metacognitive knowledge of cognitive operations when making source guesses was disrupted for positive words. Although interesting, there are many questions that remain open with regard to young and older adults' use of metacognitive knowledge during directed forgetting of emotion, as well as with regard to the neural correlates associated with these processes. The goal of the current dissertation is thus to extend the findings of my M.A. thesis by addressing these questions, which are further detailed in the next chapter. 


\section{Chapter 3: Summary of Dissertation Aims}

\section{Objectives}

The overarching goal of this dissertation is to extend the findings of my M.A. thesis (Gallant \& Yang, 2014) by further examining the metacognitive aspects and neural correlates that underlie young and older adults' intentional forgetting of emotional information. To reiterate, my M.A. thesis demonstrated that (1) young adults were generally more efficient at directed forgetting than older adults; (2) despite this age difference, both age groups demonstrated directed forgetting for emotional words, implying intact control over emotional TBR and TBF words in later life; (3) whereas young adults' source attributions did not vary by

emotion, older adults had better source attributions for positive TBR items than for positive TBF items; and (4) older adults' use of metacognitive knowledge when making retrospective source guesses seemed to be disrupted by positive information.

Several research questions follow from these findings. While older adults' retrospective use of metacognitive knowledge during source guessing was seemingly impaired by positive information, it remains unclear whether their prospective metacognitive judgments would be similarly affected by emotion in the context of a directed forgetting task. This is an important question to address considering the importance of metacognitive monitoring for controlling cognitive processes (Hertzog \& Dunlosky, 2011). A second question is whether older adults' bias to misattribute positive items to TBR sources is the result of a bias to assign positive items to sources that are perceived as higher in value or relevance. A final question concerns age differences in the neural correlates of directed forgetting performance. Although Rizio and Dennis' (2014) findings shed light on the spatial aspects of young and older adults' directed 
forgetting in the brain, their results cannot speak to the temporal dynamics of this effect or how these correlates would vary with emotion.

\section{Experiments}

To address these questions, three experiments were conducted in which young and older adults completed variations of an item-directed forgetting task for positive, negative, and neutral words. In Experiment 1, I examined age differences in prospective metacognitive monitoring of emotional directed forgetting using a JOL procedure embedded within the encoding phase of the item-directed forgetting task (similar to Friedman \& Castel, 2011). During recognition, young and older adults again attributed items to TBR, TBF, or new sources to determine the replicability of the age and emotion interaction on source monitoring (Gallant \& Yang, 2014). In Experiment 2, I examined the hypothesis that older adults' source misattribution bias may be driven by a tendency to misattribute positive items to sources that are perceived as higher in value. In this experiment, young and older adults completed a value-based version of the itemdirected forgetting task in which conceptual cues to 'remember' or 'forget' were replaced with positive or negative values (+10 vs. -10$)$, respectively. This also allowed for examination of whether intentional forgetting of emotional words could extend beyond a directed forgetting paradigm to instances where cues signal a gain or loss of value. Finally, in Experiment 3, I examined brain activity underlying young and older adults' attempts to remember TBR items and suppress TBF items, using the ERP technique. Additionally, this experiment further examined the metacognition of emotional directed forgetting and source monitoring by probing participants' subjective experience of remembering using an adapted version of the remember/know procedure (Tulving, 1985). 
The dissertation is organized as follows. The ensuing chapters begin by outlining the General Method common to each experiment, followed by a detailed description of the three experiments described above. Each experiment chapter will provide an overview of the literature specific to that experiment, further highlighting the rationale and to-be-tested hypotheses. After detailing Experiments 1 to 3, the General Discussion will tie the results together and discuss the broader theoretical and empirical implications of this work. Limitations of the current research and avenues for future research will be provided. 


\section{Chapter 4: General Method}

For simplicity, this chapter details the methods and procedures common to the three experiments in this dissertation. Methods that are experiment-specific will be further described in each experiment's respective chapter. All procedures adhered to ethical standards in conducting human psychological research and were approved by the Ryerson University Research Ethics Board (see Appendices I-XI for ethics related materials).

\section{Participants}

Sample size estimates. A-priori sample size estimates were calculated using $\mathrm{G}^{*}$ Power 3.1.9.2 (Buchner, Erdfelder, Faul, \& Lang, 2014). Using a repeated measures design with $\alpha$ set at .05 , the $\mathrm{G}^{*}$ Power analysis indicated that a sample of $n=24$ per group would provide $90 \%$ power to detect a small-medium within-between interaction $\operatorname{effect}(f=.25)$ with a 2 (age: young, old) $\times$ 3 (valence: negative, positive, neutral) $\times 2$ (cue: TBR, TBF or $+10,-10)$ mixed design. To further enhance power, samples slightly above this estimate were acquired in all experiments.

Participant recruitment. Healthy young (age range 17-29) and older adults (ages 65 and above) participated in Experiments 1 to 3. Young participants were recruited from the undergraduate participant pool through the Introduction to Psychology courses at Ryerson University whereas older adults were recruited from the Ryerson Senior Participant Pool. Participants were compensated for their participation based on the length of the experiment. For a 1-hour session (Experiments 1-2), young adults received one credit toward their final grade of the Introduction to Psychology course and older adults received \$12 CAD. For a 2-hour session (Experiment 3), young adults received two credits and older adults received \$20 CAD. All participants provided informed consent prior to commencing their participation in the experiments. Participants in Experiments 1 and 2 were tested in the Psychology Research and 
Training Centre at Ryerson University. Those in Experiment 3 were tested in the EEG Lab of the Institute for Stress and Wellbeing Research at Ryerson University.

Inclusion/exclusion criteria. Across experiments, participants were required to meet eligibility criteria for their data to be included in analyses. Participants' data were excluded and replaced based on the following health-related criteria: (1) history of neurological abnormalities or disorders (e.g., traumatic brain injury, stroke, dementia, prolonged periods of unconsciousness); (2) a current diagnosis of uncontrolled medical conditions that might affect cognitive performance (e.g., diabetes, cardiovascular diseases); (3) a current diagnosis of a mood disorder (e.g., depression or anxiety); (4) current prescription of medications that impact mental functioning; and (5) visual impairments.

Participants were also excluded based on their performance on a battery of cognitive and emotional assessments including: (1) a score over six on the Short Blessed Test (SBT; Katzman et al., 1983) suggesting presence of cognitive impairment (older adults only); (2) a score of less than 20 on the Shipley Institute of Living vocabulary test (Shipley, 1940), suggesting poor proficiency with the English language; (3) a score of 28 or over on the depression sub-scale of the 21-item Depression Anxiety Stress Scale (DASS-21; Lovibond \& Lovibond, 1995) suggesting extremely severe depressive symptoms within the past week; and/or (4) a score of 20 or over on the anxiety sub-scale of the DASS, suggesting extremely severe anxiety symptoms within the past week. Participants were excluded based on these latter two criteria due to findings that depression and anxiety may elicit different emotional biases in attention and memory (e.g., Dalgleish et al., 2003; MacLeod \& McLaughlin, 1995). 


\section{Materials}

Stimuli. The same list of 120 words was used in Experiments 1-3. These words were selected from the Affective Norms for English Words (ANEW) database (Bradley \& Lang, 1999) based on valence and arousal norms. Ratings for the valence and arousal dimensions ranged from 1 to 9 , with 1 representing words high in negative valence and low arousal and 9 representing words high in positive valence and high arousal, respectively. Importantly, mean valence for each condition significantly differed from the other two conditions (i.e., negative $<$ neutral $<$ positive) and the range did not overlap across conditions. To isolate the effects of stimulus valence, all three conditions were matched on arousal in addition to word length and word frequency (see Table 1 for stimulus characteristics, and Appendix X for the list of stimuli). Table 1

Characteristics of the Stimuli used in Experiments 1-3

\begin{tabular}{lcccccc}
\hline & \multicolumn{2}{c}{ Positive $(n=40)$} & \multicolumn{2}{c}{ Negative $(n=40)$} & \multicolumn{2}{c}{ Neutral $(n=40)$} \\
\cline { 2 - 7 } Characteristic & $M(S D)$ & Range & $M(S D)$ & Range & $M(S D)$ & Range \\
\hline Valence $^{\mathrm{a}}$ & $7.39(0.40)$ & $6.7-8.1$ & $2.64(0.61)$ & $1.6-3.6$ & $5.01(0.55)$ & $4.0-6.0$ \\
Arousal $^{\mathrm{b}}$ & $4.38(0.63)$ & $3.0-5.4$ & $4.45(0.51)$ & $3.3-5.8$ & $4.20(0.40)$ & $3.4-5.0$ \\
Word length $^{\mathrm{c}}$ & $5.97(0.26)$ & $3.0-9.0$ & $5.70(0.25)$ & $3.0-10.0$ & $6.02(0.26)$ & $3.0-9.0$ \\
Frequency $^{\mathrm{d}}$ & $49.10(47.02)$ & $1.0-216.0$ & $49.15(64.80)$ & $3.0-277.0$ & $51.10(69.30)$ & $1.0-244.0$ \\
\hline
\end{tabular}

Note: Ratings based on norms from the ANEW database (Bradley \& Lang, 1999). Valence categories ${ }^{\mathrm{a}}$ differed on mean valence $(p s<.001)$, ${ }^{\mathrm{b}}$ matched on mean arousal $(p s>.07)$, ${ }^{\mathrm{c}}$ matched on mean number of letters in each word ( $p s>.30)$, and ${ }^{d}$ matched on mean word frequency $(p s>.88)$.

Words were divided into two lists of 60, which were counterbalanced as 'old' and 'new' lists across participants. Each list was further divided into two sub-lists of 30 words, each counterbalanced as TBR- or TBF-cued words (or +10 and -10 cued words), counterbalanced across participants. All list divisions were matched on valence, arousal, word length and word frequency. In addition to the experimental stimuli, an additional nine neutral words were 
selected, three to be used during practice trials and six to be used as buffers evenly placed at the beginning and end of the encoding list. These words were not included in the recognition list.

Stimulus delivery software. The experimental task was programmed and delivered using E-Prime 2.0 software (Psychology Software Tools, Pittsburgh, PA) in Experiments 1 and 2. The directed forgetting task in Experiment 3 was programmed and delivered using Presentation software (Neurobehavioral Systems, Inc., Berkeley, CA). Stimuli were presented in black size-16 Arial font against a white background on a 17" PC laptop computer in Experiments 1-2 and a 24" PC desktop computer in Experiment 3.

Item-directed forgetting task. Across experiments, an item-directed forgetting task was used to examine the influence of aging and valence on cognitive control and its metacognitive aspects. As described in Chapter 2, this task required participants to study a series of individually presented words, each followed by a TBR or a TBF cue. In all experiments, a source monitoring procedure was used during the recognition phase in which participants indicated whether an item was one they were supposed to remember, was one they were supposed to forget, or was new (for similar methods see Gallant \& Yang, 2014; MacLeod, 1999; Otani et al., 2011; Thompson et al., 2011). This is different from traditional directed forgetting paradigms that use a recall or recognition task in which participants are asked to distinguish old items from new items, regardless of the cue they were originally paired with. Importantly, this source monitoring procedure has been shown to produce comparable directed forgetting effects to those in the standard old/new recognition task (Gallant \& Yang, 2014; Thompson et al., 2011). A variation of this task was used across experiments to address the specific research questions of each study, with study-specific procedural details provided in the later chapters. 
Cognitive, emotional, and demographic assessments. A battery of paper-pencil questionnaires was administered following the recognition phase of all experiments to collect information on individual differences with respect to cognitive, emotional, and demographic factors. For factors that showed significant group differences, further analyses were conducted to determine whether individual differences in these variables (e.g., processing speed, mood state, vocabulary) may be associated with the dependent variables that also showed age differences.

\section{Cognitive measures.}

Digit Symbol Substitution Test (DSST). The DSST is a neuropsychological test from the revised version of the Wechsler Adult Intelligence Scale (WAIS-R) and measures processing speed (Wechsler, 1981). The task consists of nine digits, each paired with a specific symbol. During the task, participants matched as many of the symbols with their corresponding digits as possible. The total number of correct solutions provided an index of processing speed. In all experiments, the DSST was used during the filler period between the encoding and recognition phases of the directed forgetting task to wipe working memory and prevent rehearsal.

Shipley Institute of Living Vocabulary Test. This 40-item vocabulary test was adopted from the Shipley Institute of Living Scale (Shipley, 1940) to assess proficiency with the English language. As verbal stimuli were used in these experiments, it was important to ensure that low memory scores were not driven by poor knowledge of English vocabulary. During the test, participants chose one of four words that had the same meaning as a prompt word.

Short Blessed Test (SBT). The SBT is a weighted six item scale designed to measure dementia-related impairment in an aging population. It has been shown to reliably discriminate among mild, moderate, and severe cognitive dysfunction (Katzman et al., 1983). The items on the scale evaluate orientation to time, memory, and concentration. Each question on the scale is 
orally administered and participant responses are recorded and scored. Scores can range from 0 to 28 , with those in the 0 to 6 range being considered "normal".

\section{Emotion assessments.}

Depression Anxiety Stress Scales (DASS-21). The DASS-21 scale was designed to measure depression, anxiety, and stress over the past seven days (Lovibond \& Lovibond, 1995). The depression sub-scale includes seven items that measure dysphoria, sense of hopelessness, self-deprecation, devaluation of life, lack of interest/motivation, and anhedonia. The anxiety subscale includes items that measure autonomic arousal, skeletal/muscle effects, and subjective experience of anxious affect. The stress sub-scale includes items that target difficulty with relaxing, the tendency to be irritable/over-react, and nervous arousal. Participants were asked to indicate the degree to which each statement relates to them over the last seven days using a 4point Likert scale ranging from 0 ("did not apply to me at all") to 3 ("applied to me very much or most of the time"). Scores for each scale were summed and multiplied by two with a possible range of 0 to 42 for each sub-scale. These scores were then compared against severity ratings for each dimension to determine symptom severity as normal, mild, severe, or extreme. In the current experiments, only estimates of depression and anxiety were analyzed, given prior research suggesting that such symptoms can elicit different emotional biases during cognitive processing (e.g., Dalgleish, 2003; MacLeod \& McLaughlan, 1995).

Positive and Negative Affect Schedule (PANAS). The 20-item PANAS quantifies experience with two primary dimensions of mood—positive and negative affect (Watson, Clark, \& Tellegan, 1988). The scale consists of 10 positive emotions and 10 negative emotions. During the task, participants were asked to rate the extent to which they were experiencing each emotion on a 5-point Likert scale ranging from 1 "very slightly or not at all" to 5 "extremely". The sum of 
ratings for positive items was used to index positive affect whereas the sum of ratings for negative emotion items provides an index of negative affect. Possible scores range from 10 to 50 for each dimension with larger scores indicating higher levels of positive or negative affect.

Demographic and health questionnaire. All participants completed a background questionnaire including queries related to age, gender, and years of formal education (not including continuing education). Health questions inquired about prior and/or current diagnoses of medical conditions (e.g., heart conditions, neurological disorders, and/or psychiatric disorders) and current medication use.

\section{Experimental Design}

All studies adopted a $3 \times 2 \times 2$ mixed factorial design that included valence (negative, positive, neutral) and cue (TBR, TBF or $+10,-10)$ as the within-subject variables and age group (young, older) as the between-subjects variable. In all experiments, dependent variables in the analysis of item recognition performance included hits to old items, false alarms to new items, discrimination sensitivity $\left(A^{\prime}\right)$ and response bias $\left(B^{\prime}{ }_{D}\right)$. In analyzing source monitoring, dependent variables included conditional source identification scores and misattributions of new items to sources. Deviations from this design are detailed within specific experiment chapters.

\section{Data Analysis Approach}

All statistical analyses were conducted using IBM SPSS version 24 (IBM Corp., 2014). Across experiments, results were interpreted in terms of significance $(\alpha=.05)$ and effect size. For ANOVAs, effect size was inferred using partial eta squared $\left(\eta_{p}^{2}\right)$, which indicates the proportion of variance explained by a variable that is not explained by other variables in the model. For independent and dependent $t$-tests between two means, Cohen's $d$ was used as the effect size estimate, with $.20, .50$, and .80 corresponding to small, medium, and large effects, 
respectively. In instances where the assumption of sphericity was violated, the GreenhouseGeisser procedure was used to correct the degrees of freedom of the F-distribution. To unpack significant interactions, planned comparisons were made that were derived from our prior research (Gallant \& Yang, 2014) as well as a-priori hypotheses. When making post-hoc comparisons, Type I errors were controlled by adjusting alpha-levels using Bonferroni corrections. Analyses varied across experiments mainly in terms of the within- and betweensubjects factors included in the models and thus are further detailed within each chapter. The dependent variables listed below were commonly analyzed across experiments.

Item recognition performance. Analysis of recognition for all TBR and TBF items (or +10 and -10 items in Experiment 2), regardless of the source attributed to the item (i.e., the memory cue), provided a measure of overall item recognition performance. This aggregate measure of item-based hits was generated by combining responses made to each source or cue type across each valence and cue condition (for a similar approach see Gallant \& Yang, 2014; Thompson et al., 2011). Similarly, false alarm rates were defined as the proportion of new items mistakenly recognized as 'old', regardless of source. In this analysis, it was not possible to disentangle false alarms according to cue because new items do not have such a designation. As such, false alarms were calculated by combining $\mathrm{R}$ and $\mathrm{F}$ responses to new items across valence conditions to create an aggregate index of 'old' responses to new items.

Using hit and false alarm rates, discrimination sensitivity and response bias were calculated to gauge participants' ability to distinguish signal (i.e., previously studied items) from noise (new items) during the recognition task. According to signal detection theory, during a recognition task, participants base their memory judgment for each item (i.e., old vs. new) on a scale of strength, which they use to adopt a decision criterion or threshold for accepting or 
rejecting an item as 'old' (Macmillan \& Creelman, 2005). If the strength of the item is perceived as high, the item will be accepted as 'old', indicating a signal was present. If the strength of the item is perceived as low, the participant will reject the item by responding 'new' to indicate that a signal was absent. These decisions, however, can also be subject to bias in which a participant displays a general proclivity to respond 'old' or 'new' that may be independent of memory. To address issues of item recognition in the current experiments, discrimination sensitivity $\left(A^{\prime}\right)$ and response bias $\left(B{ }_{D}\right)$ were calculated according to the formulas outlined by Donaldson (1992). These non-parametric measures were used in place of parametric indices (e.g., $d$ ' and $c$ ) as they can be calculated with hit or false alarm rates of 1 or 0 . Discrimination sensitivity values range from chance performance $\left(A^{\prime}=0.5\right)$ to perfect performance $\left(A^{\prime}=1.0\right)$. Response bias or $B^{\prime \prime}{ }_{D}$ values range from a liberal bias (negative values) where less signal is required to respond 'old' to a conservative bias (positive values) where more signal is required to respond 'old'.

In analyzing item recognition performance, hits were analyzed in a series of mixed-model ANOVAs as a function of the within-subject factors of cue (TBR vs. TBF or +10 vs. -10 ) and valence (negative, positive, neutral) as well as the between-subjects factor of age (young vs. older). Since false alarms cannot be distinguished on the basis of cue, this factor was removed from the analysis of false alarms, $A$, and $B{ }_{D}$ resulting in 3 (valence) $\times 2$ (condition) ANOVAs. If $B{ }_{D}$ scores fell above or below zero (i.e., neutral bias), one-sample $t$-tests were conducted to confirm whether $B{ }_{D}$ scores differed significantly from zero. If scores were significantly greater than zero, they were considered to reflect a conservative bias, whereas scores that fell significantly below zero were considered to reflect a liberal bias. If scores did not differ from zero, bias was considered neutral. 
Source monitoring performance. Source recognition was indexed using the singlesource conditional source identification measure (CSIM), which calculates the mean proportion of words attributed to the correct source divided by the total number of items that were correctly recognized as old (Bayen et al., 1996; Foley, Johnson, \& Raye, 1983). For example, source monitoring for TBR items would be calculated with the following formula: TBR items identified as TBR / (TBR items identified as TBR + TBR items identified as TBF). Because the CSIM is dependent on the proportion of items attributed to the alternative source, it provides information about the degree to which old items were misattributed to incorrect sources (i.e., the inverse of the CSIM). For example, if the above equation indicated that $77 \%$ of items were correctly attributed to a TBR source, then $23 \%$ of those items were misattributed to a TBF source.

In addition, misattributions of new items to sources or source false alarms (e.g., new items identified as TBR) were analyzed to provide an index of source guessing (consistent with prior source monitoring practice, e.g., Mather et al., 1999). The CSIM scores and source false alarms were separately submitted to a mixed-model ANOVA as a function of the within-subject factors of cue and valence as well as the between-subjects factor of group.

Analysis of individual differences. Independent-sample $t$-tests were used to test for between-group differences on the emotional and cognitive assessments. For variables that showed group differences, correlations were calculated within groups to determine whether performance on the cognitive or emotional measure corresponded with performance on the dependent variables. These analyses were performed only if age differences were also observed in the dependent variables (e.g., CSIMs). If the correlation was significant, mediation analyses were performed (Hayes, 2012), to determine whether these cognitive or emotional variables could be mediating the effect of age on the dependent variables. 


\section{Chapter 5: Age Differences in Metacognitive Predictions of Emotional Directed Forgetting Experiment 1}

Our previous study (Gallant \& Yang, 2014) suggests that, in the context of directed forgetting, older adults have greater source recognition for positive TBR items than for positive TBF items. When making source guesses, older adults also exhibit a tendency to misattribute positive TBF and new items to TBR sources when compared to young adults. These results thus suggest that older adults' ability to use their metacognitive knowledge of cognitive operations to inform their source monitoring of TBR and TBF items may be disrupted by the presence of positive information. As previously mentioned, one possible reason for this misattribution bias may be that older adults prefer to attribute positive items to TBR sources, as these cues may be perceived as higher in value or relevance for memory. Thus, given their positivity bias, it is in line with their goals to attribute such sources to positive items.

Regardless of the mechanism driving our previous results (Gallant \& Yang, 2014), the data only allow us to infer that positive information influences older adults' retrospective monitoring for sources that vary in item memorability. Thus, in Experiment 1, I examined whether emotion would similarly influence older adults' ability to make prospective judgments about their learning of TBR and TBF items using a JOL procedure (similar to Friedman \& Castel, 2011). This is an important question to address, considering that these two forms of monitoring share many underlying features. As outlined in Chapter 2, both processes are core features of metamemory or metacognition (Metcalfe \& Dunlosky, 2008). Although inferred at different time points, these judgments both require evaluation of the contextual features of information in memory (e.g., differences in item memorability or cognitive operations) to effectively monitor performance (Koriat, 1997; Kuhlmann \& Bayen, 2016). In addition, both 
forms of monitoring can be influenced by factors beyond the contents of memory. For instance, as described by Johnson et al. (1993), source attributions are inferred based on characteristics of memories in combination with judgments about where that information is most likely to have come from. As such, these attributions can be influenced by stereotypes (Mather et al., 1999), schemas (Bayen al., 2000; Spaniol \& Bayen, 2002), current goals, or beliefs (Johnson et al., 1993; Kuhlmann \& Bayen, 2016; Mitchell, 2016). In contrast, JOLs typically involve making a prospective judgment about how likely the learned information will later be remembered (Metcalfe \& Dunlosky, 2008). Like source monitoring, these judgments are based on features of the stimuli entering memory (e.g., extrinsic cues; Koriat, 1997) but can also be influenced by our goals and beliefs in our capacity to remember (Hertzog, Dixon, \& Hultsch, 1990).

In the context of aging, metacognitive monitoring of learning tends to be spared (for reviews, see Hertzog \& Dunlosky, 2011; Hertzog \& Hultsch, 2002). For example, Robinson, Hertzog, and Dunlosky (2006) examined young and older adults' ability to monitor learning of word pairs. During the experiment, participants studied a series of paired-associates and provided a JOL after each item. On a subsequent memory task, older adults showed poorer associative memory than young adults (Old \& Naveh-Benjamin, 2008); however, both young and older adults' JOLs were predictive of memory performance, implying spared metacognitive monitoring in the older group. Similar age invariance in JOLs and their relative accuracy has since been found for images (e.g., Haber, 2012), as well as emotional and non-emotional words (Tauber \& Dunlosky, 2012). Related to the current experiment, Kuhlmann and Touron (2011) have also shown that older adults are just as able as young adults to rely on extrinsic cues about item memorability to inform their JOLs. In their study, both age groups monitored information from two sources: information presented in bold or italic font. Differences in item memorability 
across sources were created by selectively repeating words from one source. Results indicated that both groups' JOLs were sensitive to differences in item memorability across sources, suggesting that use of extrinsic cues to guide JOLs is spared by aging.

Taken together, in contrast to the often-observed age deficits in source monitoring (Johnson et al., 1993), there seems to be age equivalence in the ability to monitor learning via JOLs (Hertzog \& Hultsch, 2002). Although young and older adults' JOLs are sensitive to extrinsic cues concerning item memorability or cognitive operations (Friedman \& Castel, 2011; Kuhlmann \& Touron, 2011), it is not known whether this sensitivity would be observed for emotional information of varying sources. This question is of interest to this dissertation, given our findings that positive valence disrupts older adults' ability to use knowledge of cognitive operations to guide retrospective monitoring of TBR and TBF sources (Gallant \& Yang, 2014). To address this question in the current experiment, young and older adults completed an itemdirected forgetting task for positive, negative, and neutral words. Modeled after Friedman and Castel (2011), participants provided JOLs after each memory cue, predicting the likelihood of remembering the item that they had just studied. During recognition, a source monitoring task was administered in which participants attributed items to a TBR, TBF, or new source.

\section{Hypotheses}

As described above, both young and older adults can use extrinsic cues to guide their metacognitive JOLs. As such, I expected that both age groups would show a similar ability to monitor learning of TBR and TBF information. This would be illustrated by higher JOLs assigned to TBR than to TBF items. Regarding the relative accuracy of these JOLs, I expected that both young and older adults' JOLs would be predictive of subsequent recognition (inferred via above chance gamma correlations), specifically for TBR items. However, given that 
Friedman and Castel (2011) did not find that JOLs for TBF items were predictive of subsequent recall, this hypothesis was left open.

In contrast, source monitoring performance was expected to replicate our previous results (Gallant \& Yang, 2014). Young adults were expected to show similar rates of source memory for TBR and TBF items regardless of valence. In terms of their source misattributions, a higher tendency to attribute new items to the source with low item memorability (i.e., a TBF source) was expected. This would imply reliance on metacognitive knowledge of cognitive operations during source monitoring. In contrast, I expected that valence would differentially influence the older group's ability to attribute items to TBR or TBF sources. Specifically, relative to the other valence and cue conditions, older adults were expected to show better source memory for positive TBR items than for positive TBF items. Their misattributions of new items to sources were also expected to vary with emotion in the form of a bias to attribute positive new items to TBR sources relative to the other conditions. This would replicate our (Gallant \& Yang, 2014) previous pattern of results and imply that positive emotion disrupted their ability to rely on metacognitive knowledge when making source guesses.

\section{Method}

\section{Participants}

The final sample included 36 young adults (ages 17-28) and 35 older adults ${ }^{5}$ (ages 6585). All participants completed the battery of assessments outlined in the General Method section (pages 40-42). These data showed that older adults were more educated, had higher positive affect, fewer symptoms of depression and anxiety, higher vocabulary, and slower processing speed. One older adult was excluded and replaced for having experienced a stroke in the last year

\footnotetext{
${ }^{5}$ The data of one older adult was excluded at the analysis stage as their E-Prime data file was found to be corrupted when merging the data.
} 
as well as 11 young adults for scoring too high on the anxiety and/or depression subscales of the DASS-21. The final sample characteristics are displayed in Table 2.

Table 2

Characteristics of the Final Sample in Experiment 1

\begin{tabular}{|c|c|c|c|c|}
\hline & $\begin{array}{l}\text { Young Adults } \\
\quad(n=36)\end{array}$ & $\begin{array}{l}\text { Older Adults } \\
\quad(n=35)\end{array}$ & & \\
\hline Characteristic & $M(S D)$ & $M(S D)$ & $p$-value & $d$ \\
\hline Age in Years & $19.25(2.16)$ & $71.66(6.00)$ & $<.001 * *$ & -11.68 \\
\hline Male / Female Ratio & $8 / 28$ & $4 / 31$ & .225 & - \\
\hline Years of Education & $13.40(1.52)$ & $15.50(3.25)$ & $.001 *$ & -0.83 \\
\hline Positive Affect ${ }^{a}$ & $26.02(7.84)$ & $36.06(6.87)$ & $<.001 * *$ & -1.36 \\
\hline Negative Affect ${ }^{a}$ & $13.31(3.39)$ & $11.89(3.07)$ & .069 & 0.44 \\
\hline Depression $^{b}$ & $8.17(5.02)$ & $4.91(4.32)$ & $.005^{*}$ & 0.69 \\
\hline Anxiety ${ }^{b}$ & $5.83(5.02)$ & $3.50(3.71)$ & $.029 *$ & 0.53 \\
\hline Shipley Vocabulary & $28.00(3.67)$ & $34.91(3.74)$ & $<.001 * *$ & -1.86 \\
\hline $\operatorname{DSST}^{\mathrm{c}}$ & $83.50(11.74)$ & $64.46(15.38)$ & $<.001 * *$ & 1.39 \\
\hline Short Blessed Test & - & $.91(1.483)$ & - & - \\
\hline
\end{tabular}

Note. Between-group comparisons were made using independent-sample $t$-tests apart from gender ratio, which was examined using Pearson's chi square. ${ }^{\text {a }}$ Measured with the Positive and Negative Affect Schedule; ${ }^{b}$ Measured with the DASS-21; ${ }^{c}$ Scores reflect number of correct solutions. DSST $=$ Digit Symbol Substitution Test. ${ }^{*} p<.05,{ }^{*} * p<.001 . d=$ Cohen's $d$ for standardized effect size estimates of the mean difference between groups.

\section{Experimental Design}

The design was a 3 (valence) $\times 2$ (cue) $\times 2$ (age group) mixed factorial. The dependent variables in this experiment diverged from those reported in the General Method. The encoding phase of the directed forgetting task was modified to include JOLs after each cue, which were made on a scale ranging from 1 (will forget) to 10 (will remember). As such, in addition to item recognition and source monitoring performance, dependent variables included metacognitive 
monitoring assessed by means of JOLs to each word type and the relative accuracy of these JOLs (i.e., gamma correlations) for predicting subsequent recognition performance (see footnote \#4 on page 15 for a detailed explanation of Gamma correlations for indexing JOL relative accuracy).

\section{Procedure}

Upon arrival, participants provided informed consent for their participation after which they were seated in front of a laptop computer and instructions were provided. They were asked to study each word and to remember words followed by a remember cue ("RRRR") and to forget words followed by a forget cue ("FFFF"). Participants were further told that after each cue they would need to provide a JOL by predicting the likelihood that they would be able to remember or forget the word that they had just studied on a scale of 1 (will forget) to 10 (will remember), with a JOL of 1 indicating $0 \%$ chance of memory and a JOL of 10 indicating $100 \%$ chance of memory. Participants knew that a recognition test would follow encoding but were told only to focus on remembering words cued as TBR and that TBF words were irrelevant.

Encoding. Prior to the experimental trials, eight neutral practice trials were provided. ${ }^{6}$ After the practice trials, 66 trials were completed including three neutral buffer trials at the beginning, three buffers at the end, and 60 experimental trials. Trials were presented in a pseudorandomized order such that no more than three items from each of the valence or cue conditions occurred in a row. Trials began with a fixation cross in the centre of the screen for $1000 \mathrm{~ms}$, followed by a word for study for $3000 \mathrm{~ms}$. An inter-stimulus interval (ISI) came after each word for $1500 \mathrm{~ms}$ after which the cue "RRRR" or "FFFF" appeared for $1000 \mathrm{~ms}$. Following the cue, participants provided their JOL. A scale appeared on the screen that read "How likely will you

\footnotetext{
${ }^{6}$ The number of practice trials was increased in this experiment relative to the others as participants were required to make speeded JOLs on each trial in addition to studying the items. This is in contrast to the other experiments where participants were only required to study each item and to follow the memory cue instruction. Considering this added difficulty, more practice was provided to ensure that participants understood the task and could perform at their best.
} 
be to remember or forget this item?" Below this text, a scale appeared ranging from 1 (will forget) to 10 (will remember). Four seconds were provided for participants to indicate their JOL response, which was made by pressing the corresponding number key at the top of the keyboard (the ' 0 ' on the keyboard was relabeled as ' 10 '). If a response was detected before the $4000 \mathrm{~ms}$, the trial ended and proceeded to an ISI for $500 \mathrm{~ms}$, otherwise the JOL scale remained on the screen for the entire $4000 \mathrm{~ms}$.

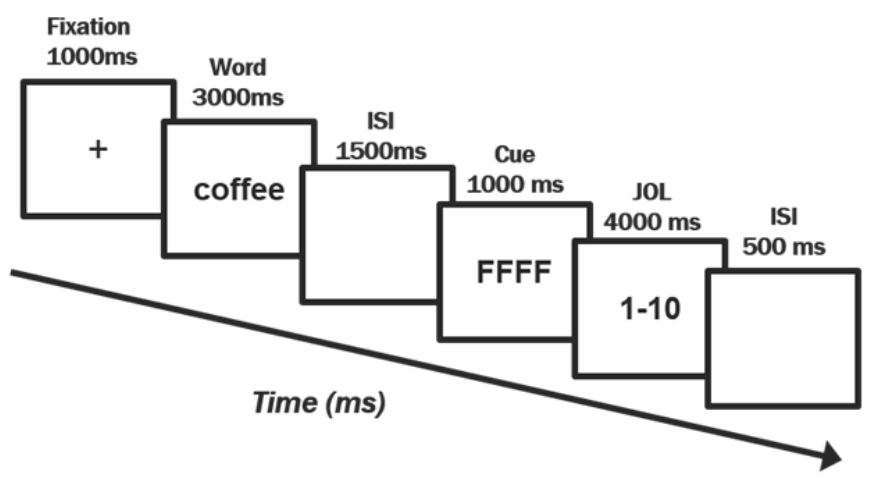

Figure 2. Sample TBF trial from the encoding phase of Experiment 1.

Filler task. Participants completed the DSST for two minutes.

Recognition. Recognition followed the same procedure as that outlined in Gallant and Yang (2014). Participants were presented with 60 old words randomly intermixed with 60 new words for a total of 120 trials. Instructions were to indicate whether each word was TBR, TBF, or new by pressing designated keys on the keyboard (' $\mathrm{z}$ ' $=\mathrm{R}$, '. ' $=\mathrm{F}$, Spacebar $=$ New). Each trial began with a fixation cross in the centre of the screen for $1000 \mathrm{~ms}$ followed by a TBR, TBF, or new word. Recognition was self-paced such that the trial did not move on until a response was detected, after which an ISI appeared for $500 \mathrm{~ms}$. Like encoding, selection of trials was pseudorandom with no more than three words from each valence or cue combination occurring in a row. 
At the end of the session, all participants completed the battery of questionnaires outlined in the General Method section (pages 40-42), after which they were debriefed on the purpose of the study and compensated for their participation.

\section{Data Analysis}

To gauge metacognitive monitoring, participants' JOLs (ranging from 1 to 10) were averaged across each combination of the valence and cue conditions and submitted to a mixedmodel ANOVA with the factors being valence, cue, and age. Relative accuracy of JOLs was examined by calculating Goodman-Kruskal Gamma $(\gamma)$ correlations between item-level JOLs and subsequent recognition for each participant. In line with prior practice (e.g., Friedman \& Castel, 2011; Kuhlmann \& Touron, 2011; Tauber \& Dunlosky, 2012), one-sample t-tests were calculated to determine whether gammas differed reliably from zero.

With regard to item recognition and source monitoring performance, all relevant dependent variables (i.e., hits, false alarms, $A^{\prime}, B^{\prime}{ }_{D}$, CSIM scores, and source misattributions) were indexed according to procedures outlined in the General Method (page 43-45). These indices were analyzed in a series of 3 (valence) $\times 2($ cue $) \times 2$ (age) ANOVAs. The cue factor was removed from analyses where dependent variables could not be distinguished by cue.

\section{Results}

\section{Metacognitive Monitoring: $\mathrm{JOLs}^{7}$}

Across age and valence conditions, JOLs were higher for TBR item $(M=6.63, S D=$ 1.55) than for TBF items $(M=3.59, S D=1.83)$ as revealed by a main effect of cue, $F(1,68)=$ 127.87, $p<.001, \eta_{p}^{2}=.65$. Overall monitoring was also influenced by valence, with lower JOLs for neutral $(M=4.83, S D=1.22)$ relative to positive $(M=5.32, S D=1.46 ; p<.001, d=0.71)$

\footnotetext{
${ }^{7}$ The data of one older adult were excluded from this analysis as inspection of their JOL data revealed that they withheld JOLs for over $50 \%$ of trials.
} 
and negative words $(M=5.20, S D=1.30 ; p<.001, d=0.59), F(2,136)=22.94, p<.001, \eta_{p}^{2}=$ .25 (see Figure 3); there was no difference between positive and negative items. No other effects emerged, Fs $<1.78$, ps $>.17$.

Given that JOLs were sensitive to cue and valence, gammas were calculated between JOLs and recognition for each cue and valence condition. Both age groups' gammas for TBR items differed from zero, suggesting that relative accuracy of JOLs was above chance (older $\gamma=$ $.18, S D=.51 ; p<.05 ;$ young $\gamma=.16, S D=.44 ; p<.05)$; however, gammas for TBF items did not differ from zero in either group $(\gamma \mathrm{s}<.11, p s>.10)$. With regard to valence, older adults' gammas for positive $(\gamma=.31, S D=.54 ; p<.01)$ and negative items $(\gamma=.18, S D=.46 ; p<.05)$ differed from zero, whereas neutral gammas did not $(\gamma=.06, p=.24)$. Young adults' gammas differed from zero for positive $(\gamma=.20, S D=.49 ; p<.05)$, negative $(\gamma=.19, S D=.56 ; p<.05)$, and neutral items $(\gamma=.20, S D=.43 ; p<.01)$. No age differences in gammas emerged $(p s>.21)$.

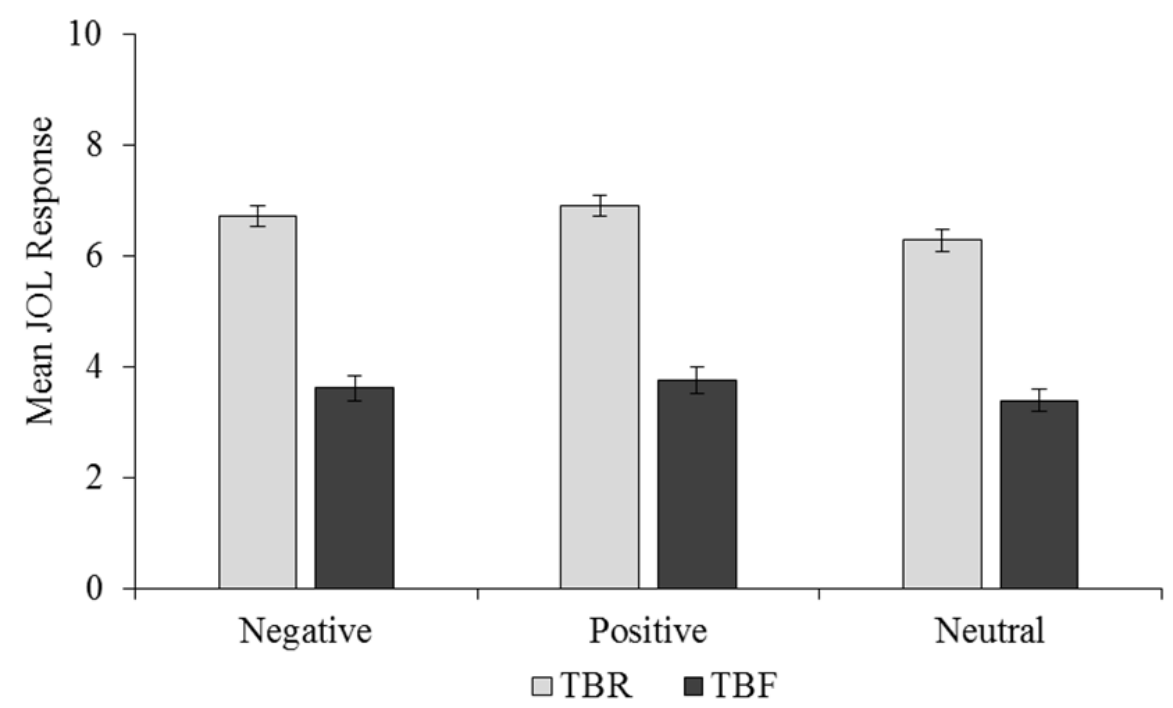

Figure 3. Mean JOL responses in Experiment 1 as a function of valence and cue, collapsed across age groups. Error bars represent standard error of the mean.

Together, these analyses imply age equivalence in prospective monitoring because no age differences were observed in either JOLs or their relative accuracy. Results also show that JOLs 
were higher for TBR than for TBF words as well as for emotional relative to neutral words, suggesting that both age groups' predictions were sensitive to memory cues and emotion.

\section{Item Recognition Performance}

The hits, false alarms, discrimination accuracy $\left(A^{\prime}\right)$, and response bias $\left(B^{\prime}{ }_{D}\right)$ scores for young and older adults are presented in Table 3.

Table 3

Means and Standard Deviations for Item Recognition Indices in Experiment 1

\begin{tabular}{lccccccc}
\hline & \multicolumn{3}{c}{ Young Adults } & & \multicolumn{3}{c}{ Older Adults } \\
\cline { 2 - 5 } \cline { 7 - 8 } & Negative & Positive & Neutral & & Negative & Positive & Neutral \\
\hline TBR Hits & $.88(.11)$ & $.83(.16)$ & $.79(.19)$ & & $.83(.15)$ & $.87(.14)$ & $.79(.15)$ \\
TBF Hits & $.78(.21)$ & $.80(.18)$ & $.73(.19)$ & & $.76(.17)$ & $.77(.21)$ & $.75(.18)$ \\
False Alarms & $.24(.19)$ & $.21(.19)$ & $.16(.12)$ & & $.33(.21)$ & $.40(.20)$ & $.28(.21)$ \\
$A$ & $.87(.10)$ & $.87(.09)$ & $.88(.06)$ & & $.82(.08)$ & $.80(.10)$ & $.83(.11)$ \\
$B{ }_{D}$ & $-.13(.71)$ & $-.12(.59)$ & $.18(.62)$ & & $-.23(.62)$ & $-.44(.54)$ & $-.03(.62)$ \\
\hline
\end{tabular}

Note. Standard deviations are presented in parentheses.

The 3 (valence) $\times 2($ cue $) \times 2$ (age) ANOVA on hit rates revealed greater overall recognition of $\operatorname{TBR}(M=.83, S D=.11)$ relative to $\mathrm{TBF}$ items $(M=.77, S D=.16), F(1,69)=$ $22.26, p<.001, \eta_{p}^{2}=.24$. As well, hit rates were modulated by valence, $F(2,138)=7.20, p<$ $.01, \eta_{p}^{2}=.10$, with reduced recognition of neutral $(M=.77, S D=.15)$ relative to positive $(M=$ $.82, S D=.14 ; p<.001, d=0.34)$ and negative items $(M=.81, S D=.14 ; p<.001, d=0.47)$; positive and negative items did not differ $(p=.72)$. No other effects emerged, $F s<1.19, p>.10$.

A separate 3 (valence) $\times 2$ (age) ANOVA on false alarm rates showed that older adults $(M=.34, S D=.19)$ had an increased tendency to identify new items as old compared to young adults $(M=.20, S D=.13), F(1,69)=12.20, p<.01, \eta_{p}^{2}=.15$. False alarm rates also varied as a function of valence, $F(2,138)=14.93, p<.001, \eta_{p}^{2}=.18$, which interacted with age, $F(2,138)$ 
$=4.74, p<.05, \eta_{p}^{2}=.06$. Planned contrasts revealed greater false alarm rates for positive compared to negative items $(p<.01, d=0.51)$ and neutral words $(p<.01, d=0.76)$ in older adults; their false alarms to negative items were marginally higher than neutral items $(p=.07, d$ $=0.32$ ). In contrast, when compared to neutral items, young adults' false alarm rates were elevated for both positive $(p<.01, d=0.44)$ and negative items $(p<.01, d=0.64$; see Figure 4$)$.

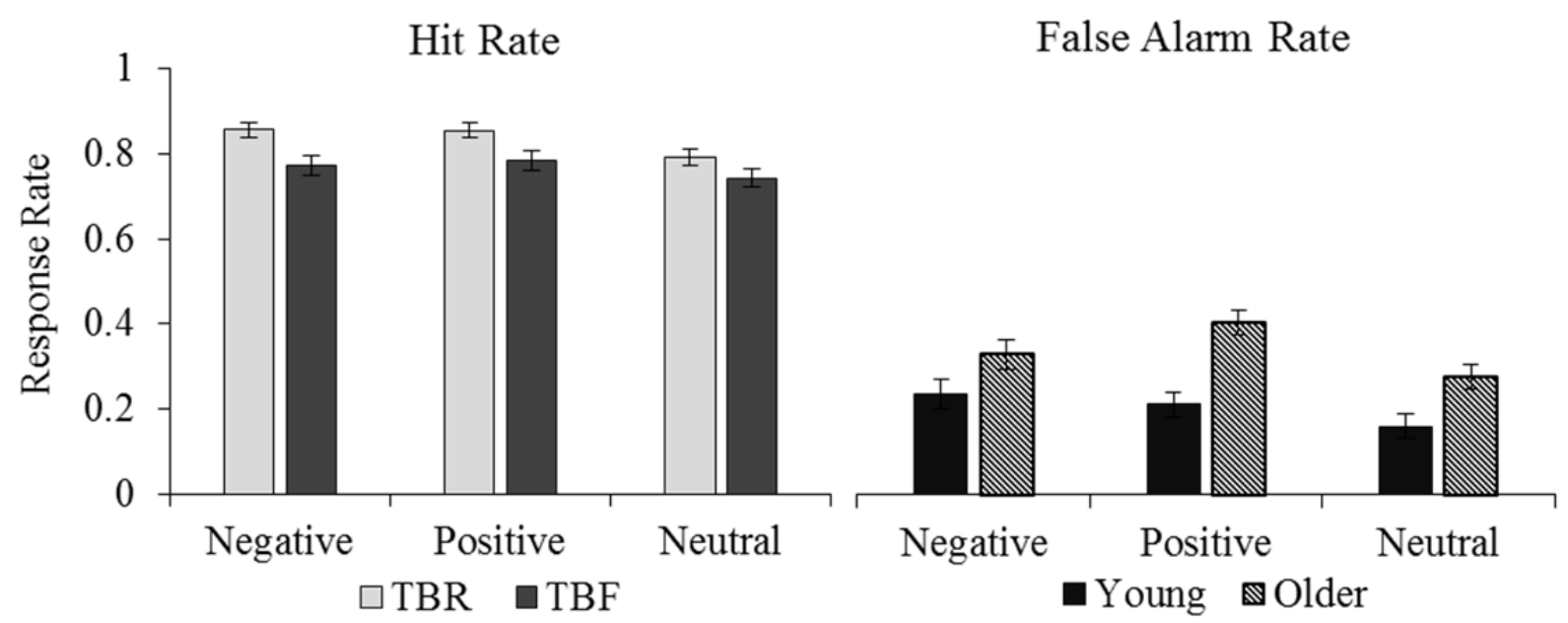

Figure 4. Proportions of hits and false alarms in Experiment 1. Error bars represent standard error of the mean.

Concerning signal detection indices, the ANOVA on discrimination accuracy $\left(A^{\prime}\right)$ showed reduced discrimination in older $(M=.82, S D=.08)$ relative to young adults $(M=.88$, $S D=.07), F(1,69)=10.30, p<.01, \eta_{p}^{2}=.13$. No other effects were observed, $F s<1.11, p s>$ .33. The 3 (valence) $\times 2$ (age) ANOVA on response bias revealed that $B{ }_{D}$ scores varied by valence, $F(2,138)=20.02, p<.001, \eta_{p}^{2}=.23$. Relative to response bias scores for neutral items $(M=.07, S D=.62)$, scores fell below zero for both negative $(M=-.18, S D=.66 ; p<.001, d=$ $0.53)$ and positive items $(M=-.28, S D=.59 ; p<.001, d=0.75)$, the latter two of which did not differ $(p=.10)$. All other effects were nonsignificant, Fs $<2.53, p>.12$. One-sample $t$-tests against zero confirmed liberal response biases for positive and negative items as they both 
differed reliably from zero in the negative direction $(t s>-2.24$, $p s<.05)$. Response bias for neutral items, in contrast, did not differ from zero $(p=.32)$.

To summarize, greater hit rates for TBR relative to TBF items illustrated the directed forgetting effect, independent of age or valence. Both hit and false alarm rates were also higher for emotional relative to neutral items but discrimination accuracy analyses suggested that emotion did not influence participants' ability to discriminate between old and new items.

Discrimination accuracy was, however, reduced for older adults, suggesting that they were less able than young adults to discriminate between old and new items. This age effect is likely tied to older adults' increased false alarm rate as no age differences were observed in hit rates.

Finally, response bias analyses implied that participants adopted a more liberal bias to respond 'old' to emotional items whereas no indication of a bias emerged for neutral items.

\section{Source Monitoring Performance}

Table 4 displays mean source monitoring performance including average CSIM scores and misattributions of new items to sources across age groups, cues, and valence conditions. Table 4

Means and Standard Deviations for Source Monitoring Indices in Experiment 1

\begin{tabular}{|c|c|c|c|c|c|c|}
\hline & \multicolumn{3}{|c|}{ Young Adults } & \multicolumn{3}{|c|}{ Older Adults } \\
\hline & Negative & Positive & Neutral & Negative & Positive & Neutral \\
\hline \multicolumn{7}{|l|}{ Correct Attributions ${ }^{\text {a }}$} \\
\hline TBR items as TBR & $.63(.19)$ & $.69(.23)$ & $.65(.20)$ & $.55(.26)$ & $.80(.18)$ & $.64(.22)$ \\
\hline TBF items as TBF & $.67(.25)$ & $.64(.25)$ & $.66(.23)$ & $.58(.25)$ & $.32(.26)$ & $.51(.22)$ \\
\hline \multicolumn{7}{|l|}{ Source False alarms ${ }^{b}$} \\
\hline New items as TBR & $.05(.08)$ & $.07(.09)$ & $.04(.09)$ & $.09(.08)$ & $.25(.17)$ & $.12(.10)$ \\
\hline New items as TBF & $.18(.16)$ & $.14(.09)$ & $.11(.09)$ & $.23(.19)$ & $.14(.09)$ & $.16(.15)$ \\
\hline
\end{tabular}


With respect to correct attributions, young adults showed higher source monitoring performance relative to older adults (young: $M=.66, S D=.10$ vs. older: $M=.57, S D=.09$ ), $F(1$, $69)=15.18, p<.001, \eta^{2}=.18$. There were also interactions of age and cue, $F(1,69)=10.58, p$ $<.01, \eta_{p}^{2}=.13$, and valence and cue, $F(1.66,114.36)=16.16, p<.001, \eta_{p}^{2}=.19$, which were qualified by a three-way interaction of age, valence, and cue, $F(1.66,114.36)=8.45, p<.01, \eta^{2}$ $=.11$. As illustrated in Figure 5, these interactions were primarily driven by a differential influence of valence on source monitoring of TBR and TBF items in older adults, $F(1.60,54.54)$ $=21.34, p<.001, \eta_{p}^{2}=.39$, that was not present in young adults $(p=.49)$.

To unpack this interaction, planned comparisons were conducted within and between age groups. Relative to young adults, older adults showed enhanced source recognition of positive TBR items $(p<.05, d=0.55)$ and reduced source recognition of positive TBF items $(p<.001, d$ $=1.29)$ and neutral TBF items $(p<.01, d=0.66)$; however, no other age differences were observed ( $p s>.14)$. Within the older group, CSIM scores were higher for positive TBR relative to positive TBF items $(p<.001, d=1.37)$ and marginally higher for neutral TBR than TBF items $(p=.05, d=0.33)$; negative TBR and TBF CSIMs did not differ $(p=.65)$. Source recognition of positive TBR scores was also higher than that of negative $(p<.001, d=0.74)$ and neutral TBR items $(p<.001, d=0.67)$; negative and neutral TBR CSIMs did not differ $(p=.66)$. The opposite pattern was observed for TBF CSIMs, such that older adults' source monitoring was reduced for positive TBF relative to negative $(p<.001, d=0.84)$ and neutral TBF items $(p<$ $.001, d=0.76)$; source monitoring of negative and neutral TBF items did not differ $(p=.87)$. Given that the CSIM score is dependent on the proportion of items that were misattributed to the alternative source, these data also indicate that older adults misattributed $68 \%$ of positive TBF items to TBR sources. No other effects or interactions were observed, $F<.29, p>.75$. 


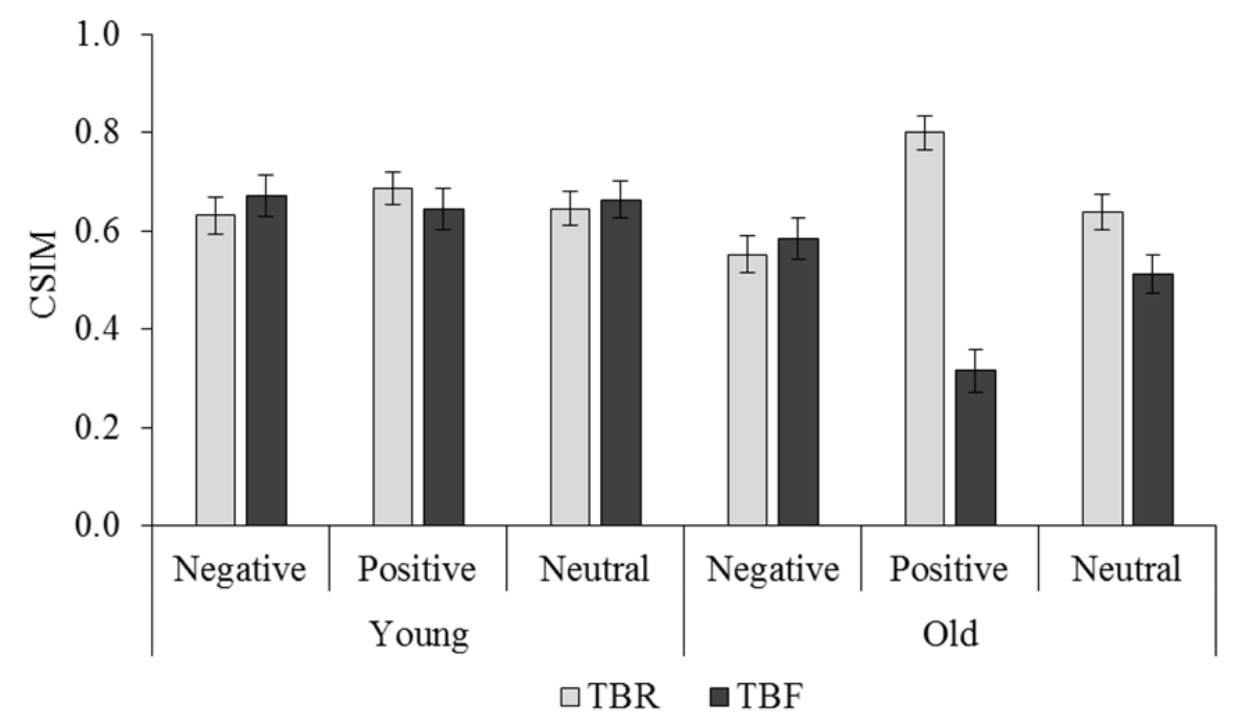

Figure 5. Proportions of CSIM scores in Experiment 1 displayed as a function of age, valence, and cue. Error bars represent standard error of the mean.

Last, proportions of misattributions of new items to TBR and TBF sources were submitted to an ANOVA with the factors being age, cue, and valence. Main effects emerged for all factors including age, $F(1,69)=12.20, p<.01, \eta_{p}^{2}=.15$, cue, $F(1,69)=22.38, p<.001, \eta^{2}{ }_{p}$ $=.25$, and valence, $F(2,138)=14.93, p<.001, \eta_{p}^{2}=.18$. In addition, the interaction of age and cue, $F(1,69)=6.83, p<.05, \eta_{p}^{2}=.09$, as well as that of valence and cue, $F(1.35,92.77)=$ $14.25, p<.001, \eta_{p}^{2}=.17$, were significant and qualified by a three-way interaction of these factors, $F(1.35,92.77)=6.90, p<.01, \eta^{2}=.09$. This three-way interaction was driven by a differential influence of valence on older adults' misattributions of new items to sources, $F(1.60$, $54.54)=21.34, p<.001, \eta_{p}^{2}=.39$, that was not seen in young adults $(p=.50$; Figure 6$)$. Planned comparisons revealed that, relative to young adults, older adults showed a higher tendency to misattribute positive $(p<.001, d=1.35)$ and neutral new items $(p<.001, d=0.90)$ to TBR sources; no other age differences were observed ( $p s>.08)$. Within the older group, the expected pattern of higher misattributions of new items to TBF relative to TBR sources was observed for 
negative $(p<.001, d=0.77)$ and neutral new items $(p<.05, d=0.27)$, whereas a propensity to misattribute positive new items to TBR than TBF sources emerged $(p<.01, d=0.42)$.

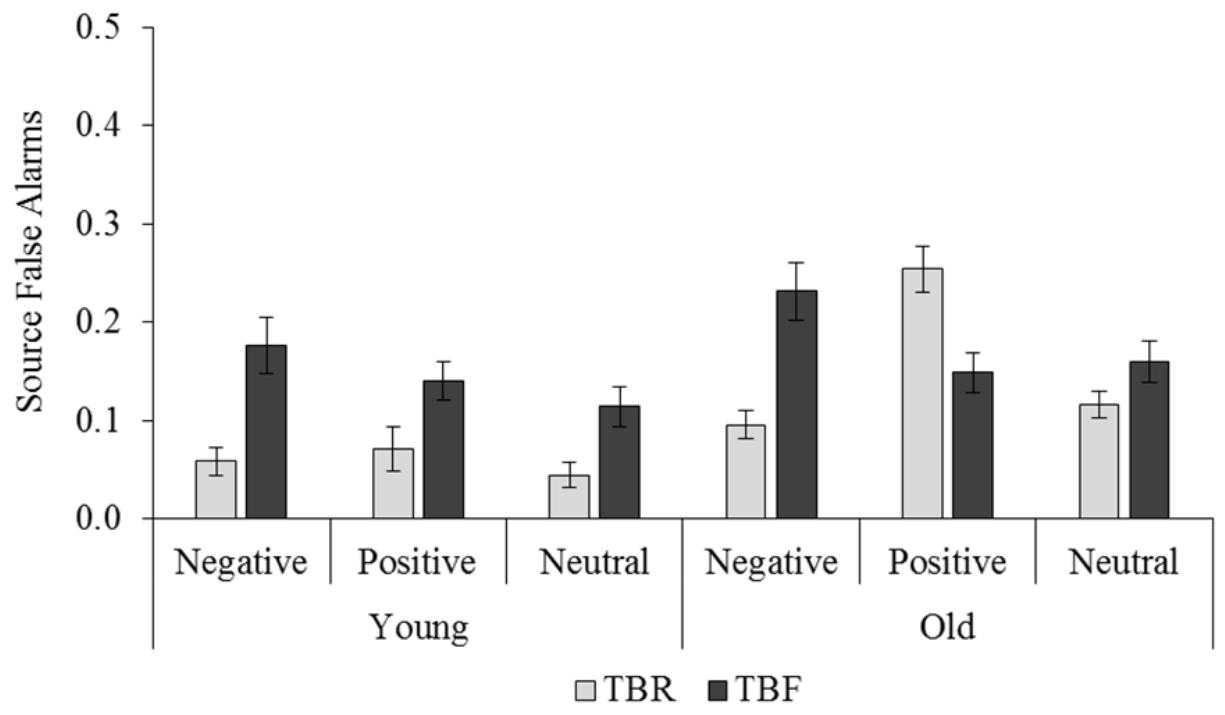

Figure 6. Proportions of misattributions of new items to sources in Experiment 1 displayed as a function of age, valence, and cue. Error bars represent standard error of the mean.

In sum, according to the source monitoring analysis, older adults showed a benefit in source memory for positive TBR items relative to positive TBF items. When making source guesses to new items, young adults were more likely to misattribute new items to TBF than TBR sources, regardless of valence, as expected. Older adults showed a similar pattern of source misattributions for negative and neutral new items; however, they were more likely to misattribute positive new items to TBR than TBF sources.

\section{Analysis of Individual Differences}

As seen in Table 2, there were several differences between the two groups on the cognitive and affective measures. As such, correlations were calculated within-groups to determine whether these individual differences were associated with the dependent variables that also showed age differences. No correlations were observed in the young sample, $r s>-.19$ and $<$ $-.02, p s>.28$. However, there was a positive correlation between older adults' performance on 
the DSST and their overall $A^{\prime}$ discrimination $(r=.36, p<.05)$ as well as their overall source recognition $(r=.37, p<.05)$. This suggests that higher processing speed, as inferred by DSST scores, may correspond to higher discrimination accuracy $\left(A^{\prime}\right)$ and source recognition in older adults. Mediation analyses were performed according to Hayes' technique (Hayes, 2012) to examine the possibility that the effects of age on discrimination accuracy were mediated by processing speed. When entering DSST scores as a mediator, the effect of age on $A^{\prime}$ 'scores attenuated and became marginally significant $(p=.06)$ whereas the effect of age on overall CSIM scores remained significant $(p<.05)$. This suggests that processing speed may have mediated the age differences observed on discrimination accuracy.

\section{Discussion}

Gallant and Yang (2014) suggested that, relative to young adults, valence differentially influences older adults' source monitoring of TBR and TBF items during recognition. While this prior study addressed questions concerning retrospective monitoring of information, the goal of the current experiment was to address the question of whether valence would similarly disrupt prospective monitoring of TBR and TBF items during encoding in older adults. In addition to testing hypotheses regarding monitoring performance, this experiment also afforded the opportunity to replicate our prior finding that both young and older adults can strategically control encoding of TBR and TBF emotional items (i.e., the directed forgetting effect).

Consider first performance on the directed forgetting task. The directed forgetting effect was observed across both age groups, even for emotional material. These results imply that both groups could strategically control encoding of TBR and TBF items that varied in valence. Importantly, this replicates not only our prior findings (Gallant \& Yang, 2014) but also other studies that have since examined the influence of emotion on cognitive control during aging via 
directed forgetting (Berger et al., 2016), think/no-think (Murray et al., 2011), and value-directed remembering tasks (Eich \& Castel, 2016). Thus, despite well-documented age differences in emotional memory (Reed \& Carstensen, 2012), these results again demonstrate that the ability to exert control over these memories is preserved with age.

Turning to monitoring performance, consistent with the literature (Hertzog \& Hultsch, 2000), JOLs were invariant across groups such that both young and older adults monitored differences between TBR and TBF sources during encoding, regardless of emotion. These cuerelated findings are consistent with Kuhlmann and Touron (2011), suggesting that both age groups can use extrinsic cues about memoranda to guide JOL monitoring. As well, both groups' JOLs were predictive of recognition for TBR items but not for TBF items, as indicated by above chance gamma correlations. These results fall in line with Friedman and Castel (2011), who similarly found relative accuracy of JOLs for TBR but not TBF items in young adults. To explain these results, the authors proposed that participants may have difficulty conceptualizing intentional forgetting. That is, we typically think about how likely we will be able to remember information (e.g., the time of an appointment) as opposed to how likely we will be able to forget the information in question. As a result, participants may have had difficulty predicting the degree to which they could successfully forget items. This does indeed seem to be the case as some overconfidence in the ability to forget was apparent when contrasting JOLs with subsequent recognition performance. Such overconfidence could be related to the use of immediate vs. delayed JOLs in the current study as individuals often overestimate their future memory performance when providing immediate JOLs as opposed to delayed JOLs (Nelson \& Dunlosky, 1991; Rhodes \& Tauber, 2011). Future research may consider using delayed judgments in the context of directed forgetting as they are thought to provide access to more 
diagnostic information from long-term memory. Nevertheless, despite participants'

overconfidence, their JOLs suggest some awareness of the dynamics of intentional forgetting and the ability to control information in memory.

Of primary interest, the monitoring of encoding data contrasted starkly with the source monitoring results. Whereas valence did not differentially influence JOLs for TBR and TBF items in either age group, older adults' source monitoring was better for TBR items than for TBF items, a difference that was not observed for negative and neutral items. In contrast, young adults' source monitoring did not vary by cue nor valence. Similar to Gallant and Yang (2014), it is possible that these results may be driven by a bias in older adults to misattribute positive items to TBR sources. This speculation is supported by the fact that older adults misattributed approximately $68 \%$ of positive TBF items to a TBR source compared to $36 \%$ for young adults. As well, when making source guesses, older adults showed a propensity to misattribute positive new items to TBR sources when the expected misattribution should be TBF (i.e., the source with low item memorability).

One possibility is that older adults' source misattributions reflect a tendency to attribute positive items to higher value sources (TBR) when memory for the actual source is lacking during recognition. Support for this speculation on differences in the perceived value of sources may be inferred from recent work examining the value of forgotten information (Castel et al., 2012; Rhodes, Witherby, Castel, \& Murayama, 2016). In an experiment by Castel and colleagues (2012), participants studied words paired with varying point values, of which they later recalled more high- than low-value words. However, on a subsequent cued-recall test for values associated with the words, participants attributed words that they had forgotten on the initial recall test to lower values and those that they had remembered to higher values. Based on these 
findings, the authors proposed a bias to deem forgotten information as less important or valuable. It is thus possible that older adults adopt a similar value-based theory about TBR and TBF sources, causing them to attribute higher-value (TBR) sources to positive items while avoiding low-value (TBF) sources. Of course, in the context of the current experiment, this hypothesis is only speculation and remains to be tested.

In summary, the findings provide evidence that prospective and retrospective monitoring processes are differentially influenced by valence, when sources differ on item memorability. Specifically, whereas older adults' ability to monitor encoding is unaffected by valence, retrospective monitoring of different sources appears to be disrupted by positive information. In addition, Experiment 1 provides novel findings that replicate and extend existing literature on cognitive control, metacognitive JOLs, and source monitoring. First, the results replicate the fact that young and older adults can reliably control the contents of memory even for emotional material (Berger et al., 2016; Eich \& Castel, 2016; Gallant \& Yang, 2014). Second, both age groups show metacognitive awareness of the dynamics of memory control, as indicated by the sensitivity of JOLs to directed forgetting cues. Consistent with prior findings (Friedman \& Castel, 2011; Kuhlmann \& Touron, 2011), these results suggest that the ability to rely on extrinsic cues (e.g., item memorability or cognitive operations; Koriat, 1997) when monitoring the acquisition of information from different sources is spared in older adults. This extends the literature on metacognition and aging showing that older adults not only are able to monitor the learning of TBR information but can adjust their monitoring when TBF information is involved. Finally, replicating prior findings from Gallant and Yang (2014), older adults' misattributions of sources to new items suggested a reduced ability to use knowledge of cognitive operations to inform their source guessing of positive information. 


\section{Chapter 6: Age Differences in Value-Based Directed Forgetting of Emotional Words Experiment 2}

The results of Experiment 1 as well as those of and Yang (2014) suggest that older adults have a source memory advantage for positive TBR words as compared to positive TBF words. When making source guesses in Experiment 1, older adults again showed a tendency to misattribute positive TBF and new items to TBR sources, while such a tendency was not observed for the other emotion conditions. This source misattribution bias suggests that older adults' ability to use knowledge of cognitive operations to guide source monitoring may be disrupted by positive information relative to young adults who show no difference in their source monitoring as a function of valence or cue. To account for this disruption in Experiment 1, I proposed that older adults may have an emotional goal-directed bias to assign higher-value sources and to avoid attributing lower-value sources to positive information. In other words, given their positivity bias (Mather \& Carstensen, 2005; Reed \& Carstensen, 2012), older adults may adopt a theory while completing the source monitoring task that "it's positive, so it is likely one I was supposed to remember and not forget.” The goal of Experiment 2 was to test this hypothesis by adopting a value-based cue manipulation in which TBR and TBF cues were replaced with cues that signaled a gain $(+10)$ or loss $(-10)$ of value.

This experiment also allowed us to address whether directed forgetting of emotional words observed in these studies and Gallant and Yang (2014) could extend beyond conceptual cues to remember and forget (i.e., 'RRRR' and 'FFFF'). Prior research using the value-directed remembering paradigm (Castel, 2007) has shown that young and older adults are comparable in their ability to strategically encode and retrieve high-value information (e.g., Castel, Farb, \& Craik, 2007) even for emotional material (Eich \& Castel, 2016). Castel (2007) has also suggested 
that the explicit presentation of value may be particularly motivating to older adults, enhancing their ability to selectively and strategically encode high- over low-value information. As such, it is possible that value-based cues may act as a more salient cue when directing participants to intentionally remember and forget. However, these studies have yet to address whether valuebased differences of information can be used to guide source monitoring for emotional material in young and older adults. Thus, by adopting a value-based cuing paradigm, the current experiment fills this gap in the literature by examining how value-based cues influence young and older adults' cognitive control and source monitoring of emotional information.

\section{Hypotheses}

Consistent with findings of Gallant and Yang (2014) and Experiment 1, I expected that both young and older adults would be able to control their encoding of positive and negative value words, regardless of emotional tone. If value-based cues do indeed act as a more salient cue to direct encoding (Castel, 2007), then I would expect young and older adults to show a similar magnitude of cognitive control over high- and low-value memories. Regarding source monitoring performance, if older adults' bias to attribute positive information to TBR sources is driven by differences in the perceived value of memory cues then they should show a similar tendency to attribute positive words to sources that signal a gain in value, regardless of the word's actual source.

\section{Method}

\section{Participants}

The final sample included 24 young adults (ages 18-25) and 24 older adults (ages 63-84). All participants completed the battery of assessments described in the General Method (pages 40-42). Consistent with the previous experiments, these data showed that older adults were more 
educated, had higher levels of positive affect, fewer symptoms of depression and anxiety, higher vocabulary, and slower psychomotor speed as indexed by the DSST. Characteristics of the final sample are displayed in Table 5.

Table 5

Characteristics of the Final Sample in Experiment 2

\begin{tabular}{|c|c|c|c|c|}
\hline & $\begin{array}{l}\text { Young Adults } \\
\quad(n=24)\end{array}$ & $\begin{array}{l}\text { Older Adults } \\
\quad(n=24)\end{array}$ & & \\
\hline Characteristic & $M(S D)$ & $M(S D)$ & $p$-value & $d$ \\
\hline Age in Years & $19.67(1.99)$ & $70.80(5.22)$ & $<.001 * *$ & -12.93 \\
\hline Male / Female Ratio & $2 / 22$ & $11 / 13$ & $.003 *$ & - \\
\hline Years of Education & $13.75(1.62)$ & $16.13(3.17)$ & $.002 *$ & -0.94 \\
\hline Positive Affect ${ }^{a}$ & $26.08(7.11)$ & $35.71(8.25)$ & $<.001 * *$ & -1.25 \\
\hline Negative Affect ${ }^{a}$ & $12.58(2.86)$ & $12.54(3.62)$ & .965 & 0.01 \\
\hline Depression $^{\mathrm{b}}$ & $6.75(5.56)$ & $3.83(4.96)$ & .061 & 0.55 \\
\hline Anxiety ${ }^{b}$ & $7.67(5.43)$ & $3.00(4.37)$ & $.002 *$ & 0.95 \\
\hline Shipley Vocabulary & $28.17(3.99)$ & $35.25(3.13)$ & $<.001 * *$ & -1.97 \\
\hline $\operatorname{DSST}^{\mathrm{c}}$ & $89.37(13.46)$ & $65.29(14.71)$ & $<.001 * *$ & 1.71 \\
\hline Short Blessed Test & - & $.33(.76)$ & - & - \\
\hline
\end{tabular}

Note. Independent-sample $t$-tests were computed for all between-subject comparisons apart from gender ratio, which was examined via Pearson's chi square. ${ }^{a}$ Measured with the Positive and Negative Affect Schedule; ${ }^{b}$ Measured with the DASS-21; ${ }^{c}$ Scores reflect number of correct solutions. DSST $=$ Digit Symbol Substitution Test. ${ }^{*} p<.05,{ }^{* *} p<.001$. $d=$ Cohen's $d$ for standardized effect size of the mean difference between groups.

\section{Materials}

The same list of 120 words as described in the General Method (page 38) was used in this experiment. The only difference was that instead of being paired with TBR or TBF cues, words in the current experiment were paired with high $(+10)$ or low $(-10)$ numbers, which signaled a gain or loss of value. 


\section{Procedure}

After providing informed consent, participants were seated in front of a laptop computer and provided with instructions on the encoding phase of the memory task.

Encoding. Participants were told they would need to learn a series of words, some of which would be designated as high-value items and others as low-value items. It was emphasized that participants should focus on remembering high-value words associated with +10 points while suppressing low-value words associated with -10 points. Like all other experiments, participants were told that their memory would be assessed on a subsequent recognition task, but that the focus should be on prioritizing the high-value items. Prior to beginning, three neutral practice trials were provided to familiarize participants with the task. If necessary, participants could repeat the practice trials up to three times. During the encoding phase, participants completed 66 trials in total, half of which were high-value and half of which were low-value. Six of these words were neutral buffer trials, three placed at the beginning and three at the end of the encoding list that were not tested during recognition. As in Figure 7, each trial began with a fixation cross directing participants' attention to the centre of the screen. This was followed by a word that was to be studied for $3000 \mathrm{~ms}$. An ISI followed the word for $1500 \mathrm{~ms}$, and then was replaced by a memory cue for $1000 \mathrm{~ms}$. Another ISI occurred for $500 \mathrm{~ms}$ before proceeding to the next trial. The experimental trials were presented in a pseudo-randomized fashion, such that no more than three trials of each valence or cue condition occurred in a row. 


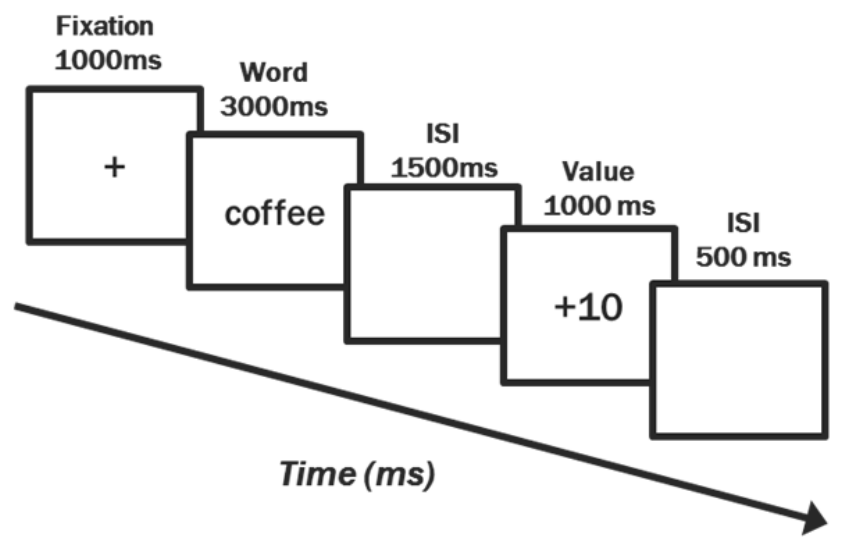

Figure 7. Sample of a high-value trial from the encoding phase of Experiment 2.

Filler task. Participants completed the DSST for two minutes.

Recognition. Following the filler task, a recognition task was administered. It included 120 trials, including the 60 high- and low-value items from encoding intermixed with 60 new items. On each trial, participants were required to determine whether the word was previously associated with +10 points, -10 points, or was new. Responses were recorded from the ' $\mathrm{z}$ ', '.', and spacebar keys that were respectively labelled as "+10", "-10", and "New". The 120 trials were presented in a pseudo-randomized order, with no more than three words from each cue or valence condition occurring in a row. Each trial began with a fixation cross at the centre of the screen for $1000 \mathrm{~ms}$, replaced by a word that remained on the screen until a response was detected. Following the response, an ISI occurred for $500 \mathrm{~ms}$ before proceeding to the next trial.

After the recognition task, participants completed the same battery of questionnaires described in the General Method (pages 40-42).

\section{Data Analysis}

Within item recognition and source monitoring analyses, dependent variables were defined according to the procedures outlined in the General Method (pages 43-45). These variables were analyzed in a series of 3 (valence) $\times 2$ (value: $+10,-10) \times 2$ (age) ANOVAs. As in 
Experiment 1, the value factor was removed from analyses where dependent variables could not be distinguished by value.

\section{Results}

\section{Item Recognition Performance}

Table 6 displays average recognition performance including hits, false alarms,

discrimination accuracy $\left(A^{\prime}\right)$, and response bias $\left(B^{\prime \prime}\right)$ scores within each condition across age groups.

Table 6

Means and Standard Deviations for Item Recognition Indices in Experiment 2

\begin{tabular}{lccccccc}
\hline & \multicolumn{3}{c}{ Young Adults $(n=24)$} & & \multicolumn{3}{c}{ Older Adults $(n=24)$} \\
\cline { 2 - 5 } \cline { 7 - 8 } & Negative & Positive & Neutral & & Negative & Positive & Neutral \\
\hline+10 Hits & $.82(.12)$ & $.79(.18)$ & $.73(.16)$ & & $.78(.17)$ & $.79(.18)$ & $.69(.21)$ \\
-10 Hits & $.62(.19)$ & $.61(.21)$ & $.48(.25)$ & & $.62(.21)$ & $.59(.26)$ & $.48(.25)$ \\
False Alarms & $.24(.22)$ & $.23(.18)$ & $.14(.13)$ & & $.28(.22)$ & $.29(.18)$ & $.18(.14)$ \\
$A{ }^{\prime}$ & $.82(.10)$ & $.82(.08)$ & $.83(.08)$ & & $.79(.12)$ & $.78(.12)$ & $.80(.09)$ \\
$B{ }_{D}$ & $.12(.65)$ & $.16(.61)$ & $.55(.45)$ & & $.06(.64)$ & $.00(.64)$ & $.44(.53)$ \\
\hline
\end{tabular}

Note. Standard deviations are presented in parentheses.

The 3 (valence) $\times 2$ (value) $\times 2$ (age) ANOVA on hits revealed better recognition of high-value $(M=.76, S D=.14)$ relative to low-value items $(M=.56, S D=.19), F(2,92)=93.26$, $p<.001, \eta_{p}^{2}=.67$. Valence also modulated hits, $F(2,92)=18.41, p<.001, \eta_{p}^{2}=.28$, such that neutral word recognition $(M=.59, S D=.19)$ fell below that of positive $(M=.70, S D=.17 ; p<$ $.001, d=0.72)$ and negative items $(M=.71, S D=.15 ; p<.001, d=0.88)$; the latter two conditions did not differ ( $p=.55$; Figure 8$)$. There were no other effects, $F s<1.09, p s>.34$.

A separate 3 (valence) $\times 2$ (age) ANOVA showed that false alarm rates to new items were similarly influenced by valence, $F(2,90)=22.87, p<.001, \eta_{p}^{2}=.33$, with reduced false 
alarms to neutral $(M=.16, S D=.13)$ relative to negative $(M=.26, S D=.22 ; p<.001, d=0.90)$ and positive items $(M=.27, S D=18 ; p<.001, d=1.18)$; positive and negative false alarms did not differ $(p=.69)$. No other effects emerged, Fs $<.91$, ps $>.62$.

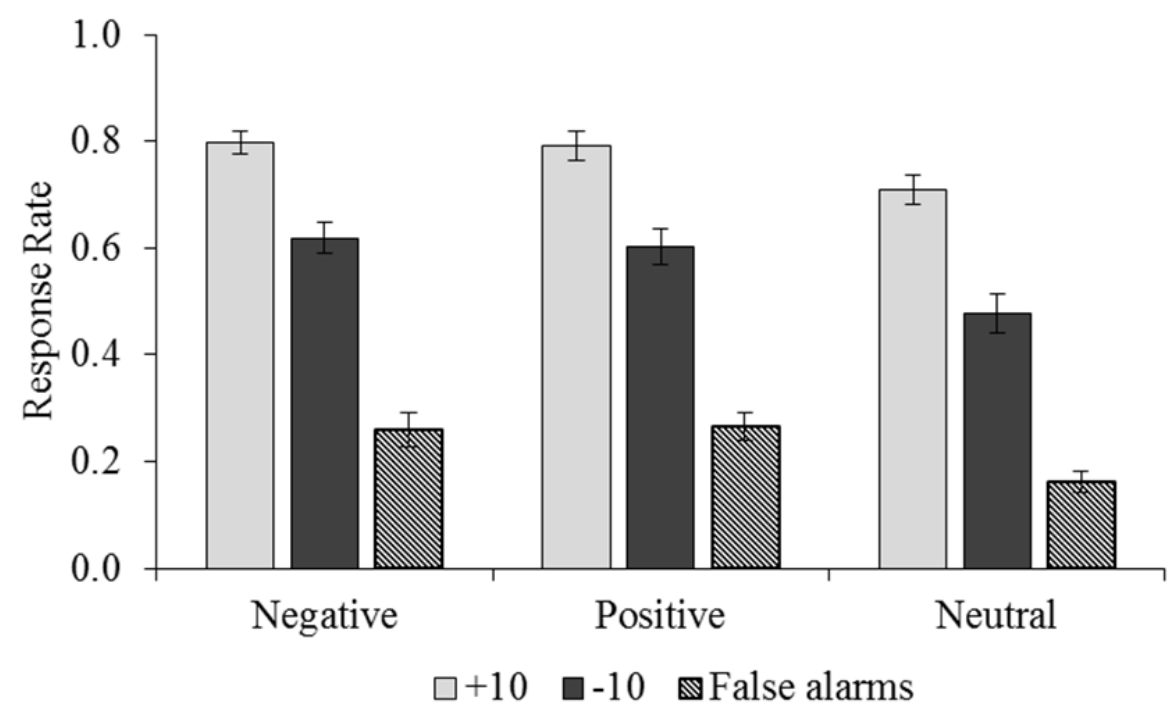

Figure 8. Proportions of hits and false alarms in Experiment 2 displayed as a function of valence and value. Error bars represent standard error of the mean.

The ANOVA on discrimination accuracy revealed that $A^{\prime}$ was not influenced by age or valence, $F s<1.99$, ps $>.17$. Analysis of response bias revealed a main effect of valence, $F(2$, $92)=37.12, p<.001, \eta_{p}^{2}=.45$, such that $B{ }_{D}$ scores were higher for neutral $(M=.49, S D .49)$ relative to negative $(M=.09, S D=.64 ; p<.001, d=1.02)$ and positive items $(M=.08, S D=$ $.63 ; p<.001, d=1.18)$; the latter two did not differ $(p=.82)$. One-sample $t$-tests against zero confirmed a conservative response bias for neutral items as scores were significantly greater than zero, $t(47)=6.99, p<.001$. In contrast, $B{ }_{D}$ scores for negative and positive items did not differ from zero ( $p s>.31)$. No other effects were observed, Fs $<.49$, ps $>.48$.

To summarize, the analysis on item recognition demonstrated greater memory for highvalue than low-value items, indicating that value manipulation was effective. Like Experiment 1, emotion enhanced participants' hit rate as well as their tendency to mistake new items as old 
(i.e., false alarms). Neither age nor valence influenced participants' ability to discriminate between old and new items. However, neutral items elicited a more conservative response bias in which both young and older adults required more memory evidence before accepting a word as old. In contrast, emotional items did not elicit a bias in either direction, suggesting that participants were equally likely to give old and new responses to emotional words.

\section{Source Monitoring Performance}

Table 7 displays source monitoring performance including average CSIM scores (i.e., correct attributions, see Page 45 for formula) and misattributions to new items (i.e., source false alarms) across older and young adults within each condition.

Table 7

Means and Standard Deviations for Source Monitoring Indices in Experiment 2

\begin{tabular}{|c|c|c|c|c|c|c|}
\hline & \multicolumn{3}{|c|}{ Young Adults } & \multicolumn{3}{|c|}{ Older Adults } \\
\hline & Negative & Positive & Neutral & Negative & Positive & Neutral \\
\hline \multicolumn{7}{|c|}{ Correct Attributions a } \\
\hline+10 items as +10 & $.70(.16)$ & $.72(.24)$ & $.77(.19)$ & $.62(.19)$ & $.75(.28)$ & $.79(.22)$ \\
\hline-10 items as -10 & $.71(.23)$ & $.68(.24)$ & $.74(.27)$ & $.73(.26)$ & $.45(.29)$ & $.66(.28)$ \\
\hline \multicolumn{7}{|c|}{ Source False Alarms ${ }^{\text {b }}$} \\
\hline New items as +10 & $.06(.08)$ & $.06(.09)$ & .03 (.04) & $.07(.09)$ & $.16(.12)$ & $.05(.08)$ \\
\hline New items as -10 & $.18(.16)$ & $.17(.13)$ & $.11(.11)$ & $.20(.18)$ & $.13(.15)$ & $.13(.11)$ \\
\hline
\end{tabular}

Note. Standard deviations are presented in parentheses; ${ }^{a}$ represents CSIM score according to the calculation on Page $45 ;{ }^{b}$ represents proportion of misattributions of new items to sources.

The 3 (valence) $\times 2$ (value) $\times 2$ (age) ANOVA on CSIM scores revealed a main effect of valence, $F(2,92)=5.54, p<.01, \eta_{p}^{2}=.11$, driven by greater overall source monitoring of neutral $(M=.72, S D=.18)$ relative to positive $(M=.66, S D=.16 ; p<.01, d=0.40)$ and negative items $(M=.68, S D=.14 ; p=.06, d=0.28)$, although this latter difference was marginal and so should be interpreted with caution. There was no difference between positive 
and negative CSIM scores $(p=.31)$. There was also an interaction of valence and cue, $F(2,92)=$ $9.54, p<.001, \eta_{p}^{2}=.18$, qualified by a three-way interaction between age, cue, and valence, $F(2$, $92)=5.81, p<.01, \eta_{p}^{2}=.12$. As illustrated by Figure 9 and planned follow-up analyses, this was driven by an interaction of valence and cue in older adults, $F(2,48)=13.17, p<.001, \eta_{p}^{2}=$ .39 , but not young adults $(p=.74)$. When comparing between age groups, older adults relative to young adults showed reduced CSIMs specifically for low-value positive items $(p<.01, d=$ 0.85 ); no other age differences were observed across groups ( $p s>.25)$. Within the older group, older adults' high-value CSIM scores were elevated for positive relative to negative items ( $p<$ $.05, d=0.44)$, while neutral and positive items did not differ $(p=0.57)$. Older adults' high-value CSIMs for negative items were also lower than those of neutral items $(p<.05, d=0.52)$. For low-value items, older adults' CSIM scores were reduced for positive relative to negative ( $p<$ $.001, d=1.01)$ and neutral items $(p<.01, d=0.65)$ while low-value CSIMs to negative and neutral items did not differ $(p=.18)$. Cue differences were also observed within each valence condition such that older adults' CSIM scores were greater for high-value than for low-value positive items $(p<.01, d=.72)$; no such differences were observed for negative or neutral items (ps $>.15$ ). Given that the CSIM score is dependent on the proportion of items that were misattributed the alternative source, these data also indicate that older adults misattributed 55\% of low-value positive items to a high-value source compared to $32 \%$ in young adults. No other effects emerged, Fs $<2.19$, ps $>.15$. 


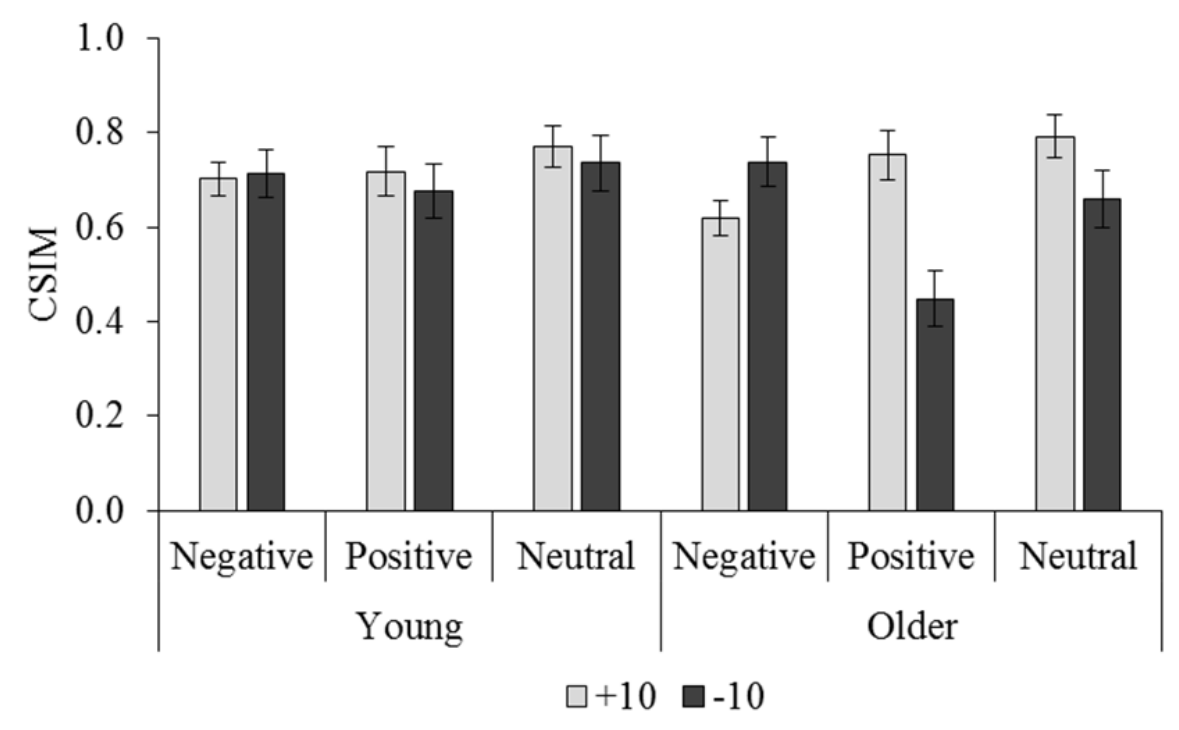

Figure 9. Proportions of CSIM scores in Experiment 2 displayed as a function of age, valence, and value. Error bars represent standard error of the mean.

Finally, misattributions of new items to sources were analyzed. The 3 (valence) $\times 2$ (value) $\times 2$ (age) ANOVA revealed main effects of valence, $F(1.74,80.10)=22.87, p<.001, \eta_{p}^{2}$ $=.33$, and cue, $F(1,46)=22.90, p<.001, \eta_{p}^{2}=.33$, as well as an interaction of these two factors, $F(2,92)=22.87, p<.01, \eta_{p}^{2}=.12$. This two-way interaction was further qualified by an interaction between age, valence, and cue, $F(2,92)=5.97, p<.01, \eta_{p}^{2}=.12$. In unpacking this interaction, it was found that only older adults displayed an interaction of valence and cue, $F(2$, $46)=7.57, p<.01, \eta_{p}^{2}=.25$; young adults' source false alarms did not vary as a function of valence or cue $(p=.15)$. As illustrated in Figure 10, young adults were more likely to attribute new items to low- than high-value sources for all three emotion conditions. Older adults showed a similar pattern for negative $(p<.01, d=0.77)$ and neutral $(p<.01, d=0.65)$ words; however, there was no such distinction for positive items $(p=.53)$. Rather, planned comparisons showed that older adults assigned a higher proportion of positive new items to high-value sources relative 
to negative $(p<.01, d=0.74)$ and neutral $(p<.01, d=1.39)$ new items. No other effects were observed in the analysis, Fs $<1.59$, ps $>.21$.

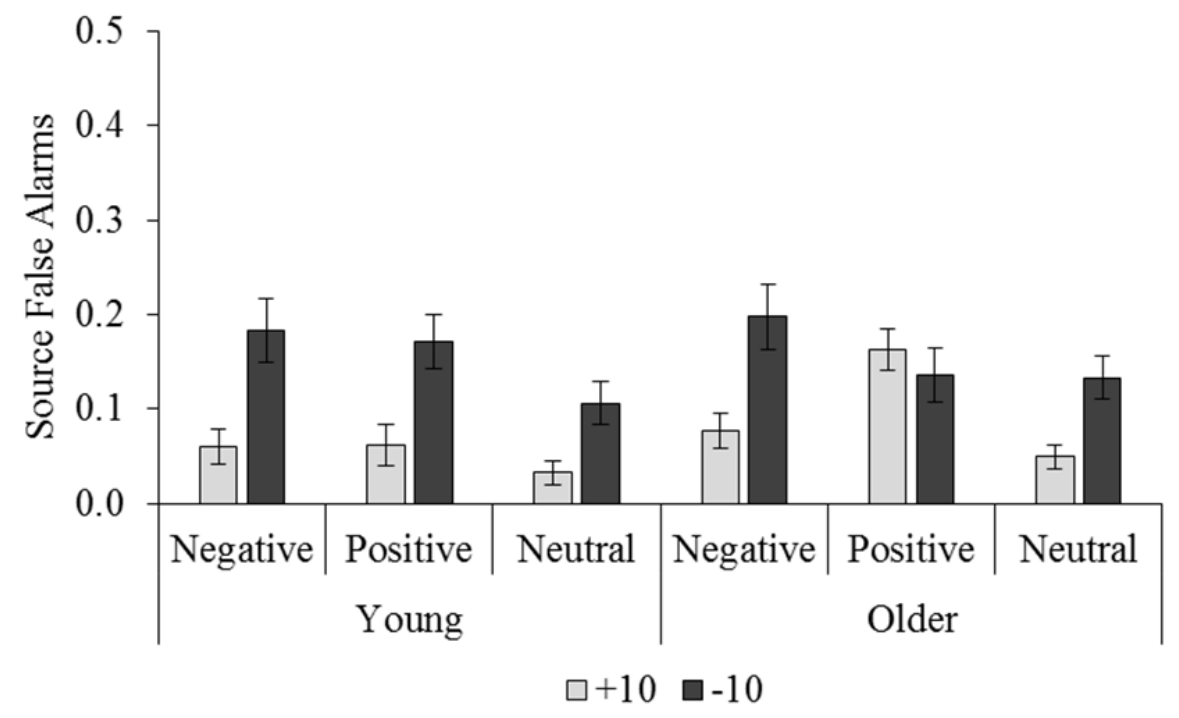

Figure 10. Proportions of misattributions of new items to sources in Experiment 2 displayed as a function of age, valence, and value. Error bars represent standard error of the mean.

To summarize, these analyses revealed a source memory advantage for positive items that were high in value relative to those that were low in value for older adults. This contrasts with young adults whose source monitoring for high- and low-value items was not influenced by emotion. When making source guesses to new items, young adults showed the expected tendency to attribute new items to low-value sources. Older adults showed this tendency for negative and neutral new items, although they showed no differentiation between their source guesses to positive new items.

\section{Analysis of Individual Differences}

No correlations were observed between any of the variables with age differences in Table 5 and the dependent variables that also showed age differences $(r s>-.32$ and $<.38, p s>.07)$. 


\section{Discussion}

The purpose of this experiment was two-fold. First, I sought to test the hypothesis that the interaction of age and emotional valence on source attributions observed in Experiment 1 as well as in Gallant and Yang (2014) was driven by older adults' preference to attribute positive items to high-value sources than to low-value sources. As well, I examined whether the strategic control of emotional information previously observed (Gallant \& Yang, 2014) could be replicated when memory is cued by values that signal either a loss or gain in value as opposed to conceptual cues to remember or forget. This would provide further evidence that both age groups can strategically control memory for value-based information (e.g., Castel et al., 2007) while providing information on how these representations become organized and monitored within memory.

Regarding item memory performance, both young and older adults were similarly able to strategically control their encoding of high- and low-value information. This contrasts with Gallant and Yang (2014) and Experiment 1, in which older adults showed a reduced directed forgetting effect relative to young adults. However, these results are consistent with findings in the value-directed remembering literature, in which young and older adults show similar rates of selectivity in their memory for high- and low-value items (e.g., Castel et al., 2007; Eich \& Castel, 2016). These consistencies with the value-directed remembering literature may suggest that older adults are more efficient at utilizing explicit value-based cues to direct cognitive resources toward relevant information as compared to more conceptual cues to remember or forget. Importantly, age equivalence in value-directed forgetting occurred even for emotional information, replicating previous experiments that have found no age differences in control of 
emotion in the context of item-directed forgetting (Berger et al., 2017; Gallant \& Yang, 2014), think/no-think (Murray et al., 2011), or value-directed remembering tasks (Eich \& Castel, 2016).

When examining source monitoring, young adults' performance was not modulated by value or valence, replicating our previous experiments (Gallant \& Yang, 2014, and Experiment 1). In contrast, older adults showed elevated source memory for positive words that were high relative to low in value whereas their source memory for negative and neutral words did not vary by cue. When making source guesses, a tendency to attribute new items to low-value relative to high-value sources was predicted, implying a reliance on knowledge of cognitive operations about strongly encoded high-value items and weakly encoded low-value items. This pattern of findings was indeed observed for young adults, regardless of emotion. Older adults showed a similar source guessing tendency for negative and neutral new items but not for positive new items. Instead, they were more likely to misattribute positive TBF and new items to high-value sources. Like Experiment 1, this pattern of findings may imply that positive words disrupt older adults' ability to use knowledge of cognitive operations during source monitoring, particularly when sources differ in their value or relevance for later memory (Gallant \& Yang, 2014).

One possible explanation for these results is that older adults base their source decisions for positive items not on cognitive operations but on their own beliefs about what memories from a certain source should be like (Johnson et al., 1993; Mitchell \& Johnson, 2009). This is consistent with the "it had to be you effect" that has been described within the source monitoring framework (Johnson et al., 1993). For example, when participants monitor words generated by themselves (internal) versus the experimenter (external), they are more likely to misattribute new items as coming from the external source when not sure about their memory (i.e., "it had to be you, not me"). As previously proposed, older adults may therefore base their source decisions on 
beliefs about positive information being higher in value than negative or neutral information, driven by their age-related positivity bias (Mather \& Carstensen, 2005). This is further supported by the fact that the source monitoring bias in the current experiment was isolated to positive words whereas monitoring of negative and neutral words was not influenced by the cue. As described by Kuhlmann and Bayen (2016), this response tendency in older adults may reflect a strategy or form of compensation when memory for the actual source of information is absent.

Although this pattern of results may suggest that older adults' bias to attribute positive items to higher-value sources is specific to retrieval, it remains possible that mechanisms operating at encoding could also contribute to this effect. For instance, it could be argued that older adults' attention is captured by positive words during encoding (prior to the cue) and that the cognitive operations associated with processing positive words are greater than those operations associated with the cue. As a result, older adults may rely more on the operations associated with positive words as opposed to those associated with the cue during source monitoring, creating a tendency to misattribute TBF and new items to TBR sources. However, based on the first two experiments, conclusions cannot be drawn about how attention-based processes were influenced during encoding. In the following experiment, the ERP technique was adopted to address this question as well as to examine age differences in the neural correlates of directed forgetting. 


\section{Chapter 7: Age Differences in the ERP Correlates of Emotional Directed Forgetting Experiment 3}

The results of Gallant and Yang (2014) as well as Experiments 1 and 2 in this dissertation suggest that young and older adults can control the encoding of TBR and TBF information, as indexed by the directed forgetting effect. Of primary interest to this dissertation was the replication that participants could elicit control over both negative and positive stimuli, despite a wealth of evidence for the persistent effects of emotion in memory (Labar \& Cabeza, 2006) and an age-related bias to remember information high in positive valence in older adults (Mather \& Carstensen, 2005). What remains to be addressed in Experiment 3 is whether young and older adults differ in the neural mechanisms that support directed forgetting of emotion.

Examining the effects of age on neural activity is important as age differences in taskrelated brain activity are often observed, even when behaviour is similar between young and older adults (Cabeza \& Dennis, 2012; Friedman, 2003, 2012; Grady, 2012;). This is not surprising given the changes that the brain undergoes as it ages, including (but not limited to) declines in the volume and function of the PFC, loss of integrity in white matter tracts, and alterations in the level of neurochemicals (e.g., serotonin, noradrenaline) that circulate the brain (for a review, see Hedden \& Gabrieli, 2004). In terms of task-related activity, an intriguing finding has been that aging can have opposing effects on brain activity. As reviewed by Grady (2012), older adults sometimes show reductions in activity relative to young adults, reductions that are thought to be associated with age-related deficits in cognitive processing. At other times, older adults show increased brain activity during a cognitive task when compared to young adults (e.g., Davis et al., 2008; Grady et al., 1994; Rizio \& Dennis, 2014). For instance, in the 
ERP literature, older adults often show an anterior shift in the topography of the $\mathrm{P} 300$ (or $\mathrm{P} 3 \mathrm{~b}^{8}$ ), a positive-going ERP component with a parietal (or posterior) scalp distribution that is thought to underlie goal-directed attention (Friedman, 2003, 2012; O'Connell et al., 2012). This age-related anterior shift in activity has been argued to reflect functional compensation whereby higher-order cognitive resources associated with the PFC are recruited to mitigate age-related deficits in other brain regions (Dennis \& Cabeza, 2008; Grady, 2012). Whether such age-related increases in brain activity are deemed compensatory depends on the degree of correspondence between this activity and task-related performance. According to Cabeza and Dennis (2012), in defining successful compensation, older adults' increased frontal activity should positively correlate with cognitive performance, implying a functional relationship. It has also been argued that this brainbehaviour relationship should be considered compensatory if it occurs in older and not young adults, suggesting that the pattern of increased activity contributes to performance in an agespecific manner (Grady, 2012).

In the context of directed forgetting, to the best of my knowledge, only one study has examined age differences in the neural correlates underlying directed forgetting using fMRI (Rizzio \& Dennis, 2014). As described in Chapter 2, older adults in this study showed increased reliance on the PFC in the right inferior and left middle frontal gyri during attempts to encode TBR items, despite the finding of age equivalence in the behavioural directed forgetting effect. Consistent with the notion of 'successful compensation' (Cabeza \& Dennis, 2012), the authors found a positive association between older adults' PFC activity and successful recognition of TBR items, implying a functional role of this increase in task-related brain activity. These

\footnotetext{
${ }^{8}$ The P3 component encompasses two distinct subcomponents, the P3a and P3b. The P3a is typically evoked around $250 \mathrm{~ms}$ after stimulus onset over anterior sites and is thought to reflect an orienting response to task-irrelevant stimuli (Friedman, 2012; O'Connell et al., 2012). The P3b has more of a parietal distribution and onsets slightly later than the P3a, around 300-600 ms following stimulus onset. In contrast to the P3a, the P3b is argued to reflect top-down allocation of attention toward task-relevant stimuli.
} 
findings suggest that while the directed forgetting effect may not differ across groups, the neural processes contributing to the effect do indeed change with age. Although informative, the results of this study cannot shed light on the time course of the age-related effects associated with directed forgetting or how they might be modulated by the emotional tone of information. To address this gap in the literature, the ERP technique was adopted in Experiment 3 due to its high temporal resolution, allowing for neural activity to be tracked on a millisecond basis.

To provide some background, ERPs are derived from scalp-recorded EEG and represent voltage fluctuations arising from sensory, motor, or cognitive processes time-locked to a specific event or stimulus (Pires, Leitão, Guerrini, \& Simões, 2014). They index the summation of activity from large numbers of postsynaptic potentials that are primarily cortical. These voltage fluctuations are illustrated in the ERP waveform as a series of peaks and troughs that can be either negative or positive in amplitude. When using ERPs to study neural processes, it is not the peaks observed in the waveform that are of interest, but the underlying ERP components that sum together to create these peaks (Luck, 2014). As defined by Luck, ERP components are a set of scalp-recorded voltage changes reflective of specific neural or psychological processes that vary systematically across certain conditions and individuals. For example, modulation to the parietal P3b component that peaks around $300 \mathrm{~ms}$ following a stimulus is thought to represent top-down allocation of attention and is sometimes predictive of later memory (Luck, 2014; Wilding \& Ranganath, 2012). ERP components are often divided according to exogenous sensory components that are automatically elicited by the presence of a stimulus and more controlled endogenous components that reflect task-dependent brain processes (Luck, 2014). Through analysis of ERP components in different segments of the waveform, we can better examine how sensory and higher-order cognitive processes unfold over time. 
Several studies have used the ERP technique to elucidate the mechanisms of directed forgetting in young adults, suggesting distinct ERP correlates in relation to TBR and TBF cues (e.g., Brandt et al., 2013; Gallant \& Dyson, 2016; Hauswald et al., 2010; Paller, 1990; PazCaballero \& Menor, 1999; Paz-Caballero et al. 2004; van Hooff \& Ford, 2011; Yang et al., 2012). For instance, enhanced positive potentials over frontal sites are found to emerge early in the time window (100-300 ms) following a cue to forget and are sustained into later epochs (> 300 ms; e.g., Brandt et al., 2013; Gallant \& Dyson, 2016; Hauswald et al., 2010; Paz-Caballero et al., 2004; van Hooff \& Ford, 2011). These frontal ERPs have been argued to reflect a blocking mechanism that continually suppresses TBF items until the next item is presented. On the other hand, TBR cues evoke a slow-going positivity over parietal scalp sites that occurs around 300$600 \mathrm{~ms}$ following cue onset (i.e., the P3b), and is thought to represent strategic allocation of attentional resources toward stimuli (Brandt et al., 2013; Gallant \& Dyson, 2016; Hauswald et al., 2010; Nieuwenhuis, Aston-Jones, \& Cohen, 2005; Paz-Cabellero et al., 2004). Together, these divergent patterns of brain activity lend support to an active account of intentional forgetting in which selective rehearsal mechanisms (parietal positivity) that enhance processing of TBR items and active processes (frontal positivity) that deprioritize or suppress TBF items (Anderson \& Hanslmayr, 2014).

Whether these cue-related ERP patterns are modulated by emotion has been examined primarily in young adults (e.g., Brandt et al., 2013; Hauswald et al., 2010; Gallant \& Dyson, 2016; Yang et al., 2012). In such studies, attention is paid to the late positive potential (LPP) as emotion typically enhances this component due to increased attention toward emotional salience (e.g., Brown, van Steenbergen, Band, de Rover, \& Nieuwenhuis, 2012; Hajcak \& Olvet, 2008; Schupp et al., 2000; for a review, see Olofsson, Nordin, Sequeira, \& Polich, 2008). For instance, 
Brandt and colleagues (2013) found enhanced LPP responses to emotional over neutral words even before presentation of the memory cue. This modulatory effect of emotion persisted after the cue, with enhanced responses for negative words under TBR conditions. Frontal activity association with the TBF cue, on the other hand, was not influenced by emotion. Work that I later conducted along with my colleague further supported these findings, showing that emotional words at both high and low levels of arousal specifically modulate TBR- but not TBFrelated activity (Gallant \& Dyson, 2016). Together, these results suggest that emotional words may have a specific impact on the selective rehearsal mechanisms engaged during implementation of TBR cues but not frontal-based processes elicited during forgetting.

With regard to this above research, however, it is unknown whether older adults would show a similar modulation of ERPs during directed forgetting of emotion. Prior ERP research has shown that the LPP response to negative stimuli declines across the lifespan (Kisley, Wood, \& Burrows, 2007; Wood \& Kisley, 2006) and so it is reasonable to expect age differences in the LPP component elicited by emotional words and in the parietal positivity elicited by TBR cues following emotional words. As well, the parietal positivity typically elicited by TBR cues may be further modulated by age, regardless of emotion, given that older adults tend to show an anterior shift in the distribution of the P3b component (Friedman, 2003, 2012). The first goal of Experiment 3 is therefore to address how age and emotion differentially influence ERPs associated with attempts to remember TBR items and to suppress TBF items during encoding.

The second goal of Experiment 3 was to further examine age differences in source monitoring of TBR and TBF items as a function of emotion. Consistent with Gallant and Yang (2014), both Experiments 1 and 2 found that source recognition was better for positive TBR items than for positive TBF items in older adults. One possible explanation for this pattern of 
results is that older adults allocate more attention to processing positive items that are followed by a TBR cue, thus enhancing the binding of positive words with TBR cues. This would be consistent with their emotion-focused goals to prioritize remembering positive information, as outlined by theory surrounding the age-related positivity effect (Reed \& Carstensen, 2012). However, results from the first two experiments are limited in that conclusions cannot be drawn about differences in the allotment of attentional resources across emotion conditions during the encoding phase. Use of the ERP technique in Experiment 3 thus allows for further examining how attention is differentially allocated to TBR and TBF items as a function of emotion and whether this corresponded to subsequent source monitoring performance.

Furthermore, if older adults' memories for positive TBR items are indeed more richly represented due to better encoding of item and source, then their subjective recollection of these items may also be enhanced. To test this hypothesis in Experiment 3, the subjective strength of participants' recollection for both item-based recognition and source-based recognition was measured using an adaptation of the remember/know procedure (Tulving, 1985), one of the most widely used methods for probing the subjective experience of remembering. When we retrieve information from memory, it can be experienced either as a conscious recollection or simply as a feeling of familiarity that the event happened. The remember/know procedure probes these two types of experiences by asking participants to distinguish between items that they clearly remember relative to those that they only know occurred. A remember judgment is made when memory is vivid and accompanied with conscious recollection of contextual detail whereas a know judgment is made when participants only know that the item is old, but memory for context is weak (i.e., the item is familiar). Studies have shown that, in young adults, contextual features of information are better remembered for items that are reported as richly recollected relative to 
those that are reported as familiar (Kuhlmann \& Boywitt, 2015). Given that older adults show enhanced source memory for positive TBR items, it is possible that the strength of their subjective recollection may be greater for these items relative to the other conditions.

\section{Hypotheses}

Behavioural data. As in prior experiments, both age groups were expected to show directed forgetting for emotional and neutral words. With regard to source monitoring, results were expected to replicate Experiments 1 and 2, such that older adults' source memory would be elevated for positive TBR items relative to positive TBF items. As well, it was expected that older adults would tend to misattribute positive TBF and new items to TBR sources. Young adults' source monitoring was not expected to vary with valence.

Regarding subjective estimates of remembering for item-based recognition, consistent with Basden and Basden (1996, Experiment 3), recollection-based responses for TBR items should be elevated over TBF items due to distinctive processing of TBR items; however, responses based on familiarity should not be modulated by cue. This would suggest that participants retain greater contextual information for TBR than for TBF items. In older adults, recollection-based responses have been shown to be reduced, consistent with age-related declines in the ability to recollect episodic detail from memory (Anderson et al., 2008; Collette et al., 2014; Koen \& Yonelinas, 2016). As such, in the current experiment, these estimates may be reduced in older adults. However, if older adults' enhanced source recognition of positive TBR items is accompanied by greater recollection of contextual detail, then recollection-based responses may be selectively greater for these items relative to negative or neutral items. This would be manifest in an interaction of valence and cue for older adults' recollection-based responses to items with correct source identification. 
ERP data. The advantage of using ERP is the opportunity to examine processes evoked by both the word and cue even in the absence of a response. Analysis of word-based ERPs provides insight into whether emotion modulates processing resources prior to cue presentation and whether this influence continues following presentation of the cue. As such, ERP effects are organized according to those separately arising from the word and cue during encoding.

Word-based effects. The LPP component was of primary interest in examining the effect of emotion on word processing (Schupp et al., 2000). Consistent with Kisley and colleagues (2007), an age-related positivity effect was expected to emerge in ERPs elicited while studying words. This would be manifest in an interaction of age and valence on LPP amplitude, in which young adults show an elevated LPP in response to negative relative to positive or neutral items (e.g., Gallant \& Dyson, 2016). In contrast, older adults were expected to show a reduction in LPP amplitude to negative words. A positivity effect characterized by an enhanced LPP to positive over negative words in older adults was not predicted, given that the findings of Kisley and colleagues (2007) suggest a reduction in LPP amplitude for negative stimuli across the lifespan.

Cue-based ERP effects. Consistent with the literature (e.g., Paz-Caballero et al., 2004), young adults were expected to show enhanced parietal positivity in response to TBR cues and frontal positivity in response to TBF cues (Brandt et al., 2013; Gallant \& Dyson, 2016; Hauswald et al., 2010; Paz-Caballero et al., 2004). In contrast, given evidence for greater recruitment of frontal regions in fMRI (e.g., Davis et al., 2008; Rizio \& Dennis, 2014) and in ERP studies of aging (Friedman, 2003; O’Connell et al., 2012), older adults may show an anterior shift in parietal ERPs when attempting to encode TBR items relative to young adults. This would provide support for Rizio and Dennis' (2014) claim that divergent mechanisms underlie directed 
forgetting in young and older adults and may imply recruitment of cognitive control mechanisms to support encoding-related processes.

Consistent with prior research (Brandt et al., 2013; Gallant \& Dyson, 2016), it was expected that emotional influences on cue-related effects would be isolated to parietal activity following TBR words. As well, if older adults' source memory advantage for positive TBR items is driven by processes operating at encoding to enhance memory for items and their associated cues, it is possible that parietal ERPs may be elevated for this specific condition in older relative to young adults. Alternatively, if older adults' source advantage for positive TBR items is not driven by encoding-based processes, emotion may not influence cue-related amplitudes given that we did not find modulatory effects of emotion on directed forgetting in young or older adults in Gallant and Yang (2014) or in Experiments 1 and 2.

\section{Method}

\section{Participants}

The final sample included 28 young adults (ages 17 to 27) and 28 older adults (ages 65 to 84). All participants provided informed consent prior to participation and completed the battery of assessments described in the General Method (pages 40-42). As seen in Table 8, there were several group differences, in line with those reported in Gallant and Yang (2014) as well as in Experiments 1 and 2. Relative to young adults, older adults were more educated, had higher levels of positive affect, fewer symptoms of depression and anxiety, higher vocabulary, and slower processing speed (as indexed by fewer correct solutions on the DSST).

The data of six participants were excluded and replaced in line with the criteria outlined in the General Method (page 37). Four young adults and one older adult were excluded for scoring over the cutoff on the depression and/or anxiety subscales of the DASS-21; one older 
adult for scoring over the cut-off on the SBT; and one older adult who, after completing the memory task, indicated having severe issues seeing the stimuli on the screen due to early macular degeneration. The data of two additional participants (one young, one older) were replaced due to technical issues with the stimulus delivery program.

Table 8

Characteristics of the Final Sample in Experiment 3

\begin{tabular}{|c|c|c|c|c|}
\hline & $\begin{array}{l}\text { Young Adults } \\
\quad(n=28)\end{array}$ & $\begin{array}{l}\text { Older Adults } \\
\quad(n=28)\end{array}$ & & \\
\hline Characteristic & $M(S D)$ & $M(S D)$ & $p$-value & $d$ \\
\hline Age in Years & $20.35(2.66)$ & $72.39(5.05)$ & $<.001^{* *}$ & -12.89 \\
\hline Male / Female Ratio & $6 / 22$ & $11 / 17$ & .086 & - \\
\hline Years of Education & $13.91(1.76)$ & $17.13(2.99)$ & $<.001^{* *}$ & -1.31 \\
\hline Positive Affect ${ }^{a}$ & $27.14(8.66)$ & $35.75(6.92)$ & $<.001 * *$ & -1.10 \\
\hline Negative Affect ${ }^{a}$ & $13.68(3.98)$ & $12.21(3.37)$ & .143 & 0.40 \\
\hline Depression ${ }^{\mathrm{b}}$ & $7.14(4.70)$ & $4.71(4.36)$ & .050 & 0.54 \\
\hline Anxiety ${ }^{b}$ & $7.57(4.21)$ & $4.21(4.97)$ & $.016^{*}$ & 0.67 \\
\hline Shipley Vocabulary & $28.71(4.27)$ & $36.57(2.51)$ & $<.001 * *$ & -2.24 \\
\hline $\operatorname{DSST}^{\mathrm{c}}$ & $86.96(14.50)$ & $62.68(12.30)$ & $<.001 * *$ & 1.81 \\
\hline Short Blessed Test & - & $.79(1.66)$ & - & - \\
\hline
\end{tabular}

Note. Between-group comparisons were made using independent-samples $t$-tests apart from gender ratio, which was examined using Pearson's chi square. ${ }^{a}$ Measured using the Positive and Negative Affect Schedule; ${ }^{b}$ Measured with the DASS-21; ${ }^{\mathrm{c}}$ Scores reflect number of correct solutions. DSST $=$ Digit Symbol Substitution Test. ${ }^{*} p<.05 .{ }^{*} p<.001 . d=$ Cohen's d for standardized effect size estimate of the mean difference between groups.

\section{EEG Recording and Processing}

The EEG was recorded from $64 \mathrm{Ag} / \mathrm{AgCl}$ electrodes mounted in an elastic cap according to the International 10/20 configuration (see Figure 11). During recording, data were referenced to CMS (Common Mode Source) and DRL (Driven Right Leg). Horizontal electrooculogram 
(EOG) recordings were acquired via channels placed at the outer canthi and used to measure horizontal eye movements. The vertical EOG was recorded from channels placed at the inferior orbits and was used to detect eye blinks and vertical eye movements. The EEG and EOG activity were continuously digitized using a BioSemi ActiveTwo AD-Box (Bio-Semi; Wilmingston, NC) at a sampling rate of $512 \mathrm{~Hz}$.

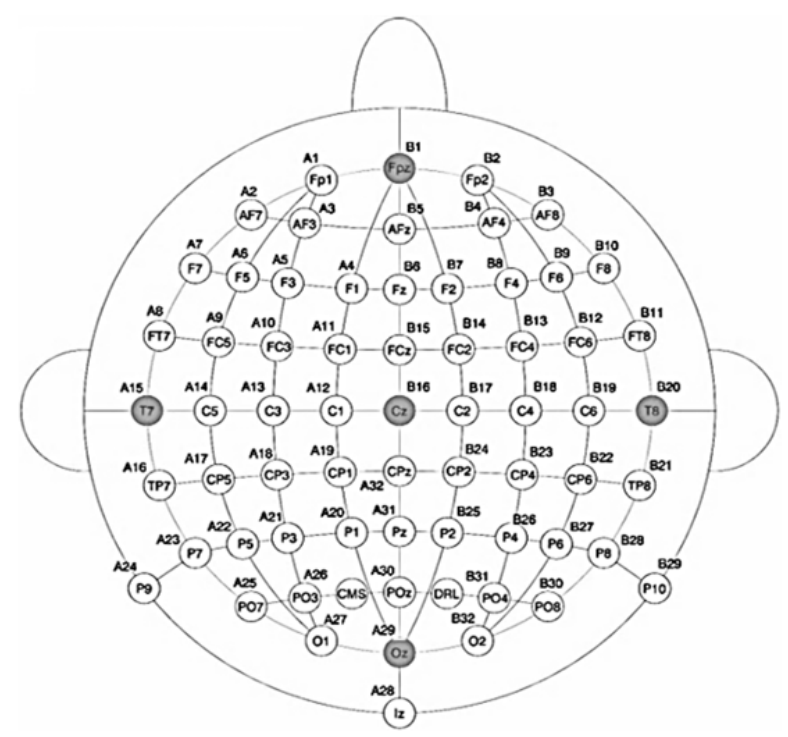

Figure 11. Layout of the 64-Channel International 10/20 system used to record EEG activity. Signal processing was performed in MATLAB using the EEGLAB/ERPLAB toolbox (Delorme \& Makeig, 2004; scripts for data processing can be found in Appendix XI). Data were downsampled to $256 \mathrm{~Hz}$ and bandpass filtered using a $0.1 \mathrm{~Hz}$ high-pass cutoff (12db/octave) to reduce low-frequency noise (e.g., drift) and a $30 \mathrm{~Hz}$ low-pass cutoff (24db/octave) to reduce high-frequency noise (e.g., muscle tension). The data were then re-referenced to the average of the left and right mastoids, $-200 \mathrm{~ms}$ baseline corrected, and segmented into $1000 \mathrm{~ms}$ epochs that were separately time-locked to the onset of the word and cue.

Artifacts were pruned from the segmented EEG data using independent components analysis (ICA). The goal of ICA is to decompose multivariate signals into additive but independent subcomponents (for a review, see Luck, 2014). During the ICA, the EEG and EOG 
data are submitted to a neural network in which a learning algorithm generates a matrix of independent components that are equal to the total number of EEG/EOG channels and correspond to the time course of the original data. These components may consist of true neural components or of artifacts such as eye blinks, eye movements, or muscle activity. Since the decomposed components are independent of each other, ICA is a good method for rejecting artifacts, as any rejected component would be independent of components indicative of neural activity. For the current EEG data, components indicative of horizontal (e.g., saccades) or vertical eye movements (e.g., blinks) were identified and rejected via visual inspection of the data. In doing so, the artifact is removed by setting its contribution to zero and recalculating the voltage at each electrode. Once the artifacts were pruned, the matrix of independent components was multiplied by the original matrix, recovering the original artifact-corrected EEG/EOG data. The resulting artifact-corrected data were then averaged across participants and mean amplitudes $(\mu \mathrm{V})$ were extracted for analysis.

\section{Materials}

Stimuli. The same list of 120 words as described in the General Method (page 38) was used in Experiment 3. To enhance the signal-to-noise ratio without increasing the number of stimuli, the 'old' list of 60 words was repeated during encoding, across two separate blocks of trials. Words were not repeated within blocks and the presentation order of items was rerandomized across blocks. ${ }^{9}$

Subjective experience of remembering. The remember/know procedure was adapted from Rajaram (1993) and modified to include 'Vivid' and 'Weak' judgments in place of

\footnotetext{
${ }^{9}$ Importantly, the ERP components of interest have been shown to be robust to multiple presentations of stimuli (Brown et al., 2012; Codispoti, Ferrari, \& Bradley, 2007). Pilot data analyses also revealed that repeated presentations of stimuli did not influence the behavioural directed forgetting effect (i.e., greater memory for TBR vs. TBF items).
} 
'Remember' and 'Know' judgments, respectively ${ }^{10}$. The critical task instructions were kept consistent and included the following: "Respond ' $\mathrm{V}$ ' for vivid if your recognition of the word is accompanied by a conscious recollection of its prior occurrence in the study list. A 'vivid' response comprises the ability to become consciously aware again of some aspect or aspects of what happened or what was experienced at the time the word was presented (e.g., what you were thinking and doing at the time). In other words, respond 'vivid' if the word brings back to mind a specific association, image, something more personal from the time of study, or something about its appearance. 'Weak' responses should be made when you recognize that the word was in the study list but you cannot consciously recollect anything about its actual occurrence, what happened or what was experienced at the time of its occurrence. In other words, respond 'weak' when you are certain of recognizing the words but these words fail to evoke any specific conscious recollection from the study list." Importantly, the instructions did not mention anything about recollecting the cue that was associated with the word; participants were only instructed to indicate 'vivid' if they remembered some sort of episodic detail about the word (e.g., a personal thought or association that they formed to help them remember). As such, participants could make a vivid judgment to a word even if they did not remember the specific cue that it was associated with.

\section{Procedure}

Encoding. Following the informed consent and EEG capping procedure, participants were seated in front of a desktop PC computer inside a soundproof booth. The trial procedure for the encoding task was identical to that of Experiment 2 (see Figure 7), with the exception that the

\footnotetext{
10 'Remember' and 'know' responses were initially used during pilot runs of the experiment. However, multiple participants indicated mixing up the ' $R$ ' source-based response and the ' $R$ ' recollection-based response. To prevent this confusion between responses and to facilitate performance on the recognition task, 'vivid' (V) and 'weak' (W) responses were used in place of 'remember' and 'know', respectively.
} 
high-value and low-value cues were respectively replaced with 'RRRR' and 'FFFF'. As well, the study list was presented twice, making for a total of 120 trials. Words were only presented once within each block and the presentation order of words differed across blocks. Following the first block of 60 words, there was a 20 -second break, after which the program proceeded to the next block of 60 words.

Filler task. Participants completed the DSST for two minutes.

Recognition. The recognition task consisted of 120 trials, including the 60 old words from the encoding task intermixed with 60 new words (words were not presented twice during recognition). Participants were instructed to identify whether each word had been associated with a cue to remember or a cue to forget during the study phase or if it was new. In addition, participants were asked to indicate the strength of their recollection for the words that they indicated as TBR or TBF. Specifically, if participants indicated a word as old (i.e., TBR or TBF), they were subsequently asked to judge whether their recollection of the word was Vivid or Weak according to the previously detailed instructions.

Each trial began with a fixation cross presented in the centre of the screen for $1000 \mathrm{~ms}$, replaced by a TBR, TBF, or new word that remained on the screen until a response was detected. Responses were made via the 'z', '’, or spacebar keys that were respectively labelled as "R", "F", and "New". If the participant responded 'new', the trial ended with a $500 \mathrm{~ms}$ ISI before proceeding to the next trial. If the word was identified as "R" or " $F$ ", a subsequent screen appeared with the probe "Vivid or Weak?" in the centre to which participants indicated the strength of their recollection for the word. Responses were made by pressing either the 'A' or ';' keys labelled as "V" and "W", respectively. Once a response was detected, a $500 \mathrm{~ms}$ ISI occurred before moving to the next trial. From start to finish, the EEG task took approximately 
25 minutes to complete. After the recognition task, the EEG cap was removed and the battery of assessments outlined in the General Method (pages 40-42) was administered.

\section{Data Analysis}

Behavioural data. For item recognition and source monitoring analyses, all relevant dependent variables (i.e., hits, false alarms, $A^{\prime}, B{ }^{\prime}$, CSIM scores, and source misattributions) were indexed according to procedures outlined in the General Method (pages 43-45). Resulting scores were submitted to a series of 3 (valence) $\times 2($ cue $) \times 2$ (age) ANOVAs. The cue factor was removed from analyses where dependent variables could not be distinguished by cue.

The current study also included 'vivid' and 'weak' judgments that were modeled after 'remember' and 'know' judgments, respectively (Rajaram, 1993; Tulving, 1985). These data were analyzed to infer the subjective strength of participants' recollection, with vivid judgments corresponding to recollection of contextual detail and weak judgments corresponding to familiar memories that lacked such detail. For item recognition, the proportions of vivid and weak judgments to TBR and TBF words (regardless of source response) were analyzed in separate 3 (valence) $\times 2$ (cue) $\times 2$ (age) ANOVAs. For source monitoring, only the items that were attributed to the correct source (i.e., TBR or TBF) were analyzed. As such, the proportions of vivid judgments were analyzed (because weak judgments were the inverse proportion).

ERP data. Analysis of word-based ERPs was modeled after existing literature on the LPP in response to emotion (Hauswald et al., 2010; Kisley et al., 2007; for a review, see Olofsson et al., 2008) as well as visual inspection of the data. This resulted in extraction of mean amplitudes from electrode sites P1, Pz, and P2 (see Figure 11) in the 600-900 ms epoch following onset of the word where LPP activity was maximal. Given that word-onset occurred prior to the cue, only the factors of valence and age were included in the ANOVA. 
The epochs and electrode sites selected for analysis of cue-related ERPs were also based on prior research (e.g., Brandt et al., 2013; Gallant \& Dyson, 2016; Hauswald et al., 2010; PazCabellero et al., 2003) as well as visual inspection of the data where maximal differences were observed. For frontal recordings, AF4, AFz, and AF3 electrode sites (see Figure 11) were selected for analysis. Following prior practice (Brandt et al., 2013; Hauswald et al., 2010; PazCaballero et al., 2004), analysis of frontal activity was segmented into three time windows including early (150-250 ms), middle (350-550 ms), and late (600-800 ms) epochs to better delineate the time course of processes involved in suppression of TBF items.

Parietal recordings were extracted from $\mathrm{P} 1, \mathrm{Pz}$, and $\mathrm{P} 2$ in the $350-550 \mathrm{~ms}$ time window to analyze ERPs associated with attempts to encode TBR items. This was again based on visual inspection of the data and prior literature (e.g., Brandt et al., 2013; Gallant \& Dyson, 2016; Hauswald et al., 2010; Paz Caballero \& Meñor, 2004). Mean cue-related amplitudes from frontal and parietal sites were analyzed in a series of ANOVAs as a function of valence, cue, and age.

Brain-behaviour correlations. If between- or within-subject differences were observed in ERP activity (e.g., age differences), correlation analyses were conducted to examine the association between ERP activity and behavioural performance on the item and source monitoring task.

\section{Results}

\section{Behavioural Data}

Item recognition performance. Table 9 displays mean recognition performance, including hits, false alarms, discrimination accuracy $\left(A^{\prime}\right)$, response bias $\left(B^{\prime}{ }_{D}\right)$, and proportions of vivid and weak judgments to TBR and TBF items. 
Table 9

Means and Standard Deviations for Item Recognition Indices in Experiment 3

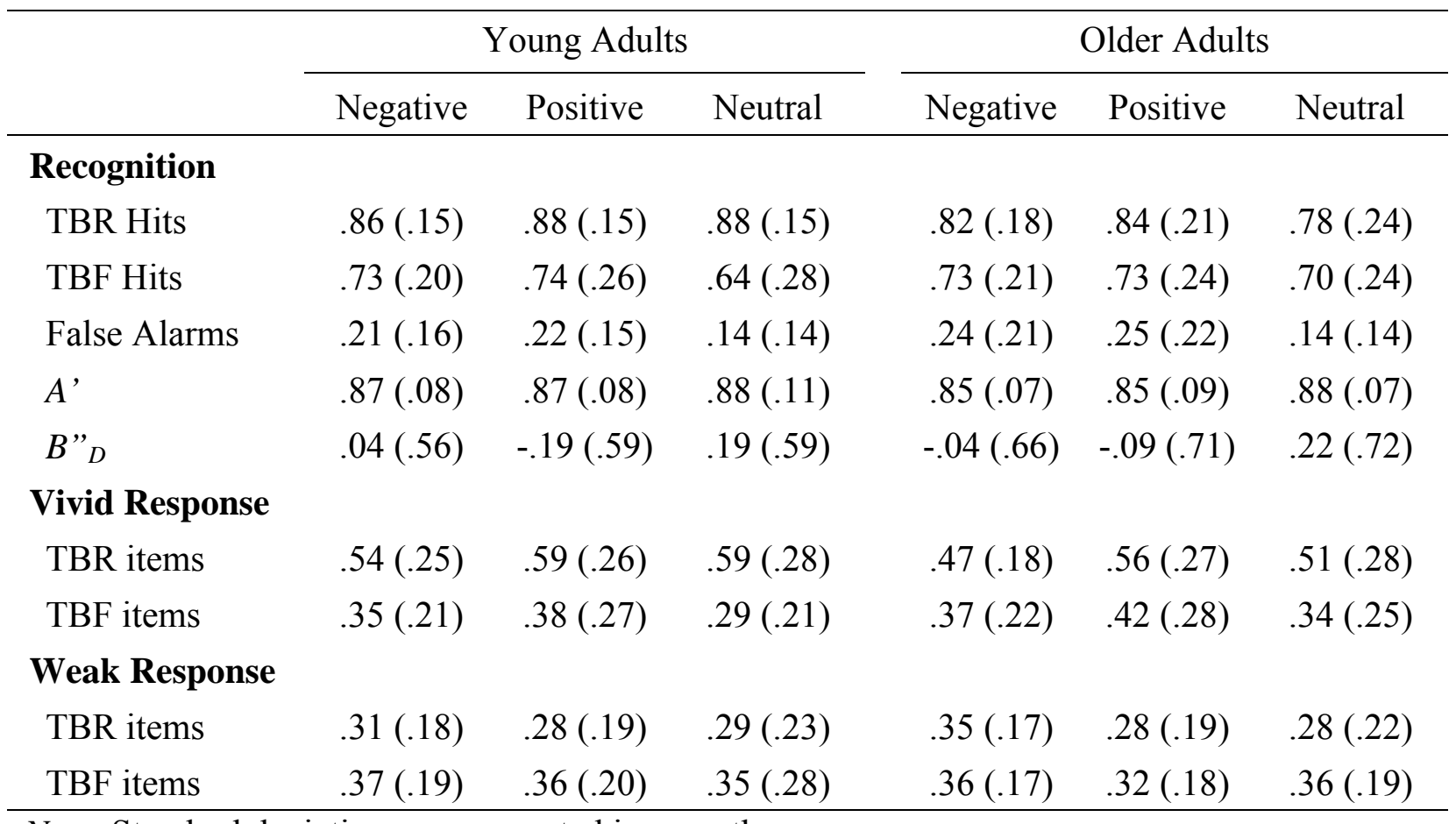

Note. Standard deviations are presented in parentheses.

The 3 (valence) $\times 2$ (cue) $\times 2$ (age) ANOVA on hit rates revealed a main effect of cue with greater hits for $\operatorname{TBR}(M=.84, S D=17)$ than for TBF items $(M=.71, S D=.20), F(1,54)=$ $52.97, p<.001, \eta_{p}^{2}=.50$. Hit rates also varied as a function of valence, $F(1.73,93.64)=3.25, p$ $<.05, \eta_{p}^{2}=.06$, driven by higher hits for positive $(M=.79, S D=.19)$ relative to neutral items $(M=.75, S D=.20 ; p<.05, d=0.35)$; hits for negative items $(M=.78, S D=.17)$ did not differ from either valence condition. An interaction of cue and age was also observed, $F(1,54)=5.20$, $p<.05, \eta_{p}^{2}=.08$. Planned comparisons revealed no between-group differences in recognition of TBR and TBF items ( $p s>.15)$; however, the magnitude of the within-group cue comparison was larger in young $\left(M_{\mathrm{TBR}}=.88, S D=.14\right.$ vs. $\left.M_{\mathrm{TBF}}=.70, S D=.22 ; p<.001, d=1.53\right)$ relative to older adults $\left(M_{\mathrm{TBR}}=.81, S D=.19\right.$ vs. $\left.M_{\mathrm{TBF}}=.72, S D=.20 ; p<.001, d=0.67\right)$ as inferred by age differences in Cohen's $d$ effect size estimates. 
A separate 3 (valence) $\times 2$ (age) ANOVA on false alarm rates revealed that neutral new items were less likely to be recognized as old $(M=.14, S D=.14)$ relative to negative $(M=.22$, $S D=.19 ; p<.01, d=0.76)$ and positive new items $(M=.24, S D=.19 ; p<.001, d=0.82)$ as revealed in a main effect of valence, $F(2,108)=25.26, p<.001, \eta_{p}^{2}=.32$. No other effects or interactions emerged in the analysis of hit and false alarm rates, $F s<2.15, p s>.12$.

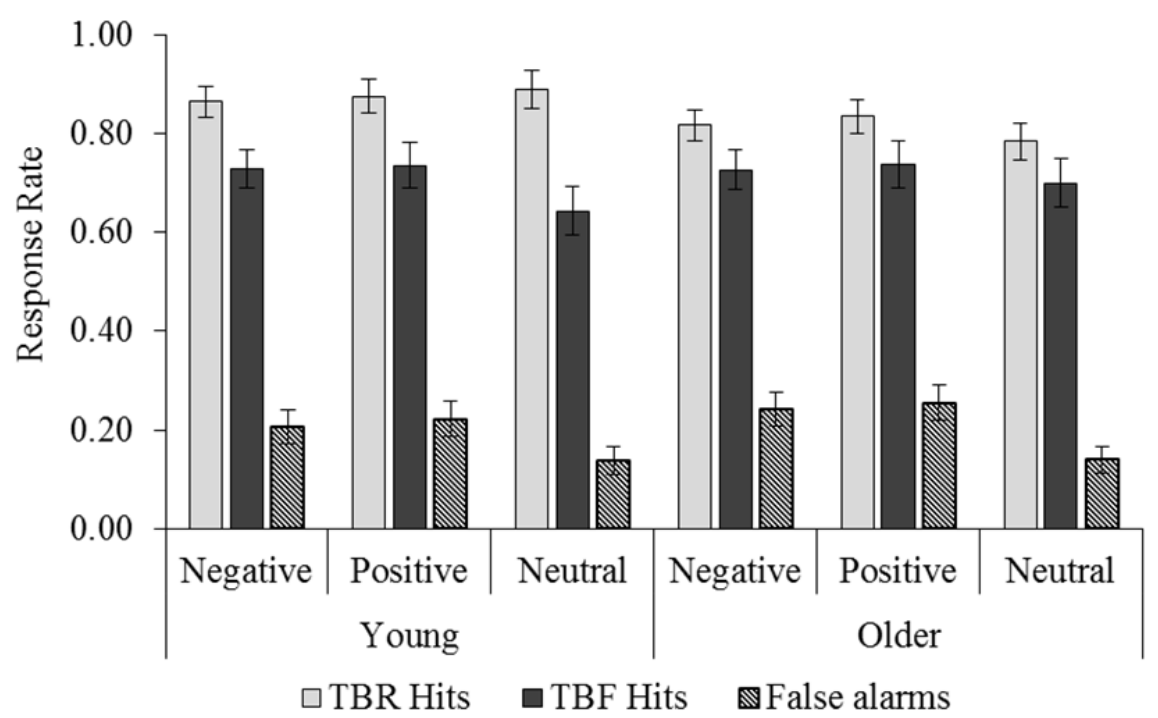

Figure 12. Proportions of hits and false alarms in Experiment 3 displayed as a function of age, valence, and cue. Error bars represent standard error of the mean.

According to a 3 (valence) $\times 2$ (age) ANOVA on discrimination accuracy, $A^{\prime}$ scores did not vary by valence nor age, Fs $<2.24$, ps $>.11$. A separate analysis of response bias scores revealed a main effect of valence, $F(1.78,92.42)=11.30, p<.001, \eta_{p}^{2}=.18$, such that $B{ }_{D}$ scores were higher for neutral items $(M=.21, S D=.64)$ than for positive $(M=-.16, S D=.65 ; p$ $<.001, d=0.65)$ or negative items $(M=-.01, S D=.61 ; p<.01, d=0.37)$. One-sample $t$-tests confirmed a conservative response bias for neutral items, as $B{ }_{D}{ }_{D}$ scores differed from zero in the positive direction, $t(55)=2.42, p<.05$. Response bias for positive items was marginally different from zero in the negative direction, $t(55)=-1.87, p=.07$, whereas $B{ }_{D}{ }_{D}$ for negative items did not differ from zero $(p=.89)$. No other effects were observed, Fs $<.88, p s>.45$. 
Taken together, the item recognition analyses confirmed a directed forgetting effect with higher hit rates for TBR than TBF items, the magnitude of which was larger for young than for older adults. Overall, relative to neutral items, emotion increased both hits (particularly positive items) and false alarms. Conservative response biases indicated that participants required more memory evidence before responding 'old' to neutral items, relative to positive or negative items. In contrast, whereas no response bias was observed for negative items, analyses suggest a potential liberal bias to respond 'old' to positive items; however, this latter finding should be interpreted with caution as it did not reach the threshold for statistical significance.

Vivid and weak judgments for item recognition. The ANOVA on vivid judgments indicated that TBR items $(M=.55, S D=.23)$ received more vivid judgments than TBF items ( $M$ $=.36, S D=.20), F(1,53)=49.02, p<.001, \eta_{p}^{2}=.48$. Positive items also received more vivid judgments $(M=.48, S D=.22)$ than negative $(M=.43, S D=.18 ; p<.01, d=0.35)$ or neutral items $(M=.43, S D=.22 ; p<.01, d=0.35), F(2,106)=5.06, p<.01, \eta_{p}^{2}=.09$. A two-way interaction of age and cue implied that vivid judgments to TBR and TBF items were differentially influenced by age, $F(1,53)=3.57, p=.06, \eta_{p}^{2}=.06$. Planned comparisons revealed no between-group differences in vivid judgments for TBR and TBF items ( $p s>.27$ ); however, based on effect size (Cohen's $d$ ), the magnitude of the cue effect on vivid judgments was larger for young $\left(M_{T B R}=.58, S D=.24\right.$ vs. $\left.M_{T B F}=.34, S D=.19 ; p<.001, d=1.22\right)$ than for older adults $\left(M_{T B R}=.45, S D=.22\right.$ vs. $\left.M_{T B F}=.31, S D=.18 ; p<.01, d=0.73\right)$. Cue also influenced weak judgments, with higher responses for TBF $(M=.35, S D=.15)$ relative to TBR items $(M=.29, S D=.16), F(1,53)=4.88, p<.05, \eta_{p}^{2}=.08$. No other effects emerged, $F s<$ $2.37, p s>.10$. 
Given that a directed forgetting effect was observed only for vivid judgments, this may imply a larger contribution of recollection-based processes to the directed forgetting effect than of familiarity-based processes. In contrast, participants' recognition of TBF items may be based on memories that are familiar as opposed to high in recollection.

Source monitoring performance. Table 10 displays source monitoring performance including average CSIM scores, misattributions to new items, and proportions of vivid and weak judgments for correct source recognition across age groups.

Table 10

Means and Standard Deviations for Source Monitoring Indices in Experiment 3

\begin{tabular}{|c|c|c|c|c|c|c|}
\hline & \multicolumn{3}{|c|}{ Young Adults } & \multicolumn{3}{|c|}{ Older Adults } \\
\hline & Negative & Positive & Neutral & Negative & Positive & Neutral \\
\hline \multicolumn{7}{|c|}{ Correct Attributions ${ }^{\text {a }}$} \\
\hline TBR items as TBR & $.69(.17)$ & $.73(.16)$ & $.78(.16)$ & $.61(.22)$ & $.80(.16)$ & $.74(.20)$ \\
\hline TBF items as TBF & $.70(.23)$ & $.67(.25)$ & $.75(.21)$ & $.67(.26)$ & $.59(.27)$ & $.66(.31)$ \\
\hline \multicolumn{7}{|c|}{ Source False Alarms ${ }^{\text {b }}$} \\
\hline New items as TBR & $.06(.08)$ & $.08(.09)$ & $.03(.05)$ & $.08(.12)$ & $.11(.13)$ & $.05(.06)$ \\
\hline New items as TBF & $.15(.12)$ & $.14(.13)$ & $.10(.11)$ & $.16(.18)$ & $.14(.15)$ & $.09(.11)$ \\
\hline \multicolumn{7}{|l|}{ Vivid Responses } \\
\hline TBR items & $.72(.32)$ & $.76(.24)$ & $.69(.32)$ & $.70(.30)$ & $.74(.23)$ & $.71(.31)$ \\
\hline TBF items & $.46(.29)$ & $.42(.31)$ & $.51(.33)$ & $.36(.32)$ & $.36(.34)$ & $.36(.37)$ \\
\hline \multicolumn{7}{|l|}{ Weak Responses } \\
\hline TBR items & $.28(.32)$ & $.24(.24)$ & $.31(.32)$ & $.30(.30)$ & $.26(.23)$ & $.29(.31)$ \\
\hline TBF items & $.54(.29)$ & $.58(.31)$ & $.49(.33)$ & $.64(.32)$ & $.64(.34)$ & $.64(.37)$ \\
\hline
\end{tabular}

Note. Standard deviations are presented in parentheses; ${ }^{a}$ represents CSIM score according to the calculation on Page 45; ${ }^{b}$ represents proportion of misattributions of sources to new items.

The analysis of CSIM scores revealed a main effect of valence, $F(2,108)=3.96, p<.05$, $\eta_{p}^{2}=.06$, driven by reduced source recognition of positive $(M=.67, S D=.15)$ relative to neutral items $(M=.73, S D=.16 ; p<.01, d=0.35)$; the other conditions did not differ $(p s>.13)$. The 
interaction of valence and cue was significant, $F(2,108)=6.37, p<.01, \eta_{p}^{2}=.11$, and qualified by a marginal three-way interaction between age, valence, and cue, $F(2,108)=2.47, p=.08, \eta_{p}^{2}$ $=.04$ (see Figure 13). Planned comparisons showed no between-group differences in correct source recognition $(p s>.12)$. However, valence differentially influenced source monitoring across cues for older, $F(2,54)=7.53, p<.01, \eta_{p}^{2}=.22$, but not young adults $(p=.60)$. Within the older group, source monitoring was greater for positive than negative TBR items $(p<.001, d$ $=0.71)$, the latter of which was reduced relative to neutral TBR items $(p<.01, d=0.57)$. In contrast, older adults' source recognition of TBF items did not differ across emotions ( $p s>.13$ ), nor did it differ from young adults ( $p s>.25$ ). No other effects emerged, $F s<2.47, p s>.14$.

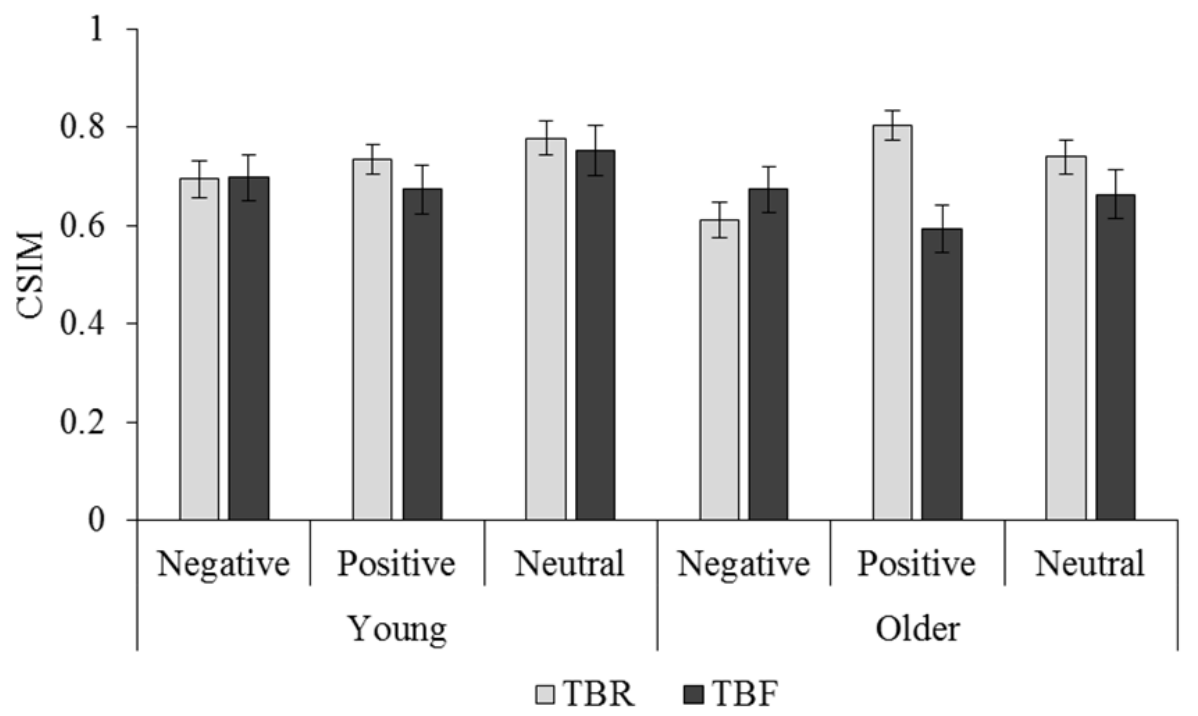

Figure 13. Proportions of CSIM scores in Experiment 3 as a function of age, valence, and cue. Error bars represent standard error of the mean.

Next, source misattributions were analyzed as a function of valence, cue, and age. Source misattributions were reduced for neutral $(M=.07, S D=.07)$ relative to negative $(M=.11, S D=$ $.09 ; p<.001, d=0.78)$ and positive items $(M=.12, S D=.09 ; p<.001, d=1.05), F(2,108)=$ 26.26, $p<.001, \eta_{p}^{2}=.32$. Overall, misattributions were higher for $\operatorname{TBF}(M=.13, S D=.12)$ 
relative to TBR words $(M=.07, S D=.08), F(1,54)=14.81, p<.001, \eta_{p}^{2}=.22$. Different from prior experiments, valence and cue did not differentially influence misattributions across groups (ps $>.21$ ). No other effects or interactions were observed, Fs $<1.58, p s>.21$.

In sum, these analyses suggest that for TBR items, older adults had better source memory for positive TBR relative to negative or neutral TBR words. No age differences were observed in correct source recognition or misattributions as a function of valence.

Vivid and weak judgments for correct source recognition. The 3 (valence) $\times 2$ (cue) $\times 2$ (age) ANOVA on vivid judgments to TBR and TBF items that were attributed the correct source varied by cue, as illustrated by greater vivid judgments toward TBR $(M=.72, S D=.24)$ relative to $\mathrm{TBF}$ items $(M=.41, S D=.26), F(1,53)=44.96, p<.001, \eta_{p}^{2}=.46$, suggesting that recollection of contextual detail contributed more to participants' correct source identification of TBR relative to TBF items. No other effects were observed, $F s<1.35$, ps $>.26$.

\section{ERP Data: Word Effects}

The 3 (valence) $\times 2$ (cue) $\times 2$ (age) ANOVA on word-related LPP amplitude revealed a two-way interaction between valence and age, $F(1.34,70.81)=5.99, p<.01, \eta_{p}^{2}=.10$. Planned contrasts revealed that young adults' LPP amplitude was enhanced for negative $(M=3.78 \mu \mathrm{V}$, $S D=4.99)$ relative to positive $(M=.86 \mu \mathrm{V}, S D=3.75 ; p<.05, d=0.42)$ as well as neutral words $(M=.94 \mu \mathrm{V}, S D=4.68 ; p=.08, d=0.35)$, although this latter difference was marginally significant and so should be interpreted with caution (see Figure 14). In contrast, older adults showed a marginally reduced LPP in response to negative $(M=2.71 \mu \mathrm{V}, S D=2.95)$ relative to neutral words $(M=4.54 \mu \mathrm{V}, S D=7.01 ; p=.06, d=0.36)$. Between groups, older adults showed higher LPP responses to both positive $(p<.05, d=0.63)$ and neutral items $(p<.05, d=0.62)$; no group difference was observed between negative items $(p=.52)$. No other effects emerged $F<$ 
2.98 , ps $>.10$. No correlations emerged between LPP amplitude and subsequent item- or sourcebased recognition across valence conditions in either group $(r s>-.22$ and $<.04, p s>.11)$.

Young Adults

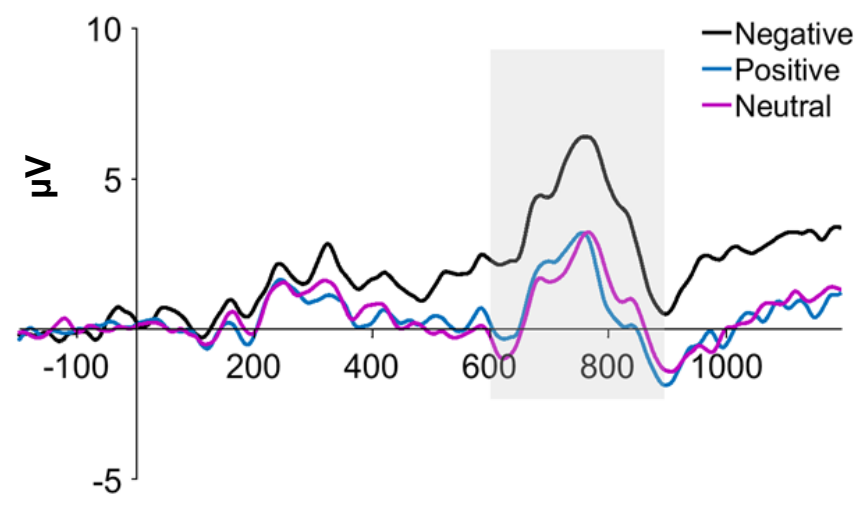

Older Adults

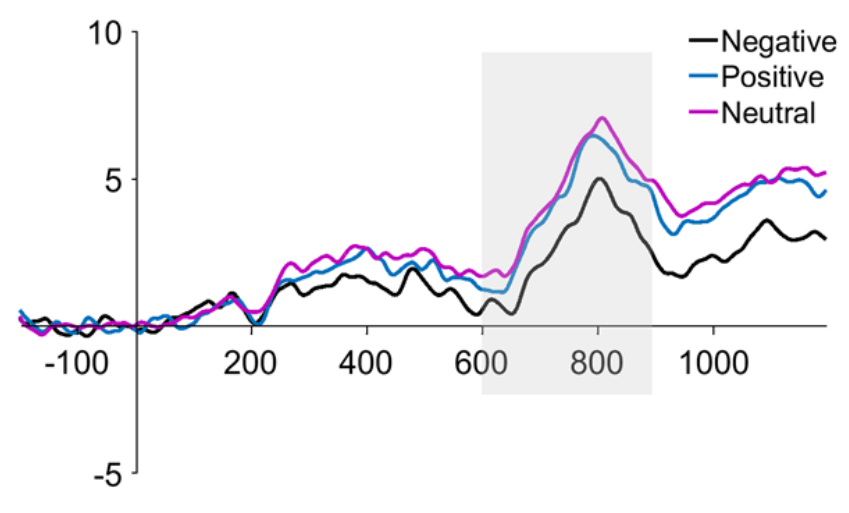

Figure 14. Grand averaged ERPs from P1, Pz, and P2 electrodes, time-locked to word onset. Time is on the x-axis and amplitude differences are highlighted in the 600-900 ms epoch.

\section{ERP Data: Cue Effects}

Parietal activity. The 3 (valence) $\times 2$ (cue) $\times 2$ (age) ANOVA revealed more positive cue-related ERPs to TBR $(M=7.39 \mu \mathrm{V}, S D=4.58)$ than to TBF items $(M=6.66 \mu \mathrm{V}, S D=$ 4.71), $F(1,54)=5.35, p<.05, \eta_{p}^{2}=.10$ (see Figure 15). No other effects or interactions were observed, $F s<1.17, p s>$.31. Parietal ERPs elicited by TBR cues were also positively correlated with both item recognition of TBR items $(r=.49, p<.001)$ and source recognition of TBR items $(r=.37, p<.01)$. As well, ERPs evoked by TBF cues were positively correlated with recognition of TBF items $(r=.30, p<.05)$. These correlations imply that as the amplitude of parietal positivity for TBR and TBF cues increased, so too did overall item and source recognition of these items. No other correlations with behaviour were observed, $r s>.18$ and $<.23$, ps $>.11$. Altogether, these analyses suggest that young and older adults recruited the same pattern of parietal activity in response to cues that is typically observed in the literature (e.g., Paz-Caballero et al., 2004) and that this activity may be important for subsequent recognition performance. 

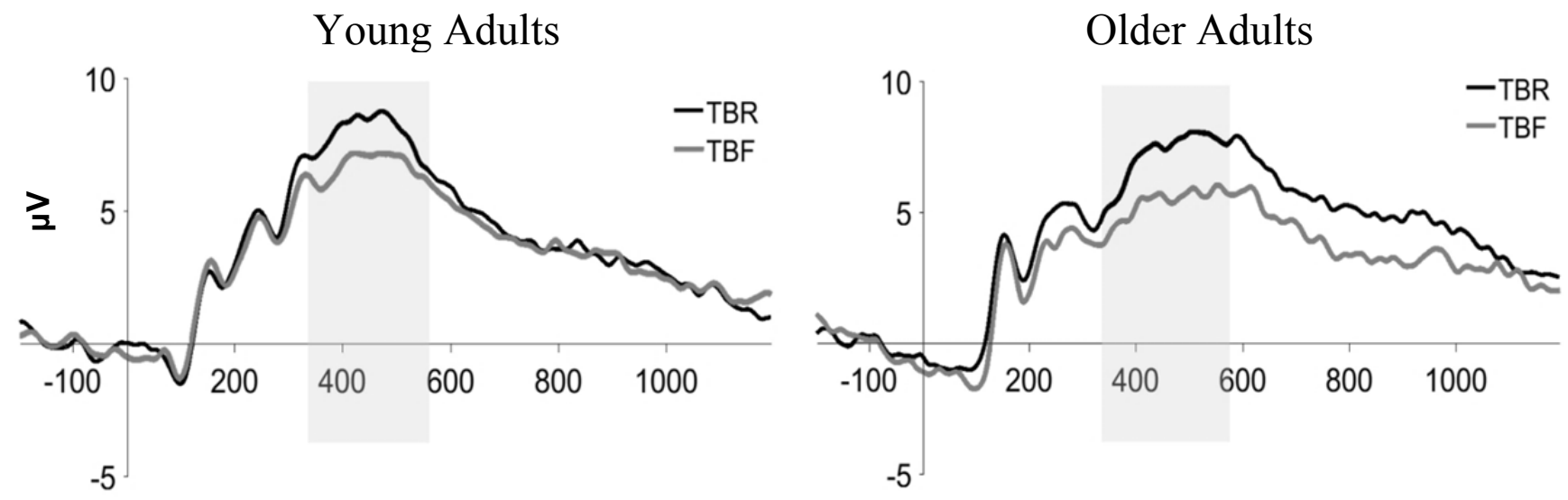

Figure 15. Grand averaged ERPs extracted from P1, Pz, and P2 electrode sites, time-locked to cue onset, and collapsed across valence. Time is on the x-axis and cue-related amplitude differences are highlighted in the 350-550 ms epoch.

Frontal activity ${ }^{11}$. Based on prior literature (e.g., Hauswald et al., 2010; Pires et al., 2014), results for frontal activity were separated into three segments including early (150-250 $\mathrm{ms})$, middle (350-550 ms), and late (600-800 ms) latencies following onset of the memory cue.

Early: 150-250 ms. In the early epoch, mean amplitudes were higher for TBF $(M=3.04$ $\mu \mathrm{V}, S D=3.27)$ relative to $\mathrm{TBR}$ cues $(M=2.23 \mu \mathrm{V}, S D=3.73), F(1,53)=4.54, p<.05, \eta_{p}^{2}=$ .08 . There was also a marginal interaction of age and cue, $F(1,53)=3.68, p=.06, \eta_{p}^{2}=.07$. Planned comparisons revealed that young adults had more positive amplitudes for TBF ( $M=$ $2.81 \mu \mathrm{V}, S D=3.27)$ relative to TBR items $(M=1.45 \mu \mathrm{V}, S D=3.81 ; p<.05, d=0.44)$ whereas older adults $\left(M_{\mathrm{TBF}}=3.29 \mu \mathrm{V}, S D=3.32\right.$ vs. $\left.M_{\mathrm{TBR}}=3.12 \mu \mathrm{V}, S D=3.49\right)$ did not show this difference ( $p=.66$; Figure 16). No between-group differences emerged for cue-related amplitudes $(p>.10)$ and no other effects were observed in the analysis, $F s<1.31, p s>.26$. No correlations were observed between frontal ERPs in this time window and behavioural performance for either age group, $r s>-.24$ and $<.19$, ps $>.21$.

\footnotetext{
${ }^{11}$ One older adult's data was removed from these analyses as their mean amplitudes were more than 2.5 standard deviations over the group mean. As such, the degrees of freedom are slightly reduced in this set of analyses.
} 
Middle: $350-550 \mathrm{~ms}$. In the middle epoch, older adults' frontal ERPs $(M=6.17 \mu \mathrm{V}, S D=$ 4.66) were more positive than those of young adults $(M=2.34 \mu \mathrm{V}, S D=4.04), F(1,53)=10.26$, $p<.01, \eta_{p}^{2}=.17$. ERPs elicited by cues, however, differed across age groups, as illustrated by an interaction of these factors, $F(1,53)=4.50, p<.05, \eta_{p}^{2}=.08$. Planned comparisons revealed more positive amplitudes for $\mathrm{TBF}(M=3.04 \mu \mathrm{V}, S D=4.38)$ relative to TBR cues $(M=1.63 \mu \mathrm{V}$, $S D=4.10 ; p<.01, d=0.55)$ in young adults whereas older adults showed no difference in cuerelated activity $\left(M_{\mathrm{TBF}}=6.08 \mu \mathrm{V}, S D=4.89\right.$ vs. $\left.M_{\mathrm{TBR}}=6.27 \mu \mathrm{V}, S D=4.87 ; p=.74\right)$. Relative to young adults, older participants showed more positive amplitude to both $\operatorname{TBR}(p<.001, d=$ 1.03) and TBF cues $(p<.05, d=0.65)$; however, the magnitude of this age difference was larger for TBR cues as inferred by Cohen's $d$ effect size. No other effects emerged, Fs $<2.58, p s>.11$.

Whereas no correlations were observed for young adults $(r s>-.18$ and $<.24, p s>.22)$, older adults' frontal ERPs elicited by TBR cues were positively correlated with recognition of TBR items $(r=.43, p<.03)$. There were no other correlations $(r s>.11$ and $<.25, p s>.12)$.

Late: $600-800 \mathrm{~ms}$. In the final epoch, older adults' overall mean amplitudes $(M=6.54$ $\mu \mathrm{V}, S D=6.40)$ were more positive than young adults' $(M=1.43 \mu \mathrm{V}, S D=3.61), F(1,53)=$ $12.33, p<.01, \eta_{p}^{2}=.19$. The impact of cue was sustained into this later epoch, with more positive ERPs for TBF $(M=4.82 \mu \mathrm{V}, S D=6.58)$ than TBR cues $(M=3.04 \mu \mathrm{V}, S D=5.85), F(1$, $53)=5.91, p<.05, \eta_{p}^{2}=.10$. Unlike the middle epoch, cue-related amplitudes were not differentially influenced by age $(p=.24)$. No other effects were observed, $F s<1.53$, $p s>.22$.

Young adults' ERPs were not correlated with subsequent recognition performance ( $r s>$ .22 and $<.23$, ps $>.16$ ). In contrast, older adults' frontal ERPs elicited by TBR cues were positively correlated with subsequent recognition of TBR items $(r=.40, p<.05)$. No other brain-behaviour correlations were observed $(r s>-.03$ and $<.26$, ps $>.08)$. 


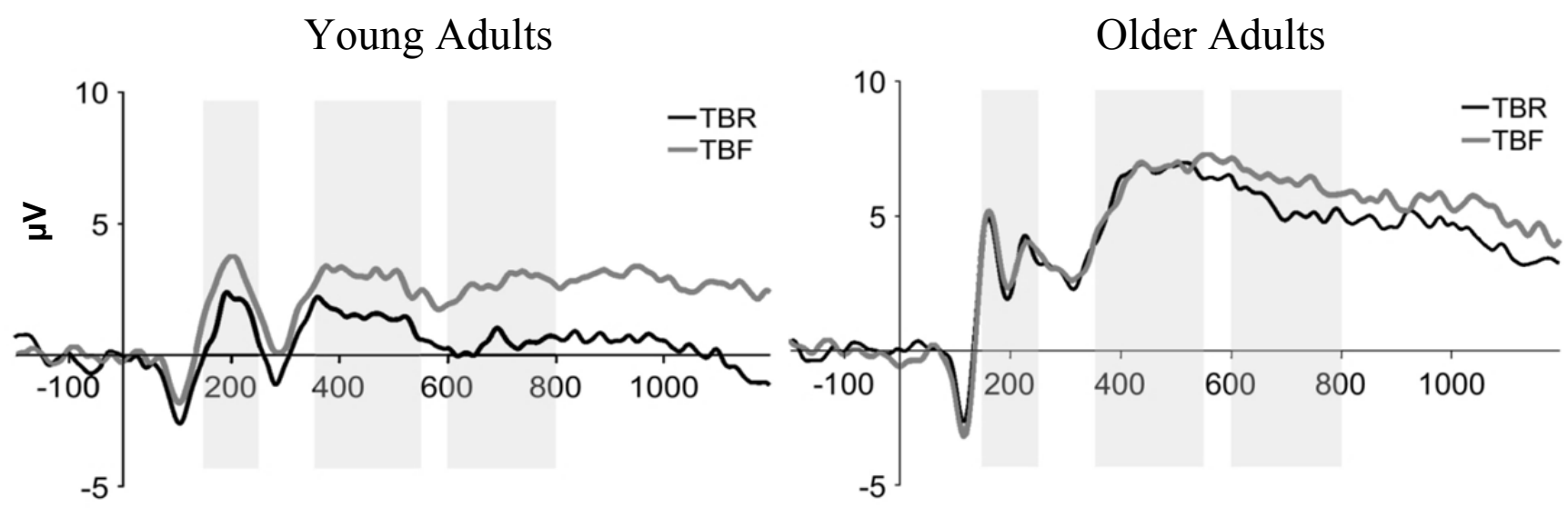

Figure 16. Grand averaged ERPs from AF3, AFz, and AF4 electrodes, time-locked to cue onset, and collapsed across valence conditions. Time is on the x-axis and amplitude differences are highlighted in the $150-250 \mathrm{~ms}, 350-550 \mathrm{~ms}$, and $600-800 \mathrm{~ms}$ epochs.

To summarize, the cue-related analyses of frontal activity imply age differences in the recruitment of frontal activity during directed forgetting of TBR and TBF words. Whereas young adults showed the expected increase in frontal positivity for TBF relative to TBR items across time windows, older adults showed no such differentiation in their cue-related activity at both early and middle latencies. Not until the later time window did older adults show the expected enhanced frontal activity for TBF relative to TBR items. In the middle time window, there was also evidence for an increase in older relative to young adults' frontal activity in response to TBR cues. Importantly, this activity correlated with older adults' recognition of both TBR and TBF items, suggesting that as these frontal amplitudes increased, so did recognition.

\section{Analysis of Individual Differences}

Several differences were observed between groups on the cognitive and affective measures as illustrated in Table 8. As such, correlations were calculated within-groups to determine whether these sample differences related to performance on the dependent variables that also showed age differences. This analysis revealed a positive correlation between older adults' performance on the DSST and their recognition of TBR items $(r=.43, p<.05)$, 
suggesting that higher processing speed may correspond to better recognition of TBR words. No other correlations were observed for young adults $(r s>-.28$ and $<.31, p s>.12)$. A mediation analysis was performed, with DSST scores entered as a mediator, to determine whether processing speed might mediate the effect of age on recognition of TBR items (Hayes, 2012). The analysis revealed that scores on the DSST were a significant predictor of TBR recognition ( $p$ $<.01)$. As well, the effect of age on recognition of TBR items was no longer significant ( $p=$ .35). This may imply that general age-related slowing in psychomotor speed, as indexed by DSST scores, may have played a role in older adults' recognition of TBR items.

\section{Discussion}

The goal of Experiment 3 was to examine age differences in brain activity underlying young and older adults' directed forgetting and source monitoring of emotional and neutral information. As in Experiments 1 and 2, a directed forgetting task was used to measure cognitive control over TBR and TBF words that varied in emotional tone. To index brain activity, the ERP technique was used, given its utility for tracking event-related brain processes as they unfold over time. Moreover, the subjective strength of participants' recollection for item-based and source-based recognition was measured by asking participants to determine whether their recognition of items was accompanied by a vivid recollection of contextual detail or (2) a nonspecific feeling of familiarity in which memory for context is weak. The main findings are discussed in the following sections, organized according to behavioural and ERP results.

\section{Behavioural Findings}

Directed forgetting results mimicked those reported in Gallant and Yang (2014), with enhanced recognition of TBR relative to TBF words on the directed forgetting task across age groups. Although directed forgetting was intact in older adults, the magnitude of the effect was 
reduced relative to young adults supporting evidence in favour of age-related declines in cognitive control (e.g., Andrés et al., 2004; Collette et al., 2009; Gallant \& Yang, 2014; Hogge et al., 2008; Titz \& Verhaeghen, 2010; Zacks et al., 1996). Of primary interest, however, is the finding that both young and older adults could control encoding of TBR and TBF items that varied in emotion, which replicates Experiments 1 and 2 as well as prior literature (e.g., Berger et al., 2016; Gallant \& Yang, 2014).

With regard to subjective recollection of item-based recognition, more of the vivid judgments were attributed to TBR than to TBF items whereas no effect was observed for weak judgments. This finding is consistent with prior directed forgetting research using the remember/know paradigm (e.g., Basden \& Basden, 1996, Experiment 3; Collette et al., 2014; Gardiner et al., 1994; Rizio \& Dennis, 2014), and implies a greater contribution of recollectionbased processes to the retrieval of TBR words than to TBF words, and thus to the directed forgetting effect. When compared to young adults, the magnitude of the cue effect on vivid judgments was reduced in older adults, suggesting that recollection may have contributed less to older adults' directed forgetting than it did to their young counterparts. These results fall in line with research documenting an age-related reduction in the ability to consciously recollect memories rich in contextual detail (Anderson et al., 2008; Koen \& Yonelinas, 2016). In contrast, TBF items were more likely to be associated with subjective feelings of familiarity that lacked memory for contextual features, which implies a greater contribution of familiarity-based processes (i.e., recognition lacking conscious recollection) to the recognition of TBF items. When examining source monitoring performance, an interesting pattern of results emerged relative to the preceding experiments as well as Gallant and Yang (2014). In the current study, older adults showed enhanced correct source recognition for positive TBR items than for 
positive TBF items; however, in contrast to Experiments 1 and 2, older adults' source recognition of positive TBF items was not reduced relative to the other valence conditions. Related to this is the finding that the source misattribution bias to attribute positive TBF and new items to TBR sources did not come to fruition in this study. Rather, older adults showed the expected tendency to misattribute new items to TBF sources, regardless of valence. These findings imply that older adults were better able to use their knowledge of cognitive operations to inform their source monitoring performance in the current experiment. One possibility is that this divergence in results was driven by the repetition of items during the encoding phase. Although items were repeated to enhance the signal-to-noise ratio in the EEG data, it may have strengthened knowledge of cognitive operations associated with each word as participants were provided with an additional opportunity to engage selective rehearsal mechanisms for each TBR item and inhibitory processes for each TBF item. As a result, by increasing records of cognitive operations for each item, older adults may have been better able to rely on this metacognitive knowledge during source monitoring, resulting in fewer misattributions of positive TBF items to TBR sources and thus increased source recognition for positive TBF items.

Consistent with Experiments 1 and 2, older adults also had higher source memory for positive TBR words relative to the other conditions. Interestingly, despite enhanced source recognition for positive TBR items, older adults did not show a corresponding increase in their conscious recollection of these items. These results suggest that although older adults could correctly associate positive items with TBR cues, their memory for these items was not any richer in contextual detail than was the case in the other valence conditions. While source memory is often considered a form of objective recollection, it has been shown to diverge from subjective estimates of recollection in terms of both behaviour and neural responses (Spaniol et 
al., 2009). This may be due to criterial differences that are involved in making each form of judgment. Source recognition involves a more specific form of recollection in which the participant must search for detail that the experimenter has deemed central to the event or item being retrieved (in this case, the associated memory cue). The source monitoring framework (Johnson et al., 1993) has also suggested that factors other than the memory trace may contribute to a participant's source decision, such as their beliefs or general knowledge about the task (e.g., knowledge that TBR cues $=$ strong memory, TBF cues $=$ weak memory). In contrast, subjective recollection judgments may involve recollection of more peripheral details, such as thoughts or feelings experienced during encoding that are not central to the specific item. Taken together, the current results may therefore suggest that positive information enhances older adults' ability to recollect central contextual details of items (i.e., the associated TBR cue), but that this does not necessarily correspond to more vivid memories that are rich in contextual details that are peripheral to the item (i.e., thoughts or feelings).

\section{ERP Findings}

During word presentation, young and older adults' LPP activity varied according to the emotional tone of words. Whereas young adults showed an enhanced LPP to negative over positive words, the opposite was observed for older adults, who showed a reduction in their LPP response to negative items. Given the proposed functional significance of the LPP (Olofsson et al., 2008; Schupp et al., 2000), these results may thus suggest an increase in attention allocation toward negative stimuli in young adults that is reduced in older adults. This pattern of results falls in line with the age-related positivity effect (Mather \& Carstensen, 2005) and is consistent with evidence from previous investigations showing a reduction in LPP responses to negative stimuli across the lifespan (Kisley et al., 2007). These findings may thus reflect a motivation to 
shift attention away from negative words in older adults, as prior research has indicated that the LPP likely reflects top-down goal-directed attention processes (Brown et al., 2012; Liu, Huang, McGinnis, Keil, \& Ding, 2012). From a methodological standpoint, the word-related LPP results also demonstrate that emotional words were indeed processed differently from neutral words in the context of these experiments. This is important as it implies that the null impact of emotion on directed forgetting cannot be attributed to words that failed to elicit an emotional response.

When not considering emotion, cue-related activity demonstrated distinct ERPs underlying TBR and TBF cues in both young and older adults. Regarding parietal activity, TBR cues evoked more positive amplitudes than TBF words, regardless of age or emotion. This cuerelated parietal activity was also positively correlated with subsequent item and source recognition of these items, highlighting the functional significance of this neural response. These results are in line with prior studies (e.g., Gallant \& Dyson, 2016; Hauswald et al., 2010; PazCaballero et al., 2004), which have similarly shown increased positivity within this time window following cues to remember. The parietal ERPs in this time window are typically associated with strategic allocation of attention that facilitates memory performance (Polich, 2007), and so may reflect selective rehearsal mechanisms that work to prioritize TBR items in the current experiment.

In contrast, age differences emerged when examining frontal-based ERPs. Beginning in early time windows, frontal activity was more positive following a TBF relative to TBR cue in young adults, which has been argued to reflect the onset of a blocking mechanisms to suppress TBF items (Paz-Caballero et al., 2004). This frontal cue effect was consistent for young adults throughout all time windows, consistent with Gallant and Dyson (2016) who identified frontal positivity in response to TBF cues that was sustained until $850 \mathrm{~ms}$ after cue onset. Older adults, 
however, diverged from this pattern of results. In the early and middle time windows, the expected cue differentiation in frontal activity (i.e., TBF $>$ TBR) was not apparent in the older group. Instead, when compared to young adults, the older group showed increased frontal positivity following TBR cues in the middle time window (350-550 ms, consistent with the P3b latency), that was positively associated with their subsequent recognition of these items. This correlation, which was not observed in young adults, thus implies that older adults' increased frontal activity may have served a functional purpose by facilitating memory for target stimuli in an age-specific manner. This pattern of findings fits the criteria for 'successful compensation' (Cabeza \& Dennis, 2012; Grady, 2008, 2012), suggesting that older adults may recruit additional resources in frontal regions to compensate for declines in other parts of the brain.

The age differences in frontal ERPs described above are also quite consistent with Rizio and Dennis' (2014) fMRI investigation, in which older adults showed greater activity in prefrontal and frontal regions when encoding TBR items relative to young adults. As well, the results converge with ERP evidence for increased frontal activity in older adults during target processing when young adults' activation patterns are primarily parietal (Fabiani \& Friedman, 1995; for a review, see Friedman, 2003).

In contrast to prior investigations (Brandt et al., 2013; Gallant \& Dyson, 2016), emotional words did not influence cue-related potentials. One possible reason for this is that the emotional words were not high enough in emotional intensity (or arousal) to modulate ERP responses. As described in the General Method (page 38) the stimuli used in the current experiments were matched on mid-range levels of arousal to isolate the effects of valence. The aforementioned studies, in contrast, used sets of emotional words that were higher in arousal than the neutral baseline condition (Brandt et al., 2013; Gallant \& Dyson, 2016), and so it is possible that higher 
levels of stimulus intensity were needed to elicit an emotional response on cue-related ERPs. This is consistent with the dual competition model (Pessoa, 2009) described in Chapter 2, which suggests that the prioritization of emotional stimuli low in intensity has a relatively weak effect on cognitive control processes, whereas those high in intensity (e.g., threatening stimuli) can consume the top-down resources required for completing a cognitive task.

That emotion did not modulate cue-related ERPs may also help to explain why emotion did not influence directed forgetting performance in young or older adults. Initially, we (Gallant \& Yang, 2014) hypothesized that emotion would either enhance prioritization of TBR items and/or hinder suppression of TBF items, particularly for positive information in older adults. However, across several experiments, we have failed to observe such a pattern. A plausible explanation for this is that the directed forgetting manipulation consumed the cognitive resources required for older adults to engage in elaborative processing of positive items. As described in Chapter 1, the age-related positivity effect in cognition is rooted in top-down motivational goals to prioritize positivity as opposed to declines in the ability to process negative information (Knight et al., 2007; Mather \& Knight, 2005). This is supported by recent meta-analyses showing that the age-related positivity effect in memory is largest when encoding is unconstrained by task demands (Reed et al., 2014). The fact that emotion modulated word-related but not cue-related ERPs in the current experiment may provide support for this cognitive control account of the positivity effect. That is, it is possible that the task-related demands to selectively rehearse TBR items and suppress TBF items constrained older adults' ability to selectively allocate resources to positive information. As a result, the emotional effect on ERPs was wiped out when cues were presented. Although this interpretation is consistent with the cognitive control account of the 
positivity effect, it is important to note that it relies on interpreting a null effect and so should be viewed with caution.

This study was the first to examine how aging affects ERP correlates underlying directed forgetting of emotion. Together, the results replicate and add several important findings to this dissertation and the literature. First, we replicated our prior findings that both young and older adults can exert control over the encoding of information varying in emotional tone (Gallant \& Yang, 2014). Second, older adults' source monitoring was improved relative to Experiments 1 and 2, as indexed by reduced misattributions of positive TBF and new items to TBR sources, which may be linked to the repetition of stimuli in Experiment 3. The electrophysiological data further replicate the finding of distinct cue-related ERPs, including sustained frontal positivity in response to TBF cues and enhanced parietal activity following TBR cues. Finally, this study shows that older adults diverge from this pattern, with an increased reliance on frontal resources during encoding of TBR items, but age invariance in parietal responses to TBR cues. These data thus provide further support for the notion that the neural mechanisms underlying directed forgetting differ in young and older adults. 


\section{Chapter 8: General Discussion}

Prior literature has suggested that emotional biases change across the lifespan, with a motivational shift to prioritize positivity in later life (Mather \& Carstensen, 2005). Importantly, these emotional biases have been shown to influence cognition, guiding our attention and leaving a strong imprint in memory (Pessoa, 2009). However, this research has focused primarily on how emotion impacts attention and memory processes, with little information about how we might control such information via intentional forgetting in memory. Although typically perceived as a memory failure, forgetting has many adaptive benefits such as reducing interference from unwanted information and increasing the accessibility of relevant information. In the context of emotion, forgetting can aid memory performance and well-being by suppressing reminders or intrusions from emotional memories (Anderson \& Levy, 2009). In three experiments, this dissertation examined how aging and emotion influence this goal-directed process via cue- or value-based directed forgetting as well as its metacognitive components and neural correlates.

To briefly reiterate results, using a cue-based paradigm, Experiment 1 demonstrated age invariance in the ability to make metacognitive JOLs for TBR and TBF items that varied in emotion. However, older adults' source monitoring performance, an index of retrospective metacognition, was differentially impacted by emotion. Specifically, older adults were inclined to attribute or misattribute positive words to TBR cues, replicating the findings of Gallant and Yang (2014). Experiment 2 adopted a value-based directed forgetting paradigm, and the results showed that young and older adults could control encoding of words cued with either a gain $(+10)$ or loss $(-10)$ value, regardless of the emotional tone of words. But the source monitoring results again found that older adults have a bias to misattribute positive words to sources that are inherently higher in value (i.e., +10$)$. Finally, Experiment 3 found evidence for age differences in 
the neural mechanisms of directed forgetting. Relative to young adults, older adults showed a less distinct pattern of brain activity in response to TBR and TBF cues, driven by increased frontal activity during the encoding of TBR items. These results also showed no modulation of emotion on the ERP signatures of directed forgetting. The following sections further highlight the theoretical and empirical implications of this research.

\section{Cognitive Control of Emotional and Non-Emotional Memories \\ Young and older adults can intentionally forget emotional memories. Although}

typically perceived as a failure of memory, forgetting has many adaptive benefits. When

intentional, forgetting allows us to sift through the contents of memory so that the most relevant details remain primarily accessible. However, it is important to note that not all representations are treated equally during the stream of information processing: Some leave a stronger memory trace than others. For instance, a vast literature on emotionally enhanced memory suggests that emotional representations tend to assume priority in memory (Hamann, 2001; LaBar \& Cabeza, 2006; Pessoa, 2009). The degree to which we prioritize emotion has also been argued to vary depending on our age and time perspective. As contended by the socioemotional selectivity theory, as we get older, we shift from focusing on future-oriented goals to ones that are more emotionally meaningful, such as prioritizing positive well-being (Carstensen, 1995). Coinciding with this motivational shift, older adults are often found to focus less on negativity and more on positivity when processing emotional information relative to their young counterparts (Mather \& Carstensen, 2005; Reed \& Carstensen, 2012; Reed et al., 2014). As a result, older adults tend to show a memorial benefit for positive over negative information in attention and memory. Given the influence of aging and emotion on memory representations, it is important to understand how 
such factors would influence instances where forgetting is more favourable. The findings of this dissertation provide insights into this very question.

In Experiments 1 to 3, results consistently supported our previous findings that intentional forgetting of emotional information is attainable by both young and older adults, using either a cue-based (Experiments 1 \& 3; Gallant \& Yang, 2014) or value-based directed forgetting procedure (Experiment 2). Importantly, these results could not be attributed to the inability of the emotional stimuli to elicit an emotional response, as emotion did affect overall item recognition performance. Specifically, participants typically showed greater hit and false alarm rates for emotional items, suggesting that the emotional tone of words did modulate memory performance across both age groups. It is important to note that the increase in hits could be driven by a propensity to respond 'old' to emotional items during recognition, as was indicated by response biases that tended to be more liberal for emotional relative to neutral items across experiments. The ERP evidence from Experiment 3, however, supports the idea that processing during encoding was influenced by emotion because the LPP component was modulated by this factor when studying words (prior to cue presentation) in both age groups. This is consistent with previous studies showing enhanced LPPs to emotional stimuli (e.g., Brandt et al., 2013; Brown et al., 2013; Gallant \& Dyson, 2016; Hajack \& Olvet, 2008; Hauswald et al., 2010; Kisley et al., 2007; Olofsson et al., 2008; Wood \& Kisley, 2006; Yang et al., 2012), which are often associated with allocation of selective attention and rehearsal resources.

The lack of emotional modulation of the directed forgetting effect, however, suggests that participants could effectively override the enhancing effects of emotion to strategically control their encoding of TBR and TBF information. Evidence from ERPs again may provide some 
support for this notion. Although both age groups showed an effect of valence on their LPP amplitude prior to the cue, this effect did not persist after the cue was presented. Rather, ERPs following the cue showed the expected parietal positivity in response to TBR cues and frontal positivity in response to TBF cues, regardless of emotion, providing converging evidence for distinct ERP correlates underlying attempts to intentionally remember and forget (e.g., Brandt et al., 2013; Hauswald et al., 2010; Gallant \& Dyson, 2016; Paller, 1990; Paz-Caballero \& Menor,1999; Paz-Caballero et al. 2004; van Hooff \& Ford, 2011; Yang et al., 2012). According to this literature, these divergent patterns of ERPs may support a two-factor account of directed forgetting in which selective rehearsal and active mechanisms are engaged to follow the TBR and TBF instructions, respectively (e.g., Anderson \& Hanslmayr, 2014; Basden \& Basden, 1996; Zacks et al., 1996). As such, results from Experiment 3 may suggest that, regardless of age, these mechanisms can be effectively applied to emotional stimuli that are relatively low in intensity.

As mentioned in Chapter 2, although these findings cannot lend direct support to the socioemotional selectivity theory and the age-related positivity effect, the results are consistent with a cognitive control account of this theory (Carstensen, 1995). According to this account, older adults require high levels of cognitive control to direct information processing resources toward positive information in the service of emotional goals (Mather \& Knight, 2005). In other words, the positivity effect is thought to be the product of controlled, goal-directed processing. In the current experiments, it is thus likely that the goal-directed mechanisms required to engage in directed forgetting overrode older adults' motivation to elaborate on positive stimuli (Reed \& Carstensen, 2012). As a result, young and older adults showed similar directed forgetting performance for emotional words. Thus, these findings may add to the literature by highlighting another boundary condition for the age-related positivity effect: item-directed forgetting. 
Altogether, these results suggest that both young and older adults can exert control over emotional memories, despite evidence for emotionally enhanced memory (Hamann, 2001; LaBar \& Cabeza, 2006) and age-related positivity effects in memory (Mather \& Carstensen, 2005). This importantly replicates not only our own work (Gallant \& Yang, 2014) and recent directed forgetting studies (Berger et al., 2016), but other studies using similar methods for examining memory control, including the think/no-think task (Murray et al., 2011) and the value-directed remembering procedure (Eich \& Castel, 2016). That we can maintain cognitive control over emotion is remarkably adaptive to daily functioning. It allows us to maintain goal-directed thought, limits our ability to access unwanted memories, and facilitates our ability to control reminders and/or responses to emotional memories. In turn, this enhances not only future learning and memory performance but also the ability to regulate emotion and positive wellbeing (Ochsner \& Gross, 2005).

Age-related similarities and differences in directed forgetting. It is important to note that there were differences in how age influenced directed forgetting across experiments. Most notably, an age-related reduction in the behavioural directed forgetting effect was only observed in Experiment 3, the design of which was most similar to that of our original study (Gallant \& Yang, 2014). In this study, the modified remember/know procedure also indicated that the contribution of recollection-based processes to directed forgetting may have been reduced in older relative to young adults. Basden and Basden (1996) have suggested that the greater contribution of recollection-based processes to directed forgetting likely reflects increased distinctive processing of TBR relative to TBF items. In Experiment 3, older adults may thus have had more difficulty encoding contextual features of TBR items during the study phase, which is consistent with evidence for an age-related decline in the ability to recollect episodic information 
during retrieval (e.g., Anderson et al., 2008). In turn, older adults' memory for TBR items may have been weaker, making them more difficult to retrieve during recognition.

Contrasting with the findings of Experiment 3, age equivalence in directed forgetting was observed in Experiments 1 and 2, which suggests that something about the design of these experiments either enhanced older adults' directed forgetting or reduced that of young adults. In comparing the results of Experiment 1 with Gallant and Yang (2014), it appears that the null effect of age in Experiment 1 may be attributable to reduced directed forgetting in young adults. In Experiment 1, young adults remembered $77 \%$ of TBF items relative to $62 \%$ (recognition of TBR items remained the same) in the study of Gallant and Yang (2014). Older adults' recognition of TBF items increased only slightly across experiments with $76 \%$ in Experiment 1 and $71 \%$ in Gallant and Yang (2014). In explaining these results, it is possible that the JOL promoted distinctive processing of stimuli by requiring participants to judge the likelihood of later remembering the stimuli. As such, the JOL may have either enhanced processing of TBF items or interfered with the ability to engage the appropriate mechanisms for suppressing TBF items which, according to the ERP results of Experiment 3, are typically evoked immediately following a TBF cue. While this explanation is of course speculative, the results nevertheless fall in line with other studies that have shown age equivalence in directed forgetting when levels of processing manipulations are implemented (e.g., Berger et al., 2016; Gamboz \& Russo, 2002).

With regard to Experiment 2, the age invariance in directed forgetting seemed instead to be driven by an increased magnitude of directed forgetting in the older group when compared with that of Gallant and Yang (2014). Although older adults in Experiment 2 showed overall lower recognition relative to that of the former experiment (67\% in Experiment 2 vs. $76 \%$ in Gallant \& Yang, 2014), the overall magnitude of directed forgetting (i.e., TBR - TBF items) in 
older adults was enhanced by nearly $10 \%$ in Experiment 2 relative to Gallant and Yang (20\% vs. $11 \%$ difference, respectively). Although these comparisons are interesting, again it is important to note that they are speculative as no statistical cross-experiment comparisons were conducted. However, the lack of age differences in Experiment 2 is also consistent with findings in the value-directed remembering literature, which uses numeric values to induce selective memory encoding (e.g., Castel, Benjamin, Craik, \& Watkins, 2002; Castel, Farb, \& Craik, 2007; Eich \& Castel, 2016). For example, Eich and Castel (2016) recently found that whereas young adults tend to recall more items overall, both age groups were equivalent in their ability to prioritize high-value items and suppress low-value items. Coupled with the value-directed remembering literature, the results of Experiment 2 may thus suggest that, relative to TBR and TBF cues, numeric point values are a more salient cue for inducing older adults to selectively encode and suppress materials. Most intriguingly, these results suggest that intentional forgetting performance in aging may be improved by simple changes in cue type.

Shifting the focus from behaviour to brain, the ERP results further highlight important age differences in the neural correlates of intentional forgetting. Both age groups showed the expected parietal positivity in response to TBR cues, which is thought to reflect the engagement of selective rehearsal mechanisms for encoding these items (e.g., Brandt et al., 2013; Gallant \& Dyson, 2016; Hauswald et al., 2010; Paz-Caballero et al., 2004). In young adults, TBF cues evoked enhanced frontal positivity relative to TBR cues, possibly driven by the engagement of active mechanisms for suppressing these items (Anderson \& Hanslmayr, 2014). However, during early and middle time windows, older adults did not show this differentiation in frontal positivity (i.e., TBF > TBR). In fact, this differentiation did not appear until the final epoch $(600-800 \mathrm{~ms})$. Relative to young adults, they showed increased frontal positivity in response to TBR cues in the 
$350-550 \mathrm{~ms}$ time window, which was directly correlated with their subsequent recognition

performance. These ERP findings are consistent with Rizio and Dennis' (2014) fMRI

investigation, which found increased recruitment of the PFC during encoding attempts of TBR items in older but not young adults that was predictive of subsequent memory.

On the one hand, it could be argued that these increased patterns of activity reflect agerelated dedifferentiation in which older adults show reduced selectivity in the recruitment of specialized neural mechanisms when completing a behavioural task. As a result, the aging brain exhibits an increased amount of extraneous noise (Grady, 2012). However, the fact that an association between older adults' increased brain activity and recognition occurred counters the idea that this activity is simply noise and instead may fit the criteria for successful compensation, in which additional activity supports behavioural performance (Cabeza \& Dennis, 2012; Grady, 2008, 2012). Put differently, this pattern of activity may represent an age-related reorganization of brain activity in which anterior mechanisms are recruited to facilitate successful performance. As proposed by Friedman (2003), one possibility is that these age differences in neural recruitment reflect an increased tendency in older adults to continually consult target stimuli in working memory to ensure that each item is successfully encoded before presentation of the next item. In turn, older adults' frontal activity is increased to support these processes when compared to their young counterparts who may require fewer resources to encode information.

\section{Metacognitive Aspects of Intentional Forgetting of Emotional Information}

This dissertation also provided insights on the metacognitive aspects of intentional forgetting, including the ability to make inferential judgments about the learning and retrieval of TBR and TBF words. To briefly reiterate, metacognition can be thought of as awareness of our own cognitive capacities. According to Metcalfe and Dunlosky (2008), metacognition 
encompasses the "processes and structures whereby people examine the content of their memories, either prospectively or retrospectively, and make judgments or commentaries about them". Metacognition is thought to be a critical process underlying effective cognition. According to Hertzog and Dunlosky (2011), to achieve control over one's cognition, we must be able to actively monitor ongoing cognitive processes to decide whether a specific goal is being met. For example, older adults learning how to identify the symptoms of a stroke using the F.A.S.T. acronym (i.e., face, arms, speech, and time; Heart and Stroke Foundation, 2017) will need to monitor whether they can accurately retrieve their knowledge of which letter corresponds to which symptom so that they can make the decision on whether to spend more time studying the information. In this way, the association between monitoring and control is reciprocal (Hertzog \& Dunlosky, 2011; Nelson \& Narens, 1990): By monitoring our cognitions, we can implement strategies to improve our cognitive processing.

As previously described, metacognitive monitoring of learning, as inferred from JOLs, tends to be spared in aging (Hertzog \& Dunlosky, 2011). Accordingly, Kuhlmann and Touron (2011) have shown that older adults, like young adults, can use extrinsic cues about differences in the memorability of information to guide their JOLs. Evidence from Experiment 1 fell nicely in line with these results. Both age groups showed enhanced JOLs for emotional relative to neutral information as well as for TBR relative to TBF items. The resolution of JOLs for both young and older adults was also above chance for TBR items, but not for TBF items, which is consistent with the findings of Friedman and Castel's (2011) study with young adults. Together, these results imply that whereas participants have good awareness of their ability to intentionally remember, they are less able to predict their ability to forget information. Visual comparison of TBF JOLs and recognition suggests that this may be due to some overconfidence in the ability to 
forget. Nevertheless, the modulation of JOLs in response to cues suggests some awareness of the dynamics of forgetting that seems resistant to aging.

The results from Experiment 1 also showed that older adults' JOLs were sensitive to the benefits of emotion regardless of memory cue, as higher JOLs were attributed to emotional (both positive and negative) over neutral items and corresponded to subsequent recognition. These results contrast with the findings of Tauber and Dunlosky (2012), who compared young and older adults on their JOLs during a recall task for positive, negative, and neutral words. Their findings indicated that whereas young adults showed higher JOLs and relative accuracy for emotional (positive and negative) over neutral words, older adults only showed this pattern for negative and neutral words but not for positive, suggesting that older adults' JOLs were insensitive to the benefits of positive emotion. A speculation for this divergence of results is that the explicit cues to remember and forget in Experiment 1 helped older adults to better direct their encoding resources and thus JOLs toward emotional information. In turn, JOLs and hit rates were higher for emotional material, leading to better resolution of JOLs for these items. In contrast, Tauber and Dunlosky's (2012) experiment did not include any sort of cuing manipulation, as all words were fair game for subsequent memory. All in all, the results of Experiment 1 extend this literature by showing that older adults are just as able as young adults to adjust their metacognitive JOLs according to TBR and TBF cues and emotional items.

Experiments 1-3 also examined the impact of emotion and aging on source monitoring, which is comprised of the processes through which we make retrospective judgments about the origins of our memories (Mitchell, 2016). According to the source monitoring framework (Johnson et al., 1993) on the one hand, these judgments can be based on characteristics of the information entering memory (e.g., cognitive operations applied during learning), which become 
bound together to form a rich representation that facilitates later recall of the source (Johnson et al., 1993). On the other hand, we often infer the source of information based on our beliefs or general knowledge about what a memory from a source should be like. As outlined by Kuhlmann and Bayen (2016), reliance on such metacognitive knowledge can help us to infer the correct source of information when memory for source features is lacking, such as when we remember information but fail to remember where or who we learned that information from. Reliance on such information may become particularly important in aging, as the ability to form bound representations of items and their contextual features becomes more difficult as we get older (Old \& Naveh-Benjamin, 2008). However, in some cases, reliance on this knowledge may lead to systematic errors of misattribution.

In the current set of experiments, young and older adults' source monitoring was differentially influenced by valence and cue. In line with prior findings (e.g., Thompson et al., 2011), young adults' source attributions of TBR and TBF items were relatively good (i.e., usually around $70-75 \%$ accurate), regardless of valence or cue. In contrast, older adults consistently showed a source memory advantage for positive TBR relative to positive TBF items. Drawing on theories of socioemotional selectivity and age-related positivity effects (Carstensen, 1995; Mather \& Carstensen, 2005), these results may suggest that older adults are more readily able to bind features of items that are consistent with their goals. In this case, binding a TBR cue with a positive word would be more consistent with their emotion-focused goals to emphasize positivity than with binding a cue to forget with a positive word. This pattern of findings is consistent with evidence from other studies that have found an advantage in older adults' memory for emotional sources (May et al., 2005) and a better ability to remember the emotional as opposed to perceptual characteristics of memories (Hashtroudi et al., 1990). Consistent with 
the age-related positivity effect, this seems to suggest that older adults may devote more elaborative processing to these items during encoding, enhancing their source memory.

Although the above explanation suggests that older adults' source memory advantage for positive TBR items may be driven by encoding processes, it is important to note that the current experiments provide evidence against this idea. In Experiment 1, older adults' JOLs for TBR and TBF items did not vary as a function of emotion. If older adults devoted more effort to encoding positive TBR items, enhancing binding of the word and cue, then it is likely that JOLs would have been differentially higher for these items relative to the other conditions, yet this was not the case as JOLs were similar for positive and negative items across cue conditions. As indicated by Experiment 3, older adults' source memory for positive TBR items was also not accompanied by greater conscious recollection for contextual detail, which further suggests that these items may not have received distinctive processing above and beyond the other conditions.

The ERP evidence in Experiment 3 also counters an encoding-based account as older adults' word-related LPPs were not greater for positive over neutral words; only a reduction in the LPPs to negative words was observed. Valence also did not modulate the cue-related parietal activity that is argued to reflect strategic allocation of attentional resources. If older adults were engaging in elaborative processing for positive TBR items, it is likely that ERPs during this time window would have been selectively enhanced for these items. However, no valence-specific influence was observed on cue-related ERP activity. Altogether, the current findings cannot attest to the notion that older adults' encoding was specifically enhanced for positive over negative or neutral TBR items during encoding. Rather, the evidence seems to favour a recognition-based account in which older adults have a bias to attribute positive information to sources that are higher in relevance (i.e., TBR cues) or value (i.e., +10 cues). 
Evidence for the recognition-based account may be supported by older adults' tendency to misattribute positive TBF and new words to TBR sources. This contrasts with what would be expected if older adults had relied on their knowledge of cognitive operations about TBR and TBF sources during the source monitoring task. If they are using such metacognitive knowledge about differences in cognitive operations between cues, participants should show a tendency to attribute new items that they guessed as old to the cue with low item memorability, as these new items would not be accompanied by a rich memory trace. This was indeed the case for young adults, regardless of valence, as they attributed more new words to TBF relative to TBR sources. In contrast, older adults showed this attribution pattern (i.e., TBF $>$ TBR) only for new words that were negative or neutral, whereas the opposite was observed for positive items in Experiments 1 (i.e., $\mathrm{TBR}>\mathrm{TBF}$ ) and 2 (i.e., $\mathrm{TBR}=\mathrm{TBF}$ ). As proposed in Experiment 2, it is possible that, due to an age-related shift to prioritize positive information, older adults have a preference to attribute positive items to sources that are in line with their goals. This may reflect a strategy or means to compensate for poor source memory in which older adults evaluate which source an item should belong to by relying on their own beliefs (Kuhlmann \& Bayen, 2016). This may benefit their performance for instances where the item and source match (positive TBR items) but impair performance when the item and source are incongruent (positive TBF items).

\section{Limitations}

These experiments were not without limitations. First, the approach for sampling young and older adults may be perceived as a limitation. In Experiments 1-3, the older group had a wider age range (20 years) than the young group ( $<10$ years), which is a common practice in aging literature. However, it is well-documented that aging is accompanied by increases in performance variability both within and between participants (Morse, 1993). As such, by not 
placing an upper age limit on the older group, the degree of variability in the older sample may have been elevated relative to that in the young sample. To reduce such variability and increase effect size, future studies may consider using similar age ranges across young and older groups. Or, to gain a more comprehensive understanding of age differences in directed forgetting, another approach may be to compare young adults with both young-old (60-74 years) and oldold adults (75-89 years; for a similar approach, see Berger et al., 2016). While on the topic of the older adult sample, it is worth noting that these participants were highly educated across experiments, with an average of 15 to 17 years of education. High levels of education in older adults have been associated with cognitive reserve and better performance on cognitive tests (Stern, 2002), and so it is possible that the findings acquired with the current older samples may not be applicable to the general population.

A second limitation is that the current dissertation only focused on ERP correlates of encoding-related behaviour and thus cannot speak to which processes are involved in the retrieval or source monitoring of TBR and TBF items between age groups. Typically, ERPs during recognition tasks reveal the parietal 'old/new effect', which is characterized by positivegoing ERP amplitudes over parietal sites and is thought to reflect the recollection of old relative to new items (for a review, see Rugg \& Curran, 2007). In directed forgetting, there is evidence that the correctly retrieved TBR cues elicit the parietal old/new effect whereas forgotten TBF items elicit a reversed negative-going old/new effect, thought to reflect effective inhibition of encoding and retrieval processes (Nowicka, Jednoróg, Wypych, \& Marchewka, 2009). In the context of aging, several studies have shown that the old/new ERP signature of recollection tends to be diminished in older adults (for a review, see Friedman, 2013). As such, it is likely that age differences would have also emerged in ERPs associated with the retrieval of TBR and TBF 
items. However, Experiment 3 was limited in the number of observations for conducting such an analysis as there were only 60 old items ( $30 \mathrm{TBR}, 30 \mathrm{TBF}$ ) and 60 new items in the recognition task (10 items per valence and cue condition). According to Luck (2014), a minimum of 30 to 40 trials per condition is recommended for examining large components such as the P300 family. Once factoring in subsequent recognition performance, this would have left too few items per cell in Experiment 3 to garner enough power to detect differences in ERP responses. Future research is thus required to further delineate age differences in the retrieval-based ERP correlates of directed forgetting.

A third limitation concerns the stimuli used in this experiment. In the current set of experiments, any time that lists were divided to form cue or valence conditions or lists of old and new stimuli, the lists were matched on the degree of emotional arousal, word frequency, and word length. However, previous research has suggested that the degree of semantic relatedness between emotional words within a list may contribute to emotionally enhanced memory. For instance, across three experiments, Talmi and Moscovitch (2004) found that emotional words only conferred a benefit in memory when compared against random neutral words that were not semantically related. When neutral words were organized into categories to increase their semantic relatedness, the advantage of emotion in memory was no longer observed. These results imply that the semantic relatedness among emotional items may underlie their benefit in memory in essence providing a retrieval cue not available for the neutral words. In the current experiment, it seems unlikely that semantic relatedness among the emotional items played a role in the observed effects, otherwise young adults would have also shown an effect of valence in their source monitoring, for example. Instead, the effect was specific to older adults. Thus, even 
if emotional words were higher in semantic relatedness than neutral words, it seems unlikely that this played a role in the observed effects.

\section{Future Directions}

There are many outstanding questions with regard to the findings of this dissertation. Coupled with the results from our initial study (Gallant \& Yang, 2014), Experiments 1-3 demonstrated that young and older adults can strategically control the encoding of TBR and TBF (or high- vs. low-value) emotional information. However, as previously mentioned, this study primarily focused on the effects of emotional valence, given research that the age-related positivity effect is most likely to be observed for information that is nonarousing but high in valence (Kensinger, 2008). As such, a question to be addressed is how older adults' directed forgetting would fare in the face of emotional arousal. As illustrated by Kensinger and Corkin (2004), the detection of emotional arousal is thought to rely on automatic bottom-up processes, relative to the controlled top-down processes that enhance attention and memory for emotional valence. In the context of directed forgetting, top-down processes are further required to actively suppress TBF representations and selectively process TBR items. As previously discussed, the reason that we failed to find emotional effects in older adults' directed forgetting was likely that the top-down processes needed to implement the memory cues (i.e., TBR or TBF) overrode older adults' goals to prioritize positivity. When considering the impact of emotional arousal on directed forgetting, the question becomes whether older adults can reconcile competition between top-down and bottom-up processes. In other words, top-down processes would be required to implement the memory cue, whereas stimuli high in emotional arousal would activate bottom-up information processing. Literature with young adults has shown a reduced ability to suppress highly arousing negative TBF images (e.g., Hauswald et al., 2010; Nowicka et al., 
2010) and words (e.g., Gallant \& Dyson, 2016; Yang et al., 2015). Given that older adults often show activation toward emotional arousal like that of young adults (Kensinger, 2008), one might hypothesize that their directed forgetting performance for such information would follow suit.

The current set of experiments can also only speak to the immediate effects of emotion on directed forgetting and so future research should examine how amenable these effects (or lack thereof) are to the passage of time. Previous research has suggested that emotion slows forgetting via enhanced memory consolidation (e.g., Sharot \& Phelps, 2004). In line with this idea, Sharot and Yonelinas (2008) demonstrated that recollection-based memory for emotion is enhanced after a 24-hour retention interval, whereas no such benefits were observed in an immediate test. In addition, studies have shown that sleep may prioritize the effect of top-down, goal-directed cues on subsequent memory by facilitating consolidation of such information (e.g., Bennion, Payne, \& Kensinger, 2016). If this is the case then, relative to neutral items, it is possible that emotional TBR items and incidentally encoded emotional TBF items may confer a memorial benefit following a 24 -h time window in which participants are allowed additional consolidation time during a night of sleep. As such, it remains an open question as to whether an emotional effect on directed forgetting may be observable on a delayed relative to an immediate test of memory and how this might differ across age groups.

Finally, a question for future research is whether young and older adults can improve their metacognitive predictions for information that they must suppress. This is important: As previously described, metacognitive monitoring is an integral aspect of learning, allowing us to track online performance and adjust our learning strategies as needed. The results of Experiment 1 suggest that both young and older adults' JOLs were sensitive to extrinsic cues as they were higher for TBR than TBF cues. These JOLs for TBR items were also predictive of their 
subsequent recognition whereas JOLs for TBF items were not, which seemed to be due to some overconfidence in the ability to suppress TBF items. Prior research has indicated that it is possible to train older adults to monitor their learning using JOLs as a means to adapt learning strategies which ultimately improved their ability to learn and remember the association between pairs of stimuli (e.g., Bailey, Dunlosky, \& Hertzog, 2010; for a review, see Hertzog \& Dunlosky, 2011). Following from the results of Experiment 1, an open question is whether young and older adults could be trained to improve monitoring of their ability to learn TBR information and to suppress TBF information via JOLs. As a byproduct of such an intervention, improvements in the ability to regulate cognitive control processes during directed forgetting may also be observed.

\section{Conclusions}

In closing, the findings of this dissertation have important implications for theories surrounding socioemotional selectivity (Carstensen, 1995) and age-related positivity biases (Reed \& Carstensen, 2012), suggesting that older adults can control attention and memory for positive information. Extending previous literature on metacognition and aging, this dissertation also demonstrated age invariance in the ability to prospectively monitor encoding of TBR and TBF emotional information via judgments of learning. In contrast, older adults' ability to use metacognitive knowledge when retrospectively attributing TBF and new words to sources was

disrupted by positive information. These results may call into question the adaptive benefit of the age-related positivity effect. While prioritizing emotional goals over others may serve to enhance positive well-being in aging, there may be instances where that information can cloud performance and lead to systematic errors in certain types of cognitive processing. 
Finally, the results further our understanding of how the neural correlates associated with directed forgetting are influenced by aging and emotion by showing that (1) emotional information low in arousal does not appear to modulate the ERP signatures typically associated with TBR and TBF cues, and (2) older adults may recruit compensatory activity in frontal regions to facilitate their encoding of TBR information. Consistent with prior research (Rizio \& Dennis, 2014), this highlights the fact that different patterns of brain activity underlie the control of TBR and TBF memories in young and older adults. Altogether, these findings add to our knowledge of how "cold" executive control processes interact with the "hot" emotion functions of the aging brain, suggesting that the ability to exert control over emotional memories is intact in later life. 


\title{
Appendices
}

\section{Appendix I: Consent Form for Older Adults in Experiment 1}

\author{
Ryerson \\ University \\ Ryerson University, Department of Psychology \\ Consent Agreement
}

Please read this consent form so that you understand what your participation involves. Before you consent, please ask any questions you may have with regards to your participation.

\section{STUDY TITLE: Effects of age and emotion on memory predictions}

INVESTIGATORS: This research is being conducted by Sara Gallant ( $\mathrm{PhD}$ candidate) and Dr. Lixia Yang (Associate Professor) from the Department of Psychology at Ryerson University.

PURPOSE OF THE STUDY: The purpose of this cognitive psychology study is to assess how aging and emotion affects our ability to accurately predict memory performance. Thirty-six older and 36 young adults will be invited to participate in this study.

DESCRIPTION OF THE STUDY: If you decide to participate, your visit will last 60 minutes. After signing the consent form, you will be asked to complete a learning task during which you will study a series of words that you will need to either remember or forget for a later recognition memory test. After studying each item, you will provide a prediction concerning the likelihood that you will remember that item on the later recognition task. During the recognition task, you will be shown the words you learned again as well as several new words and you will be asked to identify which items you learned and which are new. Following the learning task, you will be asked to complete questionnaires that will assess your mood and anxiety level. As well, some basic demographic and health information will be collected (e.g., age, gender, education level, medication use, health conditions). At the end of the session, you will receive your incentive, regardless of whether all aspects of the experiment have been completed.

WHAT IS EXPERIMENTAL IN THIS STUDY: From a technical or procedural point of view, part of this study is considered "experimental" because the procedure allows us to examine the impact of one variable (the "independent variable"; e.g., aging) on another variable (the "dependent variable"; e.g., memory). More information about the independent and dependent variables will be provided at the end of the session.

RISKS: This is a minimal risk study. However, minor risks may include discomfort from the questions being asked due to their personal nature. Participants may choose to refuse participation in any aspect of the research (e.g., responding to questionnaire items). Any discomfort is expected to be temporary and not greater than what you might experience in a typical day. If any aspect of this study makes you uncomfortable, you may temporarily or permanently discontinue participation without penalty and you will still be fully compensated. 
BENEFITS: We hope the study will benefit society and science by providing a greater understanding of how certain mental abilities change as we age. Participants will receive no direct benefit although information gained from the study may improve their knowledge on the topic of cognition and aging. When the session is over, we will describe the purpose and hypotheses of the study to you in more detail.

CONFIDENTIALITY: You will not put your name on the questionnaires. You will be asked to sign only this consent form, and both the consent form and the background questionnaire will be filed separately from your other questionnaires. Your responses in this research will be confidential so there will be no way of linking your responses with your identity. Data from this study will be held in a locked lab room for up to ten years and on a password protected computer to which only investigators and associated personnel will have access. Should the data be used for any secondary analyses, you will be made aware. After this period, the data will be destroyed.

INCENTIVES TO PARTICIPATE: You will receive \$12 for your participation.

VOLUNTARY NATURE OF PARTICIPATION: Participation is voluntary. Your choice of whether to participate has no bearing on your past, present, or future relationship with Ryerson University. If you participate, you are free to withdraw your consent and stop participation at any time without penalty or loss of benefits to which you are allowed. Should you withdraw from the study, you will still receive your token of appreciation.

QUESTIONS: If you have any questions, please ask now otherwise you may contact: Sara Gallant (ㄱallan@psych.ryerson.ca) or Dr. Lixia Yang (lixiay@psych.ryerson.ca). If you have questions regarding your rights as a participant, you may contact the Ryerson University Research Ethics Board c/o Office of the Vice President, Research and Innovation, Ryerson University 350 Victoria Street Toronto, ON M5B 2K3, 416-979-5042.

AGREEMENT: Your signature indicates that you have read this form and have had a chance to ask any questions you have. It also indicates that you agree to participate and understand that you can withdraw at any point. You have been given a copy of this agreement. By signing, you are aware that you are not giving up any of your legal rights.

$\overline{\text { Name of Participant (please print) }} \overline{\text { Signature of Participant }} \quad \overline{\text { Date }}$

$\overline{\text { Signature of Investigator }} \quad \overline{\text { Date }}$




\title{
Appendix II: Consent Form for Young Adults in Experiment 1
}

\author{
Ryerson
University \\ Ryerson University, Department of Psychology \\ Consent Agreement
}

Please read this consent form so that you understand what your participation involves. Before you consent, please ask any questions you may have with regards to your participation.

\section{STUDY TITLE: Effects of age and emotion on memory predictions}

INVESTIGATORS: This research is being conducted by Sara Gallant ( $\mathrm{PhD}$ candidate) and $\mathrm{Dr}$. Lixia Yang (Associate Professor) from the Department of Psychology at Ryerson University.

PURPOSE OF THE STUDY: The purpose of this cognitive psychology study is to assess how aging and emotion affects our ability to accurately predict memory performance. Thirty-six older and 36 young adults will be invited to participate in this study.

DESCRIPTION OF THE STUDY: If you decide to participate, your visit will last 60 minutes. After signing the consent form, you will be asked to complete a learning task during which you will study a series of words that you will need to either remember or forget for a later recognition memory test. After studying each item, you will provide a prediction concerning the likelihood that you will remember that item on the later recognition task. During the recognition task, you will be shown the words you learned again as well as several new words and you will be asked to identify which items you learned and which are new. Following the learning task, you will be asked to complete questionnaires that will assess your mood and anxiety level. As well, some basic demographic and health information will be collected (e.g., age, gender, education level, medication use, health conditions). At the end of the session, you will receive your incentive, regardless of whether all aspects of the experiment have been completed.

WHAT IS EXPERIMENTAL IN THIS STUDY: From a technical or procedural point of view, part of this study is considered "experimental" because the procedure allows us to examine the impact of one variable (the "independent variable"; e.g., aging) on another variable (the "dependent variable"; e.g., memory). More information about the independent and dependent variables will be provided at the end of the session.

RISKS: This is a minimal risk study. However, minor risks may include discomfort from the questions being asked due to their personal nature. Participants may choose to refuse participation in any aspect of the research (e.g., responding to questionnaire items). Any discomfort is expected to be temporary and not greater than what you might experience in a typical day. If any aspect of this study makes you uncomfortable, you may temporarily or permanently discontinue participation without penalty and you will still be fully compensated. 
BENEFITS: We hope the study will benefit society and science by providing a greater understanding of how certain mental abilities change as we age. Participants will receive no direct benefit although information gained from the study may improve their knowledge on the topic of cognition and aging. When the session is over, we will describe the purpose and hypotheses of the study to you in more detail.

CONFIDENTIALITY: You will not put your name on the questionnaires. You will be asked to sign only this consent form, and both the consent form and the background questionnaire will be filed separately from your other questionnaires. Your responses in this research will be confidential so there will be no way of linking your responses with your identity. Data from this study will be held in a locked lab room for up to ten years and on a password protected computer to which only investigators and associated personnel will have access. Should the data be used for any secondary analyses, you will be made aware. After this period, the data will be destroyed.

INCENTIVES TO PARTICIPATE: You will receive one participation credit to use towards your PSY102/202 course. If you prefer to 'walk through' the study (i.e., to observe the research but not provide any personal data), you will still be given the one credit assuming you have not already received the maximum allotted for research participation. Please be advised that a 'walk through' is not a short version of the study, but rather takes you through the full session.

VOLUNTARY NATURE OF PARTICIPATION: Participation is voluntary. Your choice of whether to participate has no bearing on your past, present, or future relationship with Ryerson University. If you participate, you are free to withdraw your consent and stop participation at any time without penalty or loss of benefits to which you are allowed. Should you withdraw from the study, you will still receive your token of appreciation.

QUESTIONS: If you have any questions, please ask now otherwise you may contact: Sara Gallant (s7gallan@psych.ryerson.ca) or Dr. Lixia Yang (lixiay@psych.ryerson.ca). If you have any questions regarding your involvement in the SONA participant pool, please contact thepool@psych.ryerson.ca. If you have questions regarding your rights as a participant, contact the Ryerson University Research Ethics Board c/o Office of the Vice President, Research and Innovation, Ryerson University 350 Victoria Street Toronto, ON M5B 2K3, 416-979-5042.

AGREEMENT: Your signature indicates that you have read this form and have had a chance to ask any questions you have. It also indicates that you agree to participate and understand that you can withdraw at any point. You have been given a copy of this agreement. By signing, you are aware that you are not giving up any of your legal rights.

$\overline{\text { Name of Participant (please print) }} \overline{\text { Signature of Participant }} \quad \overline{\text { Date }}$

$\overline{\text { Signature of Investigator }} \quad \overline{\text { Date }}$ 


\section{Appendix III: Debriefing form for Experiment 1}

\section{Ryerson University}

Debriefing: Age differences in predictions of memory for emotional information

Metamemory can be defined as knowledge or awareness of one's own memory ability (Castel, McGillivray, \& Friedman, 2012). Such awareness is important as being able to accurately predict what we will remember later allows us to adapt learning strategies in order to improve performance. However, there are some instances where it may be more beneficial to forget previously learned information in favour of more updated or recent information.

In this study, we examined age differences in the accuracy of predictions for memory of relevant ('to-be-remembered') or irrelevant ('to-be-forgotten') words. We also looked at whether the emotional status of the word (i.e., positive, negative, or neutral) would impact these predictions and how this would relate to memory performance. Prior research has suggested an age-related positivity bias in which older adults show a preference for positive over negative information in attention and memory, whereas young adults show an equal focus on positive and negative stimuli or a bias toward negative (Mather \& Carstensen, 2005). Considering this, we expect age differences in predictions across the emotional conditions such that older adults indicate higher likelihood of remembering positive than negative or neutral words relative to their young counterparts. We also hypothesize that predictions will be sensitive to the memory cues (i.e., to-be-remembered or to-be-forgotten), with higher predictions of remembering for tobe-remembered than for to-be-forgotten words, an effect consistent with that found in young adults by Friedman and Castel (2011). However, as some older adults may hold certain beliefs about their memories based on experience or age-related memory stereotypes, it is possible they might underestimate the degree to which they will remember to-be-remembered items and - to an even greater extent - to-be-forgotten items (i.e., overestimating the rate of forgetting).

Overall, we hope that this study will provide an understanding of how awareness or knowledge of our own mental functions change across the lifespan.

For more information regarding this study, you may consult the following sources:

Friedman, M. C., \& Castel, A. D. (2011). Are we aware of our ability to forget? Metacognitive prdictions of directed forgetting. Memory and Cognition, 39, 1448-1456.

Mather, M. \& Carstensen, L. L. (2005). Aging and motivated cognition: The positivity effect in attention and memory. Trends in Cognitive Sciences, 9(10), 496-502.

Thank you for your participation! Please do not hesitate to ask questions now or later. You can contact the principal investigator, Sara Gallant (s7gallan@psych.ryerson.ca) or Dr. Lixia Yang (lixiay@psych.ryerson.ca). 


\title{
Appendix IV: Consent Form for Older Adults in Experiment 2
}

\author{
Ryerson
University \\ Ryerson University, Department of Psychology \\ Consent Agreement
}

Please read this consent form so that you understand what your participation involves. Before you consent, please ask any questions you may have with regards to your participation.

\section{STUDY TITLE: The influence of value on memory}

INVESTIGATORS: This research study is being conducted by Sara Gallant ( $\mathrm{PhD}$ candidate) and Dr. Lixia Yang (Associate Professor) from the Department of Psychology at Ryerson University.

PURPOSE OF THE STUDY: The purpose of this cognitive psychology study is to assess how we perceive and remember information that varies in high- vs. low-value. Twenty-four older and 24 young adults will be invited to participate in this study.

DESCRIPTION OF THE STUDY: If you decide to participate, your visit will last approximately 60 minutes. After signing the consent form, you will be asked to complete a task during which you will study a series of different types of words. Some of these words will be designated as high-value items (+10 points) while others will be low-value items (-10 points). Your goal is to remember as many high-value items while suppressing the low-value items. Following the study phase, a recognition task will be administered in which you will be asked to indicate whether you previously studied the word or if it is new. This design allows us to determine your ability to prioritize high-value information in attention and memory while deprioritizing low-value information. After the computer task, you will be asked to complete questionnaires on your mood, anxiety level, demographic and health information (e.g., age, gender, education level, medication use, health condition). At the end of the session, you will receive your incentive, regardless of whether all aspects of the study have been completed.

WHAT IS EXPERIMENTAL IN THIS STUDY: From a technical or procedural point of view, part of this study is considered "experimental" because the procedure allows us to examine the impact of one variable (the "independent variable"; e.g., aging) on another variable (the "dependent variable"; e.g., memory). More information about the independent and dependent variables will be provided at the end of the session.

RISKS: This is a minimal risk study. However, minor risks may include discomfort from the questions being asked due to their personal nature. Participants may choose to refuse participation in any aspect of the research (e.g., responding to questionnaire items). Any discomfort is expected to be temporary and not greater than what you might experience in a typical day. If any aspect of this study makes you uncomfortable, you may temporarily or permanently discontinue participation without penalty and you will still be fully compensated. 
BENEFITS: We hope the study will benefit society and science by providing a greater understanding of how certain mental abilities change as we age. Participants will receive no direct benefit although information gained from the study may improve their knowledge on the topic of cognition and aging. When the session is over, we will describe the purpose and hypotheses of the study to you in more detail.

CONFIDENTIALITY: You will not put your name on the questionnaires. You will be asked to sign only this consent form, and both the consent form and the background questionnaire will be filed separately from your other questionnaires. Your responses in this research will be confidential so there will be no way of linking your responses with your identity. Data from this study will be held in a locked lab room for up to ten years and on a password protected computer to which only investigators and associated personnel will have access. Should the data be used for any secondary analyses, you will be made aware. After this period, the data will be destroyed.

INCENTIVES TO PARTICIPATE: You will receive $\$ 12$ for your participation in this study.

VOLUNTARY NATURE OF PARTICIPATION: Participation is voluntary. Your choice of whether to participate has no bearing on your past, present, or future relationship with Ryerson University. If you participate, you are free to withdraw your consent and stop participation at any time without penalty or loss of benefits to which you are allowed. Should you withdraw from the study, you will still receive your token of appreciation.

QUESTIONS: If you have any questions, please ask now otherwise you may contact: Sara Gallant (ㄱallan@psych.ryerson.ca) or Dr. Lixia Yang (lixiay@psych.ryerson.ca). If you have questions regarding your rights as a participant, you may contact the Ryerson University Research Ethics Board c/o Office of the Vice President, Research and Innovation, Ryerson University 350 Victoria Street Toronto, ON M5B 2K3, 416-979-5042.

AGREEMENT: Your signature indicates that you have read this form and have had a chance to ask any questions you have. It also indicates that you agree to participate and understand that you can withdraw at any point. You have been given a copy of this agreement. By signing, you are aware that you are not giving up any of your legal rights.

$\overline{\text { Name of Participant (please print) }} \overline{\text { Signature of Participant }} \quad \overline{\text { Date }}$

$\overline{\text { Signature of Investigator }} \quad \overline{\text { Date }}$




\title{
Appendix V: Consent Form for Young Adults in Experiment 2
}

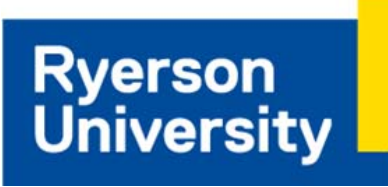 \\ Consent Agreement
}

Ryerson University, Department of Psychology

Please read this consent form so that you understand what your participation involves. Before you consent, please ask any questions you may have with regards to your participation.

\section{STUDY TITLE: The influence of value on memory}

INVESTIGATORS: This research study is being conducted by Sara Gallant ( $\mathrm{PhD}$ candidate) and Dr. Lixia Yang (Associate Professor) from the Department of Psychology at Ryerson University.

PURPOSE OF THE STUDY: The purpose of this cognitive psychology study is to assess how we perceive and remember information that varies in high- vs. low-value. Twenty-four older and 24 young adults will be invited to participate in this study.

DESCRIPTION OF THE STUDY: If you decide to participate, your visit will last approximately 60 minutes. After signing the consent form, you will be asked to complete a task during which you will study a series of different types of words. Some of these words will be designated as high-value items ( +10 points) while others will be low-value items (-10 points). Your goal is to remember as many high-value items while suppressing the low-value items. Following the study phase, a recognition task will be administered in which you will be asked to indicate whether you previously studied the word or if it is new. This design allows us to determine your ability to prioritize high-value information in attention and memory while deprioritizing low-value information. After the computer task, you will be asked to complete questionnaires on your mood, anxiety level, demographic and health information (e.g., age, gender, education level, medication use, health condition). At the end of the session, you will receive your incentive, regardless of whether all aspects of the study have been completed.

WHAT IS EXPERIMENTAL IN THIS STUDY: From a technical or procedural point of view, part of this study is considered "experimental" because the procedure allows us to examine the impact of one variable (the "independent variable"; e.g., aging) on another variable (the "dependent variable"; e.g., memory). More information about the independent and dependent variables will be provided at the end of the session.

RISKS: This is a minimal risk study. However, minor risks may include discomfort from the questions being asked due to their personal nature. Participants may choose to refuse participation in any aspect of the research (e.g., responding to questionnaire items). Any discomfort is expected to be temporary and not greater than what you might experience in a typical day. If any aspect of this study makes you uncomfortable, you may temporarily or permanently discontinue participation without penalty and you will still be fully compensated. 
BENEFITS: We hope the study will benefit society and science by providing a greater understanding of how certain mental abilities change as we age. Participants will receive no direct benefit although information gained from the study may improve their knowledge on the topic of cognition and aging. When the session is over, we will describe the purpose and hypotheses of the study to you in more detail.

CONFIDENTIALITY: You will not put your name on the questionnaires. You will be asked to sign only this consent form, and both the consent form and the background questionnaire will be filed separately from your other questionnaires. Your responses in this research will be confidential so there will be no way of linking your responses with your identity. Data from this study will be held in a locked lab room for up to ten years and on a password protected computer to which only investigators and associated personnel will have access. Should the data be used for any secondary analyses, you will be made aware. After this period, the data will be destroyed.

INCENTIVES TO PARTICIPATE: You will receive one participation credit to use towards your PSY102/202 course. If you prefer to 'walk through' the study (i.e., to observe the research but not provide any personal data), you will still be given the one credit assuming you have not already received the maximum allotted for research participation. Please be advised that a 'walk through' is not a short version of the study, but rather takes you through the full session.

VOLUNTARY NATURE OF PARTICIPATION: Participation is voluntary. Your choice of whether to participate has no bearing on your past, present, or future relationship with Ryerson University. If you participate, you are free to withdraw your consent and stop participation at any time without penalty or loss of benefits to which you are allowed. Should you withdraw from the study, you will still receive your token of appreciation.

QUESTIONS: If you have any questions, please ask now otherwise you may contact: Sara Gallant (s7gallan@psych.ryerson.ca) or Dr. Lixia Yang (lixiay@psych.ryerson.ca). If you have any questions regarding your involvement in the SONA participant pool, please contact thepool@psych.ryerson.ca. If you have questions regarding your rights as a participant, contact the Ryerson University Research Ethics Board c/o Office of the Vice President, Research and Innovation, Ryerson University 350 Victoria Street Toronto, ON M5B 2K3, 416-979-5042.

AGREEMENT: Your signature indicates that you have read this form and have had a chance to ask any questions you have. It also indicates that you agree to participate and understand that you can withdraw at any point. You have been given a copy of this agreement. By signing, you are aware that you are not giving up any of your legal rights.

\footnotetext{
$\overline{\text { Name of Participant (please print) }} \overline{\text { Signature of Participant }} \quad$ Date
}

$\overline{\text { Signature of Investigator }} \quad \overline{\text { Date }}$ 


\section{Appendix VI: Debriefing Form for Experiment 2}

\section{Ryerson University}

Debriefing: Aging and the influence of value on memory

This study examined the influence of value on young and older adults' memory for information that varied in emotional tone (i.e., positive, negative, or neutral words). Examining how these processes are influenced by emotion is important to consider as research has shown age differences in emotional processing, in which older adults show a bias toward positive and away from negative information whereas young adults show a bias toward negative information (the age-related positivity effect; Mather \& Carstensen, 2005). In a prior study, we found that young and older adults were better at remembering words cued as to-be-remembered (TBR) relative to those cued as to-be-forgotten (TBF) regardless of the information's emotional tone (Gallant \& Yang, 2014). We also found that older adults were better at remembering the source (i.e., whether it was TBR or TBF) of the word particularly if it was positive and TBR but not when words were positive and TBF. In contrast, young adults' source memory for TBR and TBF words was not influenced by emotion. This may suggest that older adults have a preference to remember high-priority positive information, consistent with the age-related positivity effect.

In the current study, we are attempting to replicate and extend this effect by using numeric point value $(-10,+10)$ cues to signal the importance of words instead of conceptual cues to remember or forget. To determine how this is influenced by emotion, we manipulated the emotional tone of words (positive, negative, or neutral) in addition to the value $(+10,-10)$ of information (the independent variables) during the study phase. After this, we measured your memory (the dependent variable) for all words regardless of their prior value to determine whether high-value words were prioritized in memory and low-value words were suppressed. If the emotional effects observed in older adults' source memory for TBR and TBF positive words in our prior study were due to prioritization of high-value positive words in memory, then we should replicate our previous pattern of findings using +10 and -10 point-values (i.e., better source memory for +10 vs. -10 positive items in older adults).

For more information regarding this study, you may consult the following sources:

Gallant, S. N., \& Yang, L. (2014). Positivity effect in source attributions of emotional and nonemotional words during item-based directed forgetting. Frontiers in Psychology: Cognition, 5, article: 1334.

Mather, M. \& Carstensen, L. L. (2005). Aging and motivated cognition: The positivity effect in attention and memory. Trends in Cognitive Sciences, 9(10), 496-502.

Please do not hesitate to ask the experimenter questions. Feel free to contact the principal investigator, Sara Gallant (그gallan@psych.ryerson.ca) or Dr. Lixia Yang (lixiay@ryerson.ca) if you have any questions regarding this project. 


\section{Appendix VII: Consent Form for Older Adults in Experiment 3}

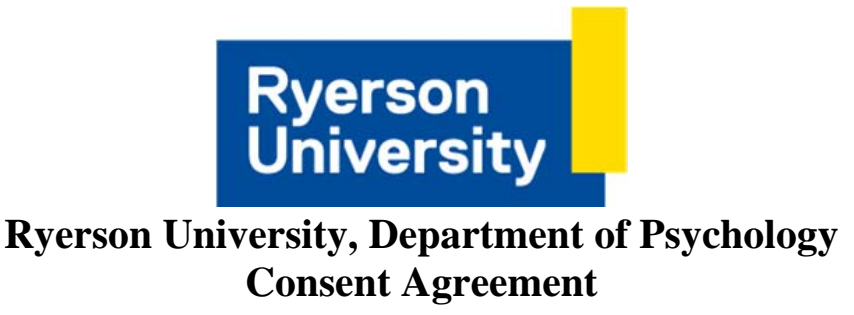

You are being invited to participate in a study. Please read this consent form and ask the experimenter any required questions so that you understand what your participation involves.

\section{STUDY TITLE: Brain activity underlying cognitive control of memory: Effects of aging and emotion}

INVESTIGATORS: This research study is being conducted by Sara Gallant ( $\mathrm{PhD}$ candidate) and Dr. Lixia Yang (Associate Professor) from the Department of Psychology at Ryerson University.

PURPOSE OF THE STUDY: The purpose of this cognitive psychology study is to assess how aging and emotion affects brain activity during completion of a cognitive control task. Twentyeight older and 28 young adults will be invited to participate in this study.

DESCRIPTION OF THE STUDY: If you decide to participate, your visit will last approximately two hours. First, you will read and sign a consent form so that you are aware of all experimental procedures as well as an information sheet regarding how electroencephalography (EEG) is recorded. Following this, you will be asked to complete a learning task during which you will study a series of different types of words that you will need to either remember or forget for a later recognition memory task. While completing this task, your electrical brain activity will be recorded from an EEG cap that will be fit to your head. During the recognition task, you will be shown the words you learned as well as several new words and will identify which items you learned and which are new. Following the computer tasks, you will be asked to complete questionnaires that will assess your mood and anxiety level. Some basic demographic and health information will also be collected in a background questionnaire (e.g., age, gender, education level, medication use, health condition). At the end of the session, you will receive your incentive payment, regardless of whether all aspects of the experiment have been completed.

WHAT IS EXPERIMENTAL IN THIS STUDY: From a technical or procedural point of view, part of this study is considered "experimental" because the procedure allows us to examine the impact of one variable (the "independent variable"; e.g., aging) on another variable (the "dependent variable"; e.g., memory). More information about the independent and dependent variables will be provided at the end of the session.

RISKS: This is a minimal risk study. There are no known long-term risks associated with EEG recording, although you may experience some discomfort from wearing the electrode cap. If you have temporal-mandibular joint (TMJ) disease or any recurrent problems with your head or neck, then you should not take part in this study. Fatigue is also a possibility because of the length of the task (although short breaks will be provided). Should you wish, you may 
discontinue participation either temporarily or permanently, at any point during the experiment and still receive your incentive payment.

BENEFITS: We hope the study will benefit society and science by providing a greater understanding of how certain mental abilities map onto brain activity and change as we age. Participants will receive no direct benefit although information gained from the study may improve knowledge surrounding the topic of cognition and aging. When the session is over, we will describe the purpose and hypotheses of the study to you in more detail.

CONFIDENTIALITY: You will not put your name on the questionnaires. You will be asked to sign only this consent form, and both the consent form and the background questionnaire will be filed separately from your other questionnaires. Your responses in this research will be confidential so there will be no way of linking your responses with your identity. Data from this study will be held in a locked lab room for up to ten years and on a password protected computer to which only investigators and associated personnel will have access. Should the data be used for any secondary analyses, you will be made aware. After this period, the data will be destroyed.

INCENTIVES TO PARTICIPATE: You will receive \$20 for your participation in this study.

VOLUNTARY NATURE OF PARTICIPATION: Participation is voluntary. Your choice of whether to participate has no bearing on your past, present, or future relationship with Ryerson University. If you participate, you are free to withdraw your consent and stop participation at any time without penalty or loss of benefits to which you are allowed. Should you withdraw from the study, you will still receive your token of appreciation.

QUESTIONS: If you have any questions, please ask now otherwise you may contact: Sara Gallant (s7gallan@psych.ryerson.ca) or Dr. Lixia Yang (lixiay@psych.ryerson.ca). If you have questions regarding your rights as a participant, contact the Ryerson University Research Ethics Board c/o Office of the Vice President, Research and Innovation, Ryerson University 350 Victoria Street Toronto, ON M5B 2K3, 416-979-5042.

AGREEMENT: Your signature indicates that you have read this form and have had a chance to ask any questions you have. It also indicates that you agree to participate and understand that you can withdraw at any point. You have been given a copy of this agreement. By signing, you are aware that you are not giving up any of your legal rights.

$\overline{\text { Name of Participant (please print) }} \quad \overline{\text { Signature of Participant }} \quad \overline{\text { Date }}$

$\overline{\text { Signature of Investigator }} \overline{\text { Date }}$ 


\title{
Appendix VIII: Consent Form for Young Adults in Experiment 3
}

\author{
Ryerson \\ University \\ Ryerson University, Department of Psychology \\ Consent Agreement
}

You are being invited to participate in a study. Please read this consent form and ask the experimenter any required questions so that you understand what your participation involves.

\section{STUDY TITLE: Brain activity underlying cognitive control of memory: Effects of aging} and emotion

INVESTIGATORS: This research study is being conducted by Sara Gallant ( $\mathrm{PhD}$ candidate) and Dr. Lixia Yang (Associate Professor) from the Department of Psychology at Ryerson.

PURPOSE OF THE STUDY: The purpose of this cognitive psychology study is to assess how aging and emotion affects brain activity during completion of a cognitive control task. Twentyeight older and 28 young adults will be invited to participate in this study.

DESCRIPTION OF THE STUDY: If you decide to participate, your visit will last approximately two hours. First, you will read and sign a consent form so that you are aware of all experimental procedures as well as an information sheet regarding how electroencephalography (EEG) is recorded. Following this, you will be asked to complete a learning task during which you will study a series of different types of words that you will need to either remember or forget for a later recognition memory task. While completing this task, your electrical brain activity will be recorded from an EEG cap that will be fit to your head. During the recognition task, you will be shown the words you learned as well as several new words and will identify which items you learned and which are new. Following the computer tasks, you will be asked to complete questionnaires that will assess your mood and anxiety level. Some basic demographic and health information will also be collected in a background questionnaire (e.g., age, gender, education level, medication use, health condition). At the end of the session, you will receive your incentive payment, regardless of whether all aspects of the experiment have been completed.

WHAT IS EXPERIMENTAL IN THIS STUDY: From a technical or procedural point of view, part of this study is considered "experimental" because the procedure allows us to examine the impact of one variable (the "independent variable"; e.g., aging) on another variable (the "dependent variable"; e.g., memory). More information about the independent and dependent variables will be provided at the end of the session.

RISKS: This is a minimal risk study. There are no known long-term risks associated with EEG recording, although you may experience some discomfort from wearing the electrode cap. If you have temporal-mandibular joint (TMJ) disease or any recurrent problems with your head or neck, then you should not take part in this study. Fatigue is also a possibility because of the length of the task (although short breaks will be provided). Should you wish, you may 
discontinue participation either temporarily or permanently, at any point during the experiment and still receive your incentive payment.

BENEFITS: We hope the study will benefit society and science by providing a greater understanding of how certain mental abilities map onto brain activity and change as we age. Participants will receive no direct benefit although information gained from the study may improve knowledge surrounding the topic of cognition and aging. When the session is over, we will describe the purpose and hypotheses of the study to you in more detail.

CONFIDENTIALITY: You will not put your name on the questionnaires. You will be asked to sign only this consent form, and both the consent form and the background questionnaire will be filed separately from your other questionnaires. Your responses in this research will be confidential so there will be no way of linking your responses with your identity. Data from this study will be held in a locked lab room for up to ten years and on a password protected computer to which only investigators and associated personnel will have access. Should the data be used for any secondary analyses, you will be made aware. After this period, the data will be destroyed.

INCENTIVES TO PARTICIPATE: You will receive two credits towards your PSY102/202 course at Ryerson. If you prefer to 'walk through' the study (i.e., to observe the research process but not provide data), you will still receive credits assuming you have not already received the maximum (currently 3\%). Please note a 'walk through' is not a short version of the study, but takes you through the full session, without you providing your individual data.

VOLUNTARY NATURE OF PARTICIPATION: Your choice of whether to participate has no bearing on your past, present, or future relationship with Ryerson University. If you participate, you are free to stop participation at any time without penalty or loss of benefits to which you are allowed. Should you withdraw, you will still receive your token of appreciation.

QUESTIONS: If you have any questions, please ask now otherwise you may contact: Sara Gallant (s7gallan@psych.ryerson.ca) or Dr. Lixia Yang (lixiay@psych.ryerson.ca). If you have any questions regarding your involvement in the SONA participant pool, please contact thepool@psych.ryerson.ca. If you have questions regarding your rights as a participant, contact the Ryerson University Research Ethics Board, c/o Office of the Vice President, Research and Innovation, Ryerson University 350 Victoria Street Toronto, ON M5B 2K3, 416-979-5042.

AGREEMENT: Your signature indicates that you have read this form and have had a chance to ask any questions you have. It also indicates that you agree to participate and understand that you can withdraw at any point. You have been given a copy of this agreement. By signing, you are aware that you are not giving up any of your legal rights.

$\overline{\text { Name of Participant (please print) }} \overline{\text { Signature of Participant }} \quad \overline{\text { Date }}$

$\overline{\text { Signature of Investigator }} \quad \overline{\text { Date }}$ 


\section{Appendix IX: Debriefing Form for Experiment 3}

\section{Ryerson University}

Debriefing: Brain activity underlying intentional forgetting: Effects of aging and emotion

This study is examining the effects of age and emotion (independent variables) on brain activity associated with cognitive control of memory (dependent variables). Cognitive control was measured by asking you to remember certain relevant ('to-be-remembered'; TBR) words while intentionally forgetting irrelevant ('to-be-forgotten'; TBF) words for a later memory test. This was done while electrical brain activity was measured with electroencephalography (EEG) to determine the representation of this behaviour in the brain. We also looked at how the emotional tone of words (i.e., positive, negative, or neutral) would impact brain activity across young and older adults. The effects of emotion are particularly important to consider as research has shown age differences in emotional information processing, such that older adults show a bias toward positive and away from negative information whereas young adults show a bias toward negative information (Reed \& Carstensen, 2012). Age differences in emotional information processing have also been evident in the brain, with reduced activity toward negative stimuli in older adults (Kisley et al., 2007). Whether similar effects will be observed for measures of cognitive control of memory, however, is unknown.

In this study, we expect to find greater activity in certain areas of the brain, depending on the type of word being processed (i.e., a TBR or TBF item) as well as the emotional tone of the word (i.e., positive, negative, or neutral). These patterns may also differ across age groups as older adults have previously shown different brain activity patterns relative to young adults when processing positive vs. negative stimuli and when engaging in complex cognitive tasks.

We hope this study will benefit both society and science by informing us about how various mental functions change due to age and how these processes are represented in the brain.

For more information, you can consult the following sources:

Gallant, S. N., \& Yang, L. (2015). Directed forgetting. In S. K. Whitbourne (Ed.), The Encyclopedia of Adulthood and Aging. Wiley-Blackwell.

Kisley, M. A., Wood, S., \& Burrows, C. L. (2007). Looking at the sunny side of life: Age-related change in event-related potential measure of the negativity bias. Psychological Science, 18, 838-843. doi: $10.1111 / \mathrm{j} .1467-9280.2007 .01988 . x$

Reed, A. E., \& Carstensen, L. L. (2012). The theory behind the age-related positivity effect. Frontiers in Psychology, 3, 1-9. doi: 10.3389/fpsyg.2012.00339.

Please do not hesitate to ask questions. If you have questions later, contact Sara Gallant (7gallan@psych.ryerson.ca) or Dr. Lixia Yang (ixiay@ryerson.ca). If you have questions about your rights as a participant contact the Ryerson Research Ethics Board at 416-979- 5000 ext. 4791 or rebchair@ryerson.ca. 
Appendix X: Stimuli selected for Experiments 1-3 from the Affective Norms for English Words database (Bradley \& Yang, 1999)

\begin{tabular}{|c|c|c|}
\hline Negative & Positive & Neutral \\
\hline alone & angel & alley \\
\hline blind & bath & aloof \\
\hline blister & beauty & appliance \\
\hline cemetery & bed & black \\
\hline coward & bird & blasé \\
\hline cut & bless & board \\
\hline dead & breeze & cannon \\
\hline death & brother & coarse \\
\hline discomfort & cake & contents \\
\hline dummy & carefree & context \\
\hline failure & comfort & corner \\
\hline fat & dream & corridor \\
\hline fault & elegant & custom \\
\hline feeble & gentle & dark \\
\hline fever & grateful & errand \\
\hline gloom & heal & excuse \\
\hline grief & hug & gender \\
\hline handicap & leisurely & habit \\
\hline ignorance & luxury & haphazard \\
\hline illness & melody & knot \\
\hline immature & music & lump \\
\hline impair & nature & material \\
\hline inferior & ocean & medicine \\
\hline lost & pillow & muddy \\
\hline messy & politeness & obey \\
\hline moody & protected & odd \\
\hline neglect & rainbow & passage \\
\hline obesity & respectful & patient \\
\hline poverty & reward & privacy \\
\hline rat & safe & quart \\
\hline sad & satisfied & rock \\
\hline scar & secure & sheltered \\
\hline sick & sky & shy \\
\hline slum & snuggle & spray \\
\hline stink & soft & stagnant \\
\hline stupid & sunset & stiff \\
\hline trash & twilight & stomach \\
\hline unhappy & useful & tower \\
\hline useless & warmth & trumpet \\
\hline waste & wise & writer \\
\hline
\end{tabular}




\section{Appendix XI: MATLAB script used for signal processing and ICA}

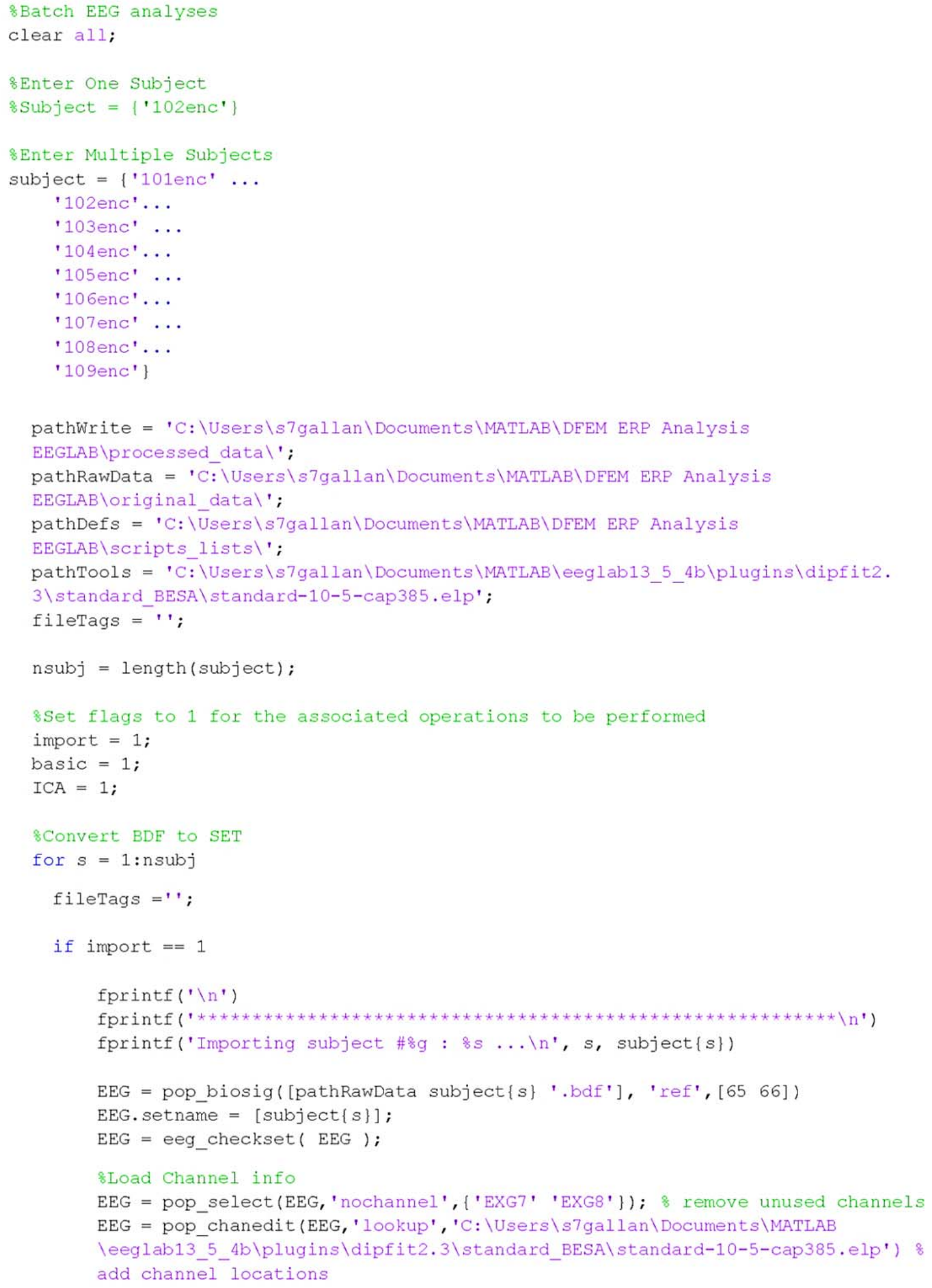




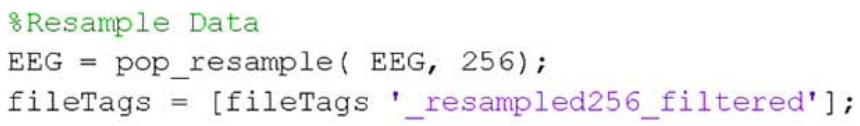

end

B Basic processing

if basic $==1$

fileTags $=[$ fileTags ' BIN'];

fprintf('\n')

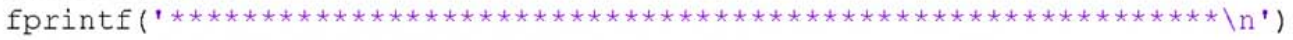

fprintf('BASIC PROCESSSING FOR subject \#sg : $8 s \ldots \backslash n ', s$, subject $\{s\}$ )

$8 \mathrm{EEG}=$ pop_loadset ( 'filename', [subject $\{s\}$ '_resampled256.set'], 'filepath', pathwrite);

EEG $=$ pop reref $\left(\operatorname{EEG},\left[\begin{array}{ll}65 & 66\end{array}\right]\right) ;$

$\mathrm{EEG}=$ pop_creabasiceventlist (EEG, 'Eventlist', 'delme.txt', 'Boundarystring', \{ 'boundary' \}, 'BoundaryNumeric', (-99 \});

diary ('DEEM enc AssignedBins.txt');

$\operatorname{disp}(\operatorname{subject}\{s\})$;

$\mathrm{EEG}=$ pop_binlister( EEG, 'BDE', [pathDefs 'DFEM_enc_binList.txt'], 'ImportEL' ,' 'no', 'Saveas', 'off', 'SendeL2', 'EEG\&Text', 'Warning', 'ōn'); sassign events (i.e. codes) into bins (i.e., experimental conditions)

diary off;

8Highpass filter at $0.1 \mathrm{~Hz}$

$\mathrm{EEG}=$ pop_basicfilter( EEG, 1:68, 'Cutoff', 0.1, 'Design', 'butter', 'Eilter', 'highpass', 'Order', 2, 'RemoveDC', 'on' );

s.Low-Pass filter at $30 \mathrm{~Hz}$

$\mathrm{EEG}=$ pop_basicfilter( EEG, 1:68, 'Cutoff', 30, 'Design', 'butter', 'Filter', 'lowpass', 'Order', 4, 'RemoveDC', 'on' );

$\mathrm{EEG}=$ pop epochbin( EEG , [-200 1200], 'pre'); sextract epochs (and get rid of rest of file)

\$EEG = pop saveset( EEG, 'filename', [subject $\{s\}$ fileTags '.set'], 'filepath', pathWrite);

end

$\mathrm{EEG}=$ eeg_checkset $(\mathrm{EEG})$;

sICA analysis

if $\mathrm{ICA}==1$

fileTags $=[$ fileTags '_ICA'];

$\mathrm{EEG}=$ pop runica (EEG, 'extended',1,'logfile', [EEG. setname, '-ICAlog.txt']);

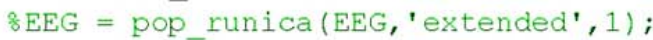

EEG = pop_saveset(EEG,'filename', [subject\{s\} fileTags '.set'], 'filepath',

pathWrite);

end

end 


\section{References}

American Psychiatric Association (2013). Diagnostic and statistical manual of mental disorders (5th ed.). Arlington, VA: American Psychiatric Publishing

Anderson, M. C., \& Green, C. (2001). Suppressing unwanted memories by executive control. Nature, 410, 366-369. doi: 10.1038/35066572

Anderson, M. C. (2005). The role of inhibitory control in forgetting unwanted memories: A consideration of three methods. In C. M. MacLeod \& B. Uttl (Eds.) Dynamic cognitive processes (pp. 159-190). Tokyo: Springer-Verlag.

Anderson, M. C., \& Levy, B.J. (2009). Suppressing unwanted memories. Current Directions in Psychological Science, 18, 184-194. doi: 10.1111/j.1467-8721.2009.01634.x

Anderson, M. C., \& Hanslmayr, S. (2014). Neural mechanisms of motivated forgetting. Trends in Cognitive Sciences, 18, 279-282. doi: 10.1016/j.tics.2014.03.002

Anderson, N. D., Ebert, P. L., Jennings, J. M., Grady, C. L., Cabeza, R., \& Graham, S. J. (2008). Recollection- and familiarity-based memory in healthy aging and amnestic mild cognitive impairment. Neuropsychology, 22, 177-187. doi:10.1037/08944105.22.2.177

Andres, P., Van der Linden, M. V., \& Parmentier, F. B. R. (2004). Directed forgetting in working memory: Age-related differences. Memory, 12, 248-256. doi: $10.1080 / 09658210244000612$

Atkinson, R. C., \& Shiffrin, R. M. (1968). Human memory: A proposed system and its control processes. Psychology of Learning and Motivation, 2, 89-195. doi: 10.1016/S00797421(08)60422-3 
Baddeley, A. D., \& Hitch, G. (1974). Working memory. Psychology of Learning and Motivation, 8, 47-89. doi: 10.1016/S0079-7421(08)60452-1

Bailey, H., Dunlosky, J., \& Hertzog, C. (2010). Metacognitive training at home: Does it improve older adults' learning? Gerontology, 56, 414-420. doi: 10.1159/000266030

Bailey, K., \& Chapman, P. (2012). When can we choose to forget? An ERP study into itemmethod directed forgetting of emotional words. Brain and Cognition, 78, 133-147. doi: 10.1016/j.bandc.2011.11.004

Basden, B. H., Basden, D. R., \& Gargano, G. J. (1993). Directed forgetting in implicit and explicit memory tests: A comparison of methods. Journal of Experimental Psychology: Learning, Memory, and Cognition, 19, 603-616. doi: 10.1037/0278-7393.19.3.603

Basden, B. H., \& Basden, D. R. (1996). Directed forgetting: Further comparisons of the item and list methods. Memory, 4, 633-653. doi: 10.1080/741941000

Basden, B. H., \& Basden, D. R. (1998). Directed forgetting: A contrast of methods and interpretations. In J. M. Golding \& C. M. MacLeod (Eds.), Intentional forgetting: Interdisciplinary approaches (pp. 139-172). Mahwah, NJ: Erlbaum.

Bastin, C., Feyers, D., Majerus, S., Balteau, E., Degueldre, C.,...Collette, F. (2012). The neural substrates of memory suppression: A fMRI exploration of directed forgetting. PLoS ONE, 7, e29905. doi: 10.1371/journal.pone.0029905

Baumeister, R. F., Bratslavsky, E., Finkenauer, C., \& Vohs, K. D. (2001). Bad is stronger than good. Review of General Psychology, 5, 323-370. doi: 10.1037/1089-2680.5.4.323

Bayen, U. J., Murnane, K., \& Erdfelder, E. (1996). Source discrimination, item detection, and multinomial models of source monitoring. Journal of Experimental Psychology: Learning, Memory, and Cognition, 22, 197-215. doi: 10.1037/0278-7393.22.1.197 
Bayen, U. J., Nakamura, G. V., Dupuis, S. E., Yang, C.-L. (2000). The use of schematic knowledge about sources in source monitoring. Memory \& Cognition, 3, 480-500. doi: 10.3758/BF03198562

Beck, D. M., \& Kastner, S. (2009). Top-down and bottom-up mechanisms in biasing competition in the human brain. Vision Research, 49, 1154-1165. doi: 10.1016/j.visres.2008.07.012

Bennion, K. A., Payne, J. D., \& Kensinger, E. A. (2016). The impact of napping on memory for future-relevant stimuli: Prioritization among multiple salience cues. Behavioral Neuroscience, 130, 281-289. doi: 10.1037/bne0000142

Berger, N., Crossman, M., \& Brandt, K. R. (2016). No evidence for age-related differences in item-method directed forgetting of emotional words. The Quarterly Journal of Experimental Psychology, 1-10. doi: 10.1080/17470218.2016.1264433

Bjork, R. A., LaBerge, D., \& Legrand, R. (1968). The modification of short-term memory through instructions to forget. Psychonomic Science, 10, 55-56. doi: 10.3758/BF03331404

Bjork, R. A. (1970). Positive forgetting: The noninterference of items intentionally forgotten. Journal of Verbal Learning and Verbal Behavior, 9, 255-268. doi: 10.1016/S00225371(70)80059-7

Bjork, R. A. (1972). Theoretical implications of directed forgetting. In A. W. Melton \& E. Martin (Eds.), Coding processes in human memory (pp. 217-235). Washington, DC: Winston.

Bjork, R. A. (1998). Intentional forgetting in perspective: Comments, conjectures, and some remembering. In J. M. Golding \& C. M. MacLeod (Eds.), Intentional forgetting: interdisciplinary approaches (pp. 453-481). Mahwah, NJ: Lawrence Erlbaum Associates. 
Bonnesen, J. L., \& Burgess, E. O. (2004). Senior moments: The acceptability of an ageist phrase. Journal of Aging Studies, 18, 123-142. doi: 10.1016/j.jaging.2004.01.008

Bradley, M. M., \& Lang, P. J. (1999). Affective norms for English words (ANEW): Instruction manual and affective ratings. Technical Report C-1, The Center for Research in Psychophysiology, University of Florida. Retrieved from: http://www.uvm.edu/ pdodds/research/papers/others/1999/bradley1999a.pdf

Brandt, K. R., Nielson, M. K., \& Holmes, A. (2013). Forgetting emotional and neutral words: An ERP study. Brain Research, 1501, 21-31. doi: 10.1016/j.brainres.2013.01.019

Brown, S. B. R., van Steenbergen, H., Band, G. P. H., de Rover, M., \& Nieuwenhuis, S. (2012). Functional significance of the emotion-related late positive potential. Frontiers in Human Neuroscience, 6, 1-12. doi: 10.3389/fnhum.2012.00033

Cabeza, R., \& Dennis, N. A. (2012). Frontal lobes and aging: Deterioration and compensation. In D.T. Stuss \& R.T. Knight (Eds), Principles of frontal lobe function ( $2^{\text {nd }}$ ed., pp. 628-652). New York, NY: Oxford University Press.

Carstensen, L. L. (1995). Evidence for a life-span theory of socioemotional selectivity. Psychological Science, 4, 151-156. Retrieved from: http://www.jstor.org/stable/20182356

Carstensen, L. L., \& Mikels, J. A. (2005). At the intersection of emotion and cognition aging and the positivity effect. Current Directions in Psychological Science, 14, 117-121. doi: $10.1111 / \mathrm{j} .0963-7214.2005 .00348 . \mathrm{x}$

Carstensen, L. L., Mikels, J. A., \& Mather, M. (2006). Aging and the intersection of cognition, motivation and emotion. In J.E. Birren, K.W. Schaie (Eds.), Handbook of the psychology of aging (6 ${ }^{\text {th }}$ ed., pp. 343-362). San Diego: Academic Press. 
Carstensen, L. L., Turan, B., Sheibe, S., Ram, N., Ersner-Hershfield, H., Samanez-Larkin, G. R., ...Nesselroade, J. R. (2011). Emotional experience improves with age: Evidence based on over 10 years of experience sampling. Psychology and Aging, 26, 21-33. doi: $10.1037 / \mathrm{a} 0021285$

Castel, A. D., Benjamin, A. S., Craik, F. I., \& Watkins, M. J. (2002). The effects of aging on selectivity and control in short-term recall. Memory \& Cognition, 30, 1078-1085. doi: 10.3758/BF03194325

Castel, A. D. (2007). Aging and memory for numerical information: The role of specificity and expertise in associative memory. Journal of Gerontology: Psychological Sciences, 62, 194-196. doi: 10.1093/geronb/62.3.P194

Castel, A. D., Farb, N., \& Craik, F. I. M. (2007). Memory for general and specific value information in younger and older adults: Measuring the limits of strategic control. Memory \& Cognition, 35, 689-700. doi: 10.3758/BF03193307

Charles, S. T., Mather, M., \& Carstensen, L. L. (2003). Aging and emotional memory: The forgettable nature of negative images for older adults. Journal of Experimental Psychology: General, 132, 310-324. doi: 10.1037/0096-3445.132.2.310

Charles, S. T., \& Carstensen, L. L. (2009). Social and emotional aging. Annual Review of Psychology, 61, 383-409. doi: 10.1146/annurev.psych.093008.100448

Codispoti, M., Ferrari, V., \& Bradley, M. M. (2007). Repetition and event-related potentials: Distinguishing early and late processes in affective picture perception. Journal of Cognitive Neuroscience, 19, 577-586. doi: 10.1162/jocn.2007.19.4.577 
Collette, F., Germain, S., Hogge, M., \& Van der Linden, M. (2009). Inhibitory control of memory in normal ageing: Dissociation between impaired intentional and preserved unintentional processes. Memory, 17, 104-122. doi: 10.1080/09658210802574146

Collette, F., Grandjean, J., Lorant, C., \& Bastin, C. (2014). The role of memory traces quality in directed forgetting: A comparison of young and older participants. Psychologica Belgica, 54, 310-327. doi: 10.5334/pb.au

Craik, F. I. M., \& Byrd, M. (1982). Aging and cognitive deficits: The role of attentional resources. In F. I. M. Craik \& S. Trehub (Eds.), Aging and cognitive processes (pp. 191211). New York, NY: Plenum.

Dalgleish, T., Taghavi, R., Neshat-Doost, H., Moradi, A., Canterbury, R., \& Yule, W. (2003). Patters of processing bias for emotional information across clinical disorders: A comparison of attention, memory, and prospective cognition in children and adolescents with depression, generalized anxiety, and posttraumatic stress disorder. Journal of Clinical Child and Adolescent Psychology, 32, 10-12. doi: 10.1207/15374420360533022

Davis, S. W., Dennis, N. A., Fleck, M. S., Daselaar, S. M., Cabeza, R. (2008). Que PASA?: The posterior-anterior shift in aging. Cerebral Cortex, 18, 1201-1209. doi: 10.1093/cercor/bhm155

Dennis, N. A., \& Cabeza, R. (2008). Neuroimaging of healthy cognitive aging. In F. I. M. Craik \& T. A. Salthouse (Eds.), Handbook of aging and cognition ( $3^{\text {rd }}$ Ed., pp. 1-54). Mahwah, NJ: Erlbaum.

Dewherst, S. A., \& Parry, L. A. (2000). Emotionality, distinctiveness, and recollective experience. European Journal of Cognitive Psychology, 12, 541-551. doi: $10.1080 / 095414400750050222$ 
Donaldson, W. (1992). Measuring recognition memory. Journal of Experimental Psychology: General, 121, 275-277. doi: 10.1037/0096-3445.121.3.275

Eich, T. S., \& Castel, A. D. (2016). The cognitive control of emotional versus value-based information in younger and older adults. Psychology and Aging, 31, 503-512. doi: 10.1037/pag0000106

Fabiani, M., \& Friedman, D. (1995). Changes in brain activity patterns in aging: The novelty oddball. Psychophysiology, 32, 579-594. doi: 10.1111/j.1469-8986.1995.tb01234.x

Faul, F., Erdfelder, E., Lang, A. G., \& Buchner, A. (2014). G* Power (Version 3.1. 9.2) [software].

Fawcett, J. M., \& Taylor, T. L. (2008). Forgetting is effortful: Evidence from reaction time probes in an item-method directed forgetting task. Memory \& Cognition, 36, 1168-1181. doi: 10.3758/MC.36.6.1168

Flavell, J. H. (1979). Metacognition and cognitive monitoring: A new area of cognitivedevelopmental inquiry. American Psychologist, 34, 906-911. doi: 10.1037/0003066X.34.10.906

Foley, M. A., Johnson, M. K., \& Raye, C. L. (1983). Age-related changes in confusion between memories for thoughts and memories for speech. Child Development, 54, 51-60. doi: $10.2307 / 1129860$

Friedman, D. (2003). Cognition and aging: A highly selective overview of event-related potential (ERP) data. Journal of Clinical and Experimental Neuropsychology, 25, 702-720. doi: 10.1076/jcen.25.5.702.14578 
Friedman, D. (2012). The components of aging. In S. Kappenman \& S. J. Luck (Eds.), The Oxford handbook of event-related potential components (pp. 1-28). New York, NY: Oxford University Press.

Friedman, D. (2013). The cognitive aging of episodic memory: A view based on the eventrelated brain potential. Frontiers in Behavioral Neuroscience, 7, 111. doi: 10.3389/fnbeh.2013.00111

Friedman, M. C., \& Castel, A. D. (2011). Are we aware of our ability to forget? Metacognitive predictions of directed forgetting. Memory \& Cognition, 39, 1448-1456. doi: $10.3758 / \mathrm{s} 13421-011-0115-\mathrm{y}$

Gallant, S. N., \& Yang, L. (2014). Positivity effect in source attributions of arousal-matched emotional and non-emotional information during item-based directed forgetting. Frontiers in Psychology: Cognition, 5, 1-8. doi: 10.3389/fpsyg.2014.01334

Gallant, S. N., \& Yang, L. (2015). Directed forgetting. In S. K. Whitbourne (Ed.), The encyclopedia of adulthood and aging (pp. 347-349). Sussex, UK: Wiley Blackwell.

Gallant, S. N., \& Dyson, B. J. (2016). Neural modulation of directed forgetting by valence and arousal. Brain Research, 1648, 306-316. doi: 10.1016/j.brainres.2016.08.009

Gamboz, N., \& Russo, R. (2002). Evidence for age-related equivalence in the directed forgetting paradigm. Brain and Cognition, 48, 366-371. doi: 10.1006/brcg.2001.1379

Gardiner, J. M. (1988). Functional-aspects of recollective experience. Memory \& Cognition, 16, 309-313. doi:10.3758/BF03197041

Gardiner, J. M., Gawlik, B., \& Richardson-Klavehn, A. (1994). Maintenance rehearsal affects knowing, not remembering; elaborative rehearsal affects remembering, not knowing. Psychonomic Bulletin \& Review, 1, 107-110. doi: 10.3758/BF03200764. 
Gilewski, M. J., Zelinski, E. M., \& Schaie, K. W. (1990). The memory functioning questionnaire for assessment of memory complaints in adulthood and old age. Psychology and Aging, 5, 482-490. doi: 10.1037/0882-7974.5.4.482

Goernert, P. N., Corenblum, B., \& Otani, H. (2011). Directed forgetting of faces: The role of response criterion. The Quarterly Journal of Experimental Psychology, 64, 1930-1938. doi: $10.1080 / 17470218.2011 .595806$

Goodman, L., \& Kruskal, W. H. (1954). Measures of association for cross classifications. Journal of the American Statistical Association, 49, 732-764. doi:10.2307/2281536

Grady, C. L., Maisog, J. M., Horwitz, B., Ungerleider, L. G., Mentis, M. J., Salerno,...Haxby. J. V. (1994). Age-related changes in cortical blood flow activation during visual processing of faces and location. Journal of Neuroscience, 14, 1450-1462. doi: 10.1111/j.17496632.1996.tb34396.x

Grady, C. L., \& Craik, F. I. M.(2000). Changes in memory processing with age. Current Opinion in Neurobiology, 10, 224-231. doi: 10.1016/S0959-4388(00)00073-8

Grady, C. L. (2012). The cognitive neuroscience of aging. Nature Reviews Neuroscience, 13, 491-505. doi: 10.1038/nrn3256.

Haber, S. (2012). The neurological components of metamemory monitoring: JOL accuracy in younger and older adults (Unpublished doctoral dissertation). Rice University: Houston, TX.

Hajcak, G., \& Olvet, D. M. (2008). The persistence of attention to emotion: Brain potentials during and after picture presentation. Emotion, 8, 250-255. doi: 10.1037/15283542.8 .2 .250 
Hamann, S. (2001). Cognitive and neural mechanisms of emotional memory. Trends in Cognitive Science, 5, 394-400. doi:10.1016/S1364-6613(00)01707-1

Hasher, L., \& Zacks, R. T. (1988). Working memory, comprehension, and aging: A review and a new view. In G. H. Bower (Ed.), The psychology of learning and motivation: Advances in research and theory, vol. 22 (pp. 193-225). San Diego, CA: Academic Press.

Hashtroudi, S., Johnson, M. K., \& Chrosniak, L. D. (1989). Aging and source monitoring. Psychology and Aging, 4, 106-112. doi: 10.1037/0882-7974.4.1.106

Hashtroudi, S., Johnson, M. K., \& Chrosniak, L. D. (1990). Aging and qualitative characteristics of memories for perceived and imagined complex events. Psychology and Aging, 5, 119126. doi: $10.1037 / 0882-7974.5 .1 .119$

Hauswald, A., Schulz, H., Iordanov, T., \& Kissler, J. (2010). ERP dynamics underlying successful directed forgetting of neutral but not negative pictures. Social Cognitive and Affective Neuroscience, 6, 450-459. doi: 10.1093/scan/nsq061

Hayes, A. F. (2012). PROCESS: A versatile computational tool for observed variable mediation, moderation, and conditional process modeling [White paper]. Retrieved from http://www.afhayes.com/public/process2012.pdf.

Hedden, T., \& Gabrieli, J. D. E. (2004). Insights into the ageing mind: A view from cognitive neuroscience. Nature Reviews, 5, 89-96. doi: 10.1038/nrn1323

Hertzog, C., Dixon, R. A., \& Hultsch, D. F. (1990). Relationships between metamemory, memory predictions, and memory task performance in adults. Psychology and Aging, 5, 215-227. doi: 10.1037/0882-7974.5.2.215 
Hertzog C., \& Hultsch, D. F. (2000). Metacognition in adulthood and old age. In F. I. M. Craik (Ed). The handbook of aging and cognition (pp. 417-466). Mahwah, NJ: Lawrence Erlbaum Associates.

Hertzog, C., \& Dunlosky, J. (2011). Metacognition in later adulthood: Spared monitoring can benefit older adults' self-regulation. Current Directions in Psychological Science, 20, 167-173. doi: 10.1177/0963721411409026

Hess, T. M. (2014). Selective engagement of cognitive resources: Motivational influences on older adults' cognitive functioning. Psychological Science, 9, 388-407. doi: $10.1177 / 1745691614527465$

Hogge, M., Adam, S., \& Collette, F. (2008). Directed forgetting and aging: The role of retrieval processes, processing speed, and proactive interference. Aging, Neuropsychology, and Cognition, 15, 471-491. doi: 10.1080/13825580701878065

Isaacowitz, D. M., Wadlinger, H. A., Goren, D., \& Wilson, H. R. (2006). Selective preference in visual fixation away from negative images in old age? A visual eye-tracking study. Psychology and Aging, 21, 40-48. doi: 10.1037/0882-7974.21.1.40

Johnson, M. K., Hashtroudi, S., \& Lindsay, D. S. (1993). Source monitoring. Psychological Bulletin, 114, 3-28. doi: 10.1037/0033-2909.114.1.3

Katzman, R., Brown, T., Fuld, P., Peck, A., Schechter, R., \& Schimmel, H. (1983). Validation of a short orientation-memory concentration test of cognitive impairment. American Journal of Psychiatry, 140, 734-739.

Kensinger, E. A., \& Corkin, S. (2003). Memory enhancement for emotional words: Are emotional words more vividly remembered than neutral words? Memory \& Cognition, 31, 1169-1180. doi: 10.3758/BF03195800 
Kensinger, E. A., \& Corkin, S. (2004). Two routes to emotional memory: Distinct neural processes for valence and arousal. Proceedings of the National Academy of Sciences, 101, 3310-3315. doi: 10.1073/pnas.0306408101

Kensinger, E. A. (2008). Age differences in memory for arousing and nonarousing emotional words. Journal of Gerontology: Psychological Sciences, 63B, 13-18. doi: 10.1093/geronb/63.1.P13

Kisley, M. A., Wood, S., \& Burrows, C. L. (2007). Looking at the sunny side of life: Age-related change in event-related potential measure of the negativity bias. Psychological Science, 18, 838-843. doi: 10.1111/j.1467-9280.2007.01988.x

Knight, M., Seymour, T. L., Gaunt, J. T., Baker, C., Nesmith, K., \& Mather, M. (2007). Aging and goal-directed emotional attention: Distraction reverses emotional biases. Emotion, 7, 705-714. doi: 10.1037/1528-3542.7.4.705

Koen, J. D., \& Yonelinas, A. P. (2016). Recollection, not familiarity, decreases in healthy ageing: Converging evidence from four estimation methods. Memory, 24, 75-88. doi: $10.1080 / 09658211.2014 .985590$

Koriat, A. (1997). Monitoring one's own knowledge during study: A cue-utilization approach to judgments of learning. Journal of Experimental Psychology: General, 126, 349-370. doi: 10.1037/0096-3445.126.4.349

Koriat, A. (2007). Metacognition and consciousness. In P. D. Zelazo, M. Moscovitch, \& E. Thompson (Eds.), The Cambridge handbook of consciousness (pp. 289-325). New York, NY: Cambridge University Press. 
Kuhlmann, B. G., \& Touron, D. (2011). Older adults' use of metacognitive knowledge in source monitoring: Spared monitoring but impaired control. Psychology \& Aging, 26, 143-149. doi: $10.1037 / \mathrm{a} 0021055$

Kuhlmann, B. G., \& Boywitt, C. D. (2015). Aging, source memory, and the experience of “remembering”. Aging, Neuropsychology, and Cognition, 23, 477-498. doi: $10.1080 / 13825585.2015 .1120270$

Kuhlmann, B. G., \& Bayen, U. J. (2016). Metacognitive aspects of source monitoring. In J. Dunlosky \& U. Tauber (Eds.), The oxford handbook of metamemory (pp. 149-168). New York, NY: Oxford University Press.

Labar, K. S., \& Cabeza, R. (2006). Cognitive neuroscience of memory. Nature Reviews Neuroscience, 7, 54-64. doi:10.1038/nrn1825

Labouvie-Vief, G. (2003). Dynamic integration: Affect, cognition, and the self in adulthood. Current Directions in Psychological Science, 12, 201-206. doi: 10.1046/j.09637214.2003.01262.x

Lang, P. J., Greenwald, M. K., Bradley, M. M., \& Hamm, A. O. (1993). Looking at pictures: Affective, facial, visceral, and behavioral reactions. Psychophysiology, 30, 261-273. doi: 10.1111/j.1469-8986.1993.tb03352.x

Liu, Y., Huang, H., McGinnis-Deweese, M., Keil, A., \& Ding, M. (2012). Neural substrate of the late positive potential in emotional processing. Journal of Neuroscience, 32, 14563-72. doi: 10.1523/JNEUROSCI.3109-12.2012.

Lovibond, S. H., \& Lovibond, P. F. (1995). Manual for the Depression Anxiety Stress Scales. Sydney: The Psychology Foundation of Australia. 
Luck, S. J. (2014). An introduction to the event-related potential technique ( $2^{\text {nd }}$ ed.). Cambridge, MA: MIT Press.

Lustig, C., Hasher, L., \& Zacks, R. (2007). Inhibitory deficit theory: Recent developments in a "New View". In D. Gorfein \& C. M. MacLeod (Eds.), Inhibition in cognition (pp. 145-162). Washington, DC: American Psychological Association.

MacLeod, C., \& McLaughlin, K. (1995). Implicit and explicit memory bias in anxiety: A conceptual replication. Behaviour Research and Therapy, 33, 1-14. doi: 10.1016/00057967(94)E0004-3

MacLeod, C. M. (1998). Directed forgetting. In J. M. Golding \& C. M. MacLeod (Eds.), Intentional forgetting: Interdisciplinary approaches (pp. 1-57). Mahwah, NJ: Lawrence Erlbaum Associates.

MacLeod, C. M. (1999). The item and list methods of directed forgetting: Test differences and the role of demand characteristics. Psychonomic Bulletin \& Review, 6, 123-129. doi: 10.3758/BF03210819

Mather, M., Johnson, M. K., \& De Leonardis, D. M. (1999). Stereotype reliance in source monitoring: Age differences and neuropsychological test correlates. Cognitive Neuropsychology, 16, 437-458. doi: 10.1080/026432999380870

Mather, M., \& Carstensen, L. L. (2003). Aging and attentional biases for emotional faces. Psychological Science, 14, 409-415. doi: 10.1111/1467-9280.01455

Mather, M., \& Carstensen, L. L. (2005). Aging and motivated cognition: The positivity effect in attention and memory. Trends in Cognitive Sciences, 9, 496-502. doi: 10.1016/j.tics.2005.08.005 
Mather, M., \& Knight, M. (2005). Goal-directed memory: The role of cognitive control in older adults' emotional memory. Psychology and Aging, 20, 554-570. doi: 10.1037/08827974.20.4.554

Mather, M. (2010). Aging and cognition. Wiley Interdisciplinary Reviews: Cognitive Science, 1, 346-362. doi: $10.1002 /$ wcs. 64

Mather, M. (2012). The emotion paradox in the aging brain. Annals of the New York Academy of Sciences, 1251, 33-49. doi: 10.1111/j.1749-6632.2012.06471.x

May, C. P., Rahhal, T., Berry, E. M., \& Leighton, E. A. (2005). Aging, source memory, and emotion. Psychology and Aging, 20, 571-578. doi: 10.1037/0882-7974.20.4.571

Maylor, E. A. (1993). Aging and forgetting in prospective and retrospective memory tasks. Psychology and Aging, 8, 420-428. doi: 10.1037/0882-7974.8.3.420

Metcalfe, J. \& Dunlosky, J. (2008). Metamemory. In H.L. Roediger, III (Ed.), Learning and memory: A comprehensive reference (pp. 349-362). Oxford: Elsevier.

Migo, E. M., Mayes, A. R., \& Montaldi, D. (2012). Measuring recollection and familiarity: Improving the remember/know procedure. Consciousness and Cognition, 21, 1435-1455. doi: 10.1016/j.concog.2012.04.014

Minnema, M. T., \& Knowlton, B. J. (2008). Directed forgetting of emotional words. Emotion, 8, 643-652. doi: 10.1037/a0013441

Mitchell, K. J., \& Johnson, M. K. (2009). Source monitoring 15 years later: What have we learned from fMRI about the neural mechanisms of source memory? Psychological Bulletin, 135, 638-677. doi: 10.1037/a0015849 
Mitchell, K. J. (2016). The cognitive neuroscience of source monitoring. In J. Dunlosky \& U. Tauber (Eds), The oxford handbook of metamemory (pp. 425-449). New York, NY: Oxford University Press.

Muther, W. S. (1965). Erasure or partitioning of short-term memory. Psychonomic Science, 3, 429-430. doi: 10.3758/BF03343215

Naveh-Benjamin, M. (2000). Adult age differences in memory performance: Tests of associative deficit hypothesis. Journal of Experimental Psychology, 26, 1170-1187. doi: $10.1037 / 0278-7393.26 .5 .1170$

Nelson, T. O., \& Dunlosky, J. (1991). When people's judgments of learning (JOLs) are extremely accurate at predicting subsequent recall: The "delayed-JOL effect". Psychological Science, 2, 267-271. doi: 10.1111/j.1467-9280.1991.tb00147.x

Nelson, T. O., \& Narens, L. (1990). Metamemory: A theoretical framework and new findings. Psychology of Learning and Motivation, 26, 125-173. doi: 10.1016/S00797421(08)60053-5

Nieuwenhuis, S., Aston-Jones, G., \& Cohen, J. D. (2005). Decision making, the P3, and the locus coeruleus--norepinephrine system. Psychological Bulletin, 131, 510-532. doi: $10.1037 / 0033-2909.131 .4 .510$

Nowicka, A., Jednoróg, K., Wypych, M., \& Marchewka, A. (2009). Reversed old/new effect for intentionally forgotten words: An ERP study of directed forgetting. International Journal of Psychophysiology, 71, 97-102. doi: 10.1016/j.ijpsycho.2008.06.009

Nowicka, A., Marchewka, A., Jednorog, K., Tacikowski, P., \& Brechmann, A. (2011). Forgetting of emotional information is hard. An fMRI study of directed forgetting. Cerebral Cortex, 21, 539-549. doi:10.1093/cercor/bhq117 
Ochsner, K. N., \& Gross, J. (2005). The cognitive control of emotion. Trends in Cognitive Sciences, 9, 242-249. doi: 10.1016/j.tics.2005.03.01

O'Connell, R. G., Balsters, J. H., Kilcullen, S. M., Campbell, W., Bokde, A. W., Lai, R., ... \& Robertson, I. H. (2012). A simultaneous ERP/fMRI investigation of the P300 aging effect. Neurobiology of Aging, 33, 2448-2461. doi: 10.1016/j.neurobiolaging.2011.12.021

Olofsson, J. K., Nordin, S., Sequeira, H., \& Polich, J. (2008). Affective picture processing: An integrative review of ERP findings. Biological Psychology, 77, 247-265. doi: 10.1016/j.biopsycho.2007.11.006

Old, S. R., \& Naveh-Benjamin, M. (2008). Differential effects of age on item and associative measures of memory: A meta-analysis. Psychology and Aging, 23, 104-118. doi: $10.1037 / 0882-7974.23 .1 .104$

Otani, H., Libkuman, T. M., Goernert, P. N., Kato, K., Migita, M., Freehager, S. E., \& Landow, M. P. (2011). Emotion, directed forgetting, and source memory. British Journal of Psychology, 103, 343-358. doi: 10.1111/j.2055-8295.2011.02078.x

Paller, K. A. (1990). Recall and stem-completion priming have different electrophysiological correlates and are modified differentially by directed forgetting. Journal of Experimental Psychology: Learning, Memory, and Cognition, 16, 1021. doi: 10.1037//02787393.16.6.1021

Patrick, R. E., Kiang, M., \& Christensen, B. K. (2015). Neurophysiological correlates of emotional directed-forgetting in persons with Schizophrenia: An event-related brain potential study. International Journal of Psychophysiology. doi: 10.1016/j.jpsycho.2015.01.006. 
Paz-Caballero, M. D., \& Menor, J. (1999). ERP correlates of directed forgetting effects in direct and indirect memory tests. European Journal of Cognitive Psychology, 11, 239-260. doi: $10.1080 / 713752308$

Paz-Caballero, M. D., Menor, J., \& Jiménez, J. M. (2004). Predictive validity of event-related potentials (ERPs) in relation to directed forgetting effects. Clinical Neurophysiology, 115, 369-377. doi: 10.1016/j.clinph.2003.09.011

Pessoa, L. (2009). How do emotion and motivation direct executive control? Trends in Cognitive Sciences, 13, 160-166. doi: 10.1016/j.tics.2009.01.006

Pires, L., Leitão, J., Guerrini, C., \& Simões, M. R. (2014). Event-related brain potentials in the study of inhibition: Cognitive control, source localization and age-related modulations. Neuropsychology Review, 24, 461-490. doi: 10.1007/s11065-014-9275-4

Polich, J. (2007). Updating P300: An integrative theory of P3a and P3b. Clinical Neurophysiology, 118, 2128-2148. doi: 10.1016/j.clinph.2007.04.019

Rahhal, T. A., May, C. P., \& Hasher, L. (2002). Truth and character: Sources that older adults can remember. Psychological Science, 13, 101-105. doi: 10.1111/1467-9280.00419

Reed, A. E., \& Carstensen, L. L. (2012). The theory behind the age-related positivity effect. Frontiers in Psychology, 3, 1-9. doi: 10.3389/fpsyg.2012.00339

Reed, A. E., Chan, L., \& Mikels, J. A. (2014). Meta-analysis of the age-related positivity effect: Age differences in preferences for positive over negative information. Psychology and Aging, 29, 1-15. doi: 10.1037/a0035194

Reid, L. M., \& MacLullich, A. M. (2006). Subjective memory complaints and cognitive impairment in older people. Dementia and Geriatric Cognitive Disorders, 22, 471-485. doi: $10.1159 / 000096295$ 
Rhodes, M. G., \& Tauber, S. K. (2011). The influence of delaying judgments of learning on metacognitive accuracy: A meta-analytic review. Psychological Bulletin, 137, 131-148. doi: $10.1037 / \mathrm{a} 0021705$

Rhodes, M. G., Witherby, A. E., Castel, A. D., \& Murayama, K. (2017). Explaining the forgetting bias effect on value judgments: The influence of memory for a past test. Memory \& Cognition, 45, 362-374. doi:10.3758/s13421-016-0674-z

Rizio, A. A., Dennis, N. A. (2013). The neural correlates of cognitive control: Successful remembering and intentional forgetting. Journal of Cognitive Neuroscience, 25, 297-312. doi: 10.1162/jocn_a_00310

Rizio, A. A., \& Dennis, N. A. (2014). The cognitive control of memory: Age differences in the neural correlates of successful remembering and intentional forgetting. PLOS ONE, 9, e87010. doi: 10.1371/journal.pone.0087010

Rizio, A. A., \& Dennis, N. A. (2016). Recollection after inhibition: The effects of intentional forgetting on the neural correlates of retrieval. Cognitive Neuroscience, 8, 1-8. doi: $/ 10.1080 / 17588928.2016 .1154522$

Robinson, A. E., Hertzog, C., \& Dunlosky, J. (2006). Aging, encoding fluency, and metacognitive monitoring. Aging, Neuropsychology, and Cognition, 13, 458-478. doi: $10.1080 / 13825580600572983$

Rugg, M. D., \& Curran, T. (2007). Event-related potentials and recognition memory. Trends in Cognitive Sciences, 11, 251-257. doi: 10.1016/j.tics.2007.04.004

Russell, J. A. (1980). A circumplex model of affect. Journal of Personality and Social Psychology, 39, 1161-1178. doi: 10.1037/0022-3514.57.5.848 
Sahakyan, L., \& Foster, N. L. (2009). Intentional forgetting of actions: Comparison of listmethod and item-method directed forgetting. Journal of Memory and Language, 61, 134152. doi: 10.1016/j.jml.2009.02.006

Sahakyan, L., Waldum, E. R., Benjamin, A. S., \& Bicket, S. P. (2009). Where is the forgetting with list-method directed forgetting in recognition? Memory \& Cognition, 37, 464-476. doi:10.3758/MC.37.4.464

Sahakyan, L., \& Foster, N. L. (2015). The need for metaforgetting: Insights from directed forgetting. In J. Dunlosky \& S. Tauber (Eds.), Oxford handbook of metamemory (pp. 341-355). New York, NY: Oxford University Press.

Salthouse, T. (1996). The processing-speed theory of adult age differences in cognition. Psychological Review, 3, 403-428. doi: 10.1037/0033-295x.103.3.403

Schacter, D. L. (1999). The seven sins of memory: Insights from psychology and cognitive neuroscience. American Psychologist, 54, 182-203. doi: 10.1037/0003-066X.54.3.182

Scheibe, S., \& Carstensen, L. L. (2010). Emotional aging: Recent findings and future trends. Journal of Gerontology: Psychological Sciences, 65B, 135-144. doi: $10.1093 /$ geronb/gbp132

Schupp, H. T., Cuthbert, B. N., Bradley, M. M., Cacioppo, J. T., Ito, T., \& Lang, P. J. (2000). Affective picture processing: The late positive potential is modulated by motivational relevance. Psychophysiology, 37, 257-261. doi: 10.1111/1469-8986.3720257

Sego, S. A., Golding, J. M., \& Gottlob, L. R. (2006). Directed forgetting in older adults: Using the item and list methods. Aging, Neuropsychology, and Cognition, 13, 95-114. doi: $10.1080 / 138255890968682$ 
Sharot, T., \& Phelps, E. A. (2004). How arousal modulates memory: Disentangling the effects of attention and retention. Cognitive, Affective, \& Behavioral Neuroscience, 4, 294-306. doi: 10.3758/CABN.4.3.294

Sharot, T., \& Yonelinas, A. P. (2008). Differential time-dependent effects of emotion on recollective experience and memory for contextual information. Cognition, 106, 538-547. doi: 10.1016/j.cognition.2007.03.002

Sheard, E. D., \& MacLeod, C. M. (2005). List method directed forgetting: Return of the selective rehearsal account. In N. Ohta, C. M. MacLeod, \& B. Uttl (Eds.), Dynamic cognitive processes (pp. 219-248). Tokyo: Springer-Verlag.

Shipley, W. C. (1946). Shipley Institute of Living Scale. Los Angeles: Western Psychological Services.

Spaniol, J., \& Bayen, U. J. (2002). When is schematic knowledge used in source monitoring? Journal of Experimental Psychology: Learning, Memory, and Cognition, 28, 631-651. doi: 10.1037//0278-7393.28.4.631

Spaniol, J., Davidson, P. S., Kim, A. S., Han, H., Moscovitch, M., \& Grady, C. L. (2009). Eventrelated fMRI studies of episodic encoding and retrieval: Meta-analyses using activation likelihood estimation. Neuropsychologia, 47, 1765-1779. doi:

10.1016/j.neuropsychologia.2009.02.028

Spaniol, J. (2015). Item and source memory. In S. K. Whitbourne (Ed.), The encyclopedia of adulthood and aging (pp. 1-5). Sussex, UK: Wiley-Blackwell.

Spencer, W. D., \& Raz, N. (1995). Differential effects of aging on memory for content and context: A meta-analysis. Psychology and Aging, 10, 527-539. doi: 10.1037/08827974.10.4.527 
Stern, Y. (2002). What is cognitive reserve? Theory and research application of the reserve concept. Journal of the International Neuropsychological Society, 8, 448-460. doi: $10.1017 / \mathrm{S} 1355617702813248$

Talmi, D., \& Moscovitch, M. (2004). Can semantic relatedness explain the enhancement of memory for emotional words? Memory \& Cognition, 32, 742-751. doi: 10.3758/BF03195864

Talmi, D., Luk, B. T. C., McGarry, L. M., \& Moscovitch, M. (2007). The contribution of relatedness and distinctiveness to emotionally-enhanced memory. Journal of Memory and Language, 56, 555-574. doi: 10.1016/j.jm1.2007.01.002

Tauber, S. K., \& Dunlosky, J. (2012). Can older adults judge their learning of emotional information? Psychology and Aging, 27, 924-933. doi: 10.1037/a0028447

Thompson, K. M., Fawcett, J. M., \& Taylor, T. L. (2011). Tag, you're it: Tagging as an alternative to yes/no recognition in item method directed forgetting. Acta Psychologica, 138, 171-175. doi: 10.1016/j.actpsy.2011.06.001

Titz, C., \& Verhaeghen, P. (2010). Aging and directed forgetting in episodic memory: A metaanalysis. Psychology and Aging, 25(2), 405-411. doi: 10.1037/a0017225

Tulving, E. (1985). Memory and consciousness. Canadian Psychology, 26, 1-12. doi: $10.1037 / \mathrm{h} 0080017$

van Hooff, J. C., \& Ford, R. M. (2011). Remember to forget: ERP evidence for inhibition in an item-method directed forgetting paradigm. Brain Research, 1392, 80-92. doi: 10.1016/j.brainres.2011.04.004 
Watson, D., Clark, L. A., \& Tellegan, A. (1988). Development and validation of brief measures of positive and negative affect: The PANAS scales. Journal of Personality and Social Psychology, 54, 1063. doi: 10.1037/0022-3514.54.6.1063

Wechsler, D. (1981). Manual for the Wechsler Adult Intelligence Scale-Revised. New York, NY: Psychological Corporation.

Wilding, E. L., \& Ranganath, C. (2012). Electrophysiological correlates of episodic memory processes. In S. Kappenman \& S. J. Luck (Eds.), The oxford handbook of event-related potential components (pp. 373-396). New York, NY: Oxford University Press.

Wood, S., \& Kisley, M.A. (2006). The negativity bias is eliminated in older adults: Age-related reduction in event-related brain potentials associated with evaluative categorization. Psychology and Aging, 21, 815-820. doi: 10.1037/0882-7974.21.4.815

Wylie, G. R., Foxe, J. J., \& Taylor, T. L. (2008). Forgetting as an active process: An fMRI investigation of item-method directed forgetting. Cerebral Cortex, 18, 670-682. doi: 10.1093/cercor/bhm101

Yang, T., Lei, X., \& Anderson, M. (2016). Decreased inhibitory control of negative information in directed forgetting. International Journal of Psychophysiology, 100, 44-51. doi: 10.1016/j.ijpsycho.2015.09.007

Yang, W., Liu, P., Xiao, X., Li, X., Zeng, C., Qiu, J., \& Zhang, Q. (2012). Different neural substrates underlying directed forgetting for negative and neutral images: An eventrelated potential study. Brain Research, 1441, 53-63. doi: 10.1016/j.brainres.2011.10.042

Yang, W., Liu, P., Cui, Q., Wei, D., Li, W., Qiu, J., \& Zhang, Q. (2013) Directed forgetting of negative self-referential information is difficult: An fMRI study. PLoS ONE, e 75190. doi: 10.1371/journal.pone.0075190 
Zacks, R. T., Radvansky, G., \& Hasher, L. (1996). Studies of directed forgetting in older adults. Journal of Experimental Psychology, 22, 143-156. doi: 10.1037/0278-7393.22.1.1

Zhang, D., Xie, H., Liu, Y., \& Luo, Y. (2016). Neural correlates underlying impaired memory facilitation and suppression of negative material in depression. Scientific Reports, 6, 1-8. doi: $10.1038 / \operatorname{srep} 37556$ 